\title{
Theory of Superconductivity*
}

\author{
J. Bardeen, L. N. Cooper, $\dagger$ ANd J. R. SchriefFer $\ddagger$ \\ Department of Physics, University of Illinois, Urbana, Illinois
}

(Received July 8, 1957)

\begin{abstract}
A theory of superconductivity is presented, based on the fact that the interaction between electrons resulting from virtual exchange of phonons is attractive when the energy difference between the electrons states involved is less than the phonon energy, $\hbar \omega$. It is favorable to form a superconducting phase when this attractive interaction dominates the repulsive screened Coulomb interaction. The normal phase is described by the Bloch individual-particle model. The ground state of a superconductor, formed from a linear combination of normal state configurations in which electrons are virtually excited in pairs of opposite spin and momentum, is lower in energy than the normal state by amount proportional to an average $(\hbar \omega)^{2}$, consistent with the isotope effect. A mutually orthogonal set of excited states in
\end{abstract}

one-to-one correspondence with those of the normal phase is obtained by specifying occupation of certain Bloch states and by using the rest to form a linear combination of virtual pair configurations. The theory yields a second-order phase transition and a Meissner effect in the form suggested by Pippard. Calculated values of specific heats and penetration depths and their temperature variation are in good agreement with experiment. There is an energy gap for individual-particle excitations which decreases from about $3.5 k T_{c}$ at $T=0^{\circ} \mathrm{K}$ to zero at $T_{c}$. Tables of matrix elements of single-particle operators between the excited-state superconducting wave functions, useful for perturbation expansions and calculations of transition probabilities, are given.

\section{INTRODUCTION}

$\mathrm{T}$ HE main facts which a theory of superconductivity must explain are (1) a second-order phase transition at the critical temperature, $T_{c}$, (2) an electronic specific heat varying as $\exp \left(-T_{0} / T\right)$ near $T=0^{\circ} \mathrm{K}$ and other evidence for an energy gap for individual particle-like excitations, (3) the MeissnerOchsenfeld effect $(\mathbf{B}=0)$, (4) effects associated with infinite conductivity $(\mathbf{E}=0)$, and (5) the dependence of $T_{c}$ on isotopic mass, $T_{c} \sqrt{ } M=$ const. We present here a theory which accounts for all of these, and in addition gives good quantitative agreement for specific heats and penetration depths and their variation with temperature when evaluated from experimentally determined parameters of the theory.

When superconductivity was discovered by Onnes ${ }^{1}$ (1911), and for many years afterwards, it was thought to consist simply of a vanishing of all electrical resistance below the transition temperature. A major advance was the discovery of the Meissner effect ${ }^{2}$ (1933), which showed that a superconductor is a perfect diamagnet; magnetic flux is excluded from all but a thin penetration region near the surface. Not very long afterwards (1935), London and London ${ }^{3}$ proposed a phenomenological theory of the electromagnetic properties in which the diamagnetic aspects were assumed

* This work was supported in part by the Office of Ordnance Research, U. S. Army. One of the authors (J. R. Schrieffer) was aided by a Fellowship from the Corning Glass Works Foundation. Parts of the paper are based on a thesis submitted by Dr. Schrieffer in partial fulfillment of the requirements for a Ph.D. degree in Physics, University of Illinois, 1957.

$\dagger$ Present address: Department of Physics and Astronomy, The Ohio State University, Columbus, Ohio.

$\ddagger$ Present address: Department of Theoretical Physics, University of Birmingham, Birmingham, England.

1 H. K. Onnes, Comm. Phys. Lab. Univ. Leiden, Nos. 119, 120, 122 (1911).

${ }^{2}$ W. Meissner and R. Ochsenfeld, Naturwiss. 21, 787 (1933).

${ }^{3} \mathrm{H}$. London and F. London, Proc. Roy. Soc. (London) A149, 71 (1935); Physica 2, 341 (1935). basic. F. London ${ }^{4}$ suggested a quantum-theoretic approach to a theory in which it was assumed that there is somehow a coherence or rigidity in the superconducting state such that the wave functions are not modified very much when a magnetic field is applied. The concept of coherence has been emphasized by Pippard, ${ }^{5}$ who, on the basis of experiments on penetration phenomena, proposed a nonlocal modification of the London equations in which a coherence distance, $\xi_{0}$, is introduced. One of the authors ${ }^{6,7}$ pointed out that an energy-gap model would most likely lead to the Pippard version, and we have found this to be true of the present theory. Our theory of the diamagnetic aspects thus follows along the general lines suggested by London and by Pippard. ${ }^{7}$

The Sommerfeld-Bloch individual-particle model (1928) gives a fairly good description of normal metals, but fails to account for superconductivity. In this theory, it is assumed that in first approximation one may neglect correlations between the positions of the electrons and assume that each electron moves independently in some sort of self-consistent field determined by the other conduction electrons and the ions. Wave functions of the metal as a whole are designated by occupation of Bloch individual-particle states of energy $\epsilon(\mathbf{k})$ defined by wave vector $\mathbf{k}$ and spin $\sigma$; in the ground state all levels with energies below the Fermi energy, $\mathcal{E}_{F}$, are occupied; those above are unoccupied. Left out of the Bloch model are correlations between electrons brought about by Coulomb forces and interactions between electrons and lattice vibrations (or phonons).

${ }^{4}$ F. London, Proc. Roy. Soc. (London) A152, 24 (1935); Phys. Rev. 74, 562 (1948).

5 A. B. Pippard, Proc. Roy. Soc. (London) A216, 547 (1953).

6 J. Bardeen, Phys. Rev. 97, 1724 (1955).

7 For a recent review of the theory of superconductivity, which includes a discussion of the diamagnetic properties, see J. Bardeen, Encyclopedia of Physics (Springer-Verlag, Berlin, 1956), Vol. 15, p. 274 . 
Most of the relatively large energy associated with correlation effects occurs in both normal and superconducting phases and cancels out in the difference. One of the problems in constructing a satisfactory microscopic theory of superconductivity has been to isolate that part of the interaction which is responsible for the transition. Heisenberg ${ }^{8}$ and $\mathrm{Koppe}^{9}$ proposed a theory based on long-wavelength components of the Coulomb interaction, which were presumed to give fluctuations in electron density described roughly by wave packets localizing a small fraction of the electrons on lattices moving in different directions. A great break-through occurred with the discovery of the isotope effect, ${ }^{10}$ which strongly indicated, as had been suggested independently by Fröhlich, ${ }^{11}$ that electronphonon interactions are primarily responsible for superconductivity.

Early theories based on electron-phonon interactions have not been successful. Fröhlich's theory, which makes use of a perturbation-theoretic approach, does give the correct isotopic mass dependence for $H_{0}$, the critical field at $T=0^{\circ} \mathrm{K}$, but does not yield a phase with superconducting properties and further, the energy difference between what is supposed to correspond to normal and superconducting phases is far too large. A variational approach by one of the authors ${ }^{12}$ ran into similar difficulties. Both theories are based primarily on the self-energy of the electrons in the phonon field rather than on the true interaction between electrons, although it was recognized that the latter might be important. ${ }^{13}$

The electron-phonon interaction gives a scattering from a Bloch state defined by the wave vector $\mathbf{k}$ to $\mathbf{k}^{\prime}=\mathbf{k} \pm \boldsymbol{k}$ by absorption or emission of a phonon of wave vector $\boldsymbol{\kappa}$. It is this interaction which is responsible for thermal scattering. Its contribution to the energy can be estimated by making a canonical transformation which eliminates the linear electron-phonon interaction terms from the Hamiltonian. In second order, there is one term which gives a renormalization of the phonon frequencies, and another, $\mathrm{H}_{2}$, which gives a true interaction between electrons, independent of the vibrational amplitudes. A transformation of this sort was given first by Fröhlich ${ }^{14}$ in a formulation in which Coulomb interactions between electrons were disregarded. In a later treatment, Nakajima ${ }^{15}$ showed how such inter-

\footnotetext{
${ }^{8}$ W. Heisenberg, Two Lectures (Cambridge University Press, Cambridge, 1948).

${ }^{9}$ H. Koppe, Ergeb. exakt. Naturw. 23, 283 (1950); Z. Physik 148, 135 (1957)

${ }^{10}$ E. Maxwell, Phys. Rev. 78, 477 (1950); Reynolds, Serin, Wright, and Nesbitt, Phys. Rev. 78, 487 (1950).

${ }^{11}$ H. Fröhlich, Phys. Rev. 79, 845 (1950).

12 J. Bardeen, Phys. Rev. 79, 167 (1950); 80, 567 (1950); 81, 829 (1951).

${ }^{13}$ For a review of the early work, see J. Bardeen, Revs. Modern

Phys. 23, 261 (1951).

${ }_{14}$ H. Fröhlich, Proc. Roy. Soc. (London) A215, 291 (1952).

${ }^{15} \mathrm{~S}$. Nakajima, Proceedings of the International Conference on Theoretical Physics, Kyoto and Tokyo, September, 1953 (Science
} Council of Japan, Tokyo, 1954). actions could be included. Particularly for the longwavelength part of the interaction, it is important to take into account the screening of the Coulomb field of any one electron by other conduction electrons. Such effects are included in a more complete analysis by Bardeen and Pines, ${ }^{16}$ based on the Bohm-Pines collective model, in which plasma modes are introduced for long wavelengths.

We shall call the interaction, $H_{2}$, between electrons resulting from the electron-phonon interaction the "phonon interaction." This interaction is attractive when the energy difference, $\Delta \epsilon$, between the electron states involved is less than $\hbar \omega$. Diagonal or self-energy terms of $H_{2}$ give an energy of order of $-N\left(\mathcal{E}_{F}\right)(\hbar \omega)^{2}$, where $N\left(\mathcal{E}_{F}\right)$ is the density of states per unit energy at the Fermi surface. The theories of Fröhlich and Bardeen mentioned above were based largely on this part of the energy. The observed energy differences between superconducting and normal states at $T=0^{\circ} \mathrm{K}$ are much smaller, of the order of $-N\left(\mathcal{E}_{F}\right)\left(k T_{c}\right)^{2}$ or about $10^{-8}$ ev/atom. The present theory, based on the off-diagonal elements of $\mathrm{H}_{2}$ and the screened Coulomb interaction, gives energies of the correct order of magnitude. While the self-energy terms do depend to some extent on the distribution of electrons in $\mathbf{k}$ space, it is now believed that this part of the energy is substantially the same in the normal and superconducting phases. The self-energy terms are also nearly the same for all of the various excited normal state configurations which make up the superconducting wave functions.

In a preliminary communication, ${ }^{17}$ we gave as a criterion for the occurrence of a superconducting phase that for transitions such that $\Delta \epsilon<\hbar \omega$, the attractive $\mathrm{H}_{2}$ dominate the repulsive short-range screened Coulomb interaction between electrons, so as to give a net attraction. We showed how an attractive interaction of this sort can give rise to a cooperative many-particle state which is lower in energy than the normal state by an amount proportional to $(\hbar \omega)^{2}$, consistent with the isotope effect. We have since extended the theory to higher temperatures, have shown that it gives both a second-order transition and a Meissner effect, and have calculated specific heats and penetration depths.

In the theory, the normal state is described by the Bloch individual-particle model. The ground-state wave function of a superconductor is formed by taking a linear combination of many low-lying normal state configurations in which the Bloch states are virtually occupied in pairs of opposite spin and momentum. If the state $\mathbf{k} \uparrow$ is occupied in any configuration, $-\mathbf{k} \downarrow$ is also occupied. The average excitation energy of the virtual pairs above the Fermi sea is of the order of $k T_{c}$. Excited states of the superconductor are formed by specifying occupation of certain Bloch states and by using all of the rest to form a linear combination of

${ }_{16}^{16}$ J. Bardeen and D. Pines, Phys. Rev. 99, 1140 (1955). 17 Bardeen, Cooper, and Schrieffer, Phys. Rev. 106, 162 (1957). 
virtual pair configurations. There is thus a one-to-one correspondence between excited states of the normal and superconducting phases. The theory yields an energy gap for excitation of individual electrons from the superconducting ground state of about the observed order of magnitude.

The most important concribution to the interaction energy is given by short- rather than long-wavelength phonons. Our wave functions for the suerconducting phase give a coherence of short-wavelength components of the density matrix which extend over large distances in real space, so as to take maximum advantage of the attractive part of the interaction. The coherence distance, of the order of Pippard's $\xi_{0}$, can be estimated from uncertainty principle arguments..$^{5,7}$ If intervals of the order of $\Delta k \sim\left(k T_{c} / \mathcal{E}_{F}\right) k_{F} \sim 10^{4} \mathrm{~cm}^{-1}$ are important in $\mathbf{k}$ space, wave functions in real space must extend over distances of at least $\Delta x \sim 1 / \Delta k \sim 10^{-4} \mathrm{~cm}$. The fraction of the total number of electrons which have energies within $k T_{c}$ of the Fermi surface, so that they can interact effectively, is approximately $k T_{c} / \mathcal{E}_{F} \sim 10^{-4}$. The number of these in an interaction region of volume $(\Delta x)^{3}$ is of the order of $10^{22} \times\left(10^{-4}\right)^{3} \times 10^{-4}=10^{6}$. Thus our wave functions must describe coherence of large numbers of electrons. ${ }^{18}$

In the absence of a satisfactory microscopic theory, there has been considerable development of phenomenological theories for both thermal and electromagnetic properties. Of the various two-fluid models used to describe the thermal properties, the first and best known is that of Gorter and Casimir, ${ }^{19}$ which yields a parabolic critical field curve and an electronic specific heat varying as $T^{3}$. In this, as well as in subsequent theories of thermal properties, it is assumed that all of the entropy of the electrons comes from excitations of individual particles from the ground state. In recent years, there has been considerable experimental evidence $^{20}$ for an energy gap for such excitations, decreasing from $\sim 3 k T_{c}$ at $T=0^{\circ} \mathrm{K}$ to zero at $T=T_{c}$. Two-fluid models which yield an energy gap and an exponential specific heat curve at low temperatures have been discussed by Ginsburg ${ }^{21}$ and by Bernardes. ${ }^{22}$ Koppe's

\footnotetext{
${ }^{18}$ Our picture differs from that of Schafroth, Butler, and Blatt, Helv. Phys. Acta 30, 93 (1957), who suggest that pseudomolecules of pairs of electrons of opposite spin are formed. They show if the size of the pseudomolecules is less than the average distance between them, and if other conditions are fulfilled, the system has properties similar to that of a charged Bose-Einstein gas, including a Meissner effect and a critical temperature of condensation. Our pairs are not localized in this sense, and our transition is not analogous to a Bose-Einstein condensation.

${ }^{19}$ C. J. Gorter and H. B. G. Casimir, Physik. Z. 35, 963 (1934); Z. techn. Physik 15, 539 (1934).

${ }^{20}$ For discussions of evidence for an energy gap, see Blevins, Gordy, and Fairbank, Phys. Rev. 100, 1215 (1955); Corak, Goodman, Satterthwaite, and Wexler, Phys. Rev. 102, 656 (1956) W. S. Corak and C. B. Satterthwaite, Phys. Rev. 102, 662 (1956) R. E. Glover and M. Tinkham, Phys. Rev. 104, 844 (1956), and to be published.

${ }^{21}$ W. L. Ginsburg, Fortschr. Physik 1, 101 (1953); also see reference 7.

${ }^{22}$ N. Bernardes, Phys. Rev. 107, 354 (1957).
}

theory may also be interpreted in terms of an energygap model. ${ }^{7}$ Our theory yields an energy gap and specific heat curve consistent with the experimental observations.

The best known of the phenomenological theories for the electromagnetic properties is that of $\mathrm{F}$. and $\mathrm{H}$. London..$^{23}$ With an appropriate choice of gauge for the vector potential, $\mathbf{A}$, the London equation for the superconducting current density, $\mathbf{j}$, may be written

$$
-c \Lambda \mathbf{j}=\mathbf{A} \text {. }
$$

The London penetration depth is given by:

$$
\lambda_{L}{ }^{2}=\Lambda c^{2} / 4 \pi \text {. }
$$

F. London has pointed out that (1.1) would follow from quantum theory if the superconducting wave functions are so rigid that they are not modified at all by the application of a magnetic field. For an electron density $n / \mathrm{cm}^{3}$, this approach gives $\Lambda=m / n e^{2}$.

On the basis of empirical evidence, Pippard ${ }^{5}$ has proposed a modification of the London equation in which the current density at a point is given by an integral of the vector potential over a region surrounding the point:

$$
\mathbf{j}(\mathbf{r})=-\frac{3}{4 \pi c \Lambda \xi_{0}} \int \frac{\mathbf{R}\left[\mathbf{R} \cdot \mathbf{A}\left(\mathbf{r}^{\prime}\right)\right] e^{-R / \xi_{0}}}{R^{4}} d \tau^{\prime}
$$

where $\mathbf{R}=\mathbf{r}-\mathbf{r}^{\prime}$. The "coherence distance," $\xi_{0}$, is of the order of $10^{-4} \mathrm{~cm}$ in a pure metal. For a very slowly varying $\mathbf{A}$, the Pippard expression reduces to the London form (1.1).

The present theory indicates that the Meissner effect is intimately related to the existence of an energy gap, and we are led to a theory similar to, although not quite the same as, that proposed by Pippard. Our theoretical values for $\xi_{0}$ are close to those derived empirically by Pippard. We find that while the integrand is relatively independent of temperature, the coefficient in front of the integral (in effect $\Lambda$ ) varies with $T$ in such a way as to account for the temperature variation of penetration depth.

Our theory also accounts in a qualitative way for those aspects of superconductivity associated with infinite conductivity and a persistent current flowing in a ring. When there is a net current flow, the paired states $\left(\mathbf{k}_{1} \uparrow, \mathbf{k}_{2} \downarrow\right)$ have a net momentum $\mathbf{k}_{1}+\mathbf{k}_{2}=\mathbf{q}$, where $\mathbf{q}$ is the same for all virtual pairs. For each value of $\mathbf{q}$, there is a metastable state with a minimum in free energy and a unique current density. Scattering of individual electrons will not change the value of $\mathbf{q}$ common to virtual pair states, and so can only produce fluctuations about the current determined by q. Nearly all fluctuations will increase the free energy; only those which involve a majority of the electrons so as to change

${ }^{23}$ An excellent account may be found in F. London, Superfluids (John Wiley and Sons, Inc., New York, 1954), Vol. 1. 
the common $\mathbf{q}$ can decrease the free energy. These latter are presumably extremely rare, so that the metastable current carrying state can persist indefinitely. ${ }^{24}$

It has long been recognized that there is a law of corresponding states for superconductors. The various properties can be expressed approximatly in terms of a small number of parameters. If the ratio of the electronic specific heat at $T$ to that of the normal state at $T_{c}, C_{s}(T) / C_{n}\left(T_{c}\right)$, is plotted on a reduced temperature scale, $t=T / T_{c}$, most superconductors fall on nearly the same curve. There are two parameters involved: (1) the density of states in energy at the Fermi surface, $N\left(\mathscr{E}_{F}\right)$, determined from $C_{n}(T)=\gamma T$ and (2) one which depends on the phonon interaction, which can be estimated from $T_{c}$. A consequence of the similarity law is that $\gamma T_{c}^{2} / V_{m} H_{0}^{2}$ (where $V_{m}$ is the molar volume and $H_{0}$ the critical field at $T=0^{\circ} \mathrm{K}$ ) is approximately the same for most superconductors.

A third parameter, the average velocity, $v_{0}$, of electrons at the Fermi surface,

$$
v_{0}=\hbar^{-1}|\partial \mathcal{E} / \partial \mathbf{k}|_{F}
$$

is required for penetration phenomena. As pointed out by Faber and Pippard, ${ }^{25}$ this parameter is most conveniently determined from measurements of the anomalous skin effect in normal metals in the high-frequency limit. The expression, as given by Chambers $^{26}$ for the current density when the electric field varies over a mean free path, $l$, may be written in the form:

$$
\mathbf{j}_{n}(\boldsymbol{r})=\frac{e^{2} N\left(\mathcal{E}_{F}\right) v_{0}}{2 \pi} \int \frac{\mathbf{R}\left[\mathbf{R} \cdot \mathbf{A}\left(\mathbf{r}^{\prime}\right)\right] e^{-R / l}}{R^{4}} d \tau^{\prime} .
$$

The coefficient $N\left(\mathcal{E}_{F}\right) v_{0}$ has been determined empirically for tin and aluminum.

Pippard based his Eq. (1.3) on Chambers' expression. London's coefficient, $\Lambda$, for $T=0^{\circ} \mathrm{K}$ may be expressed in the form:

$$
\Lambda^{-1}=\frac{2}{3} e^{2} N\left(\mathcal{E}_{F}\right) v_{0}^{2} .
$$

Faber and Pippard suggest that if $\xi_{0}$ is written:

$$
\xi_{0}=a \hbar v_{0} / k T_{c}
$$

the dimensionless constant $a$ has approximately the

\footnotetext{
${ }^{24}$ Blatt, Butler, and Schafroth, Phys. Rev. 100, 481 (1955) have introduced the concept of a "correlation length," roughly the distance over which the momenta of a pair of particles are correlated. M. R. Schafroth, Phys. Rev. 100, 502 (1955), has argued that there is a true Meissner effect only if the correlation length is effectively infinite. In our theory, the correlation length (not to be confused with Pippard's coherence distance, $\xi_{0}$ ) is most reasonably interpreted as the distance over which the momentum of virtual pairs is the same. We believe that in this sense, the correlation length is effectively infinite. The value of $\mathbf{q}$ is exactly zero everywhere in a simply connected body in an external field. When there is current flow, as in a torus, there is a unique distribution of $\mathbf{q}$ values for minimum free energy.

${ }^{25}$ T. E. Faber and A. B. Pippard, Proc. Roy. Soc. (London) A231, 53 (1955).

${ }^{26}$ See A. P. Pippard, Advances in Electronics (Academic Press, Inc., New York, 1954), Vol. 6, p. 1.
}

same value for all superconductors and they find it equal to about 0.15 for $\mathrm{Sn}$ and $\mathrm{Al}^{27}$

Our theory is based on a rather idealized model in which anisotropic effects are neglected. It contains three parameters, two corresponding to $N\left(\mathcal{E}_{F}\right)$ and $v_{0}$, and one dependent on the electron-phonon interaction which determines $T_{c}$. The model appears to fit the law of corresponding states about as well as real metals do ( $\sim 10 \%$ for most properties). We find a relation corresponding to (1.7) with $a=0.18$. It thus appears that superconducting properties are not dependent on the details of the band structure but only upon the gross features.

Section II is concerned with the nature of the ground state and the energy of excited states near $T=0^{\circ} \mathrm{K}$, Sec. III with excited states and thermal properties, Sec. IV with calculation of matrix elements for application to perturbation theory expansions and transition probabilities and Sec. V with electrodynamic and penetration phenomenon. Some of the computational details are given in Appendices.

We give a fairly complete account of the equilibrium properties of our model, but nothing on transport or boundary effects. Starting from matrix elements of single-particle scattering operators as given in Sec. IV, it should not be difficult to determine transport properties in the superconducting state from the corresponding properties of the normal state.

\section{THE GROUND STATE}

The interaction which produces the energy difference between the normal and superconducting phases in our theory arises from the virtual exchange of phonons and the screened Coulomb repulsion between electrons. Other interactions, such as those giving rise to the single-particle self-energies, are thought to be essentially the same in both states, their effects thus cancelling in the energy difference. The problem is therefore one of calculating the ground state and excited states of a dense system of fermions interacting via two-body potentials.

The Hamiltonian for the fermion system is most conveniently expressed in terms of creation and annihilation operators, based on the renormalized Bloch states specified by wave vector $\mathbf{k}$ and $\operatorname{spin} \sigma$, which satisfy the usual Fermi commutation relations:

$$
\begin{aligned}
{\left[c_{\mathrm{k} \sigma}, c_{\mathrm{k}^{\prime} \sigma^{\prime}} *\right]_{+} } & =\delta_{\mathrm{kk}^{\prime}} \delta_{\sigma \sigma^{\prime}}, \\
{\left[c_{\mathrm{k} \sigma}, c_{\mathrm{k}^{\prime} \sigma^{\prime}}\right]_{+} } & =0 .
\end{aligned}
$$

The single-particle number operator $n_{\mathrm{k} \sigma}$ is defined as

$$
n_{\mathrm{k} \sigma}=c_{\mathrm{k} \sigma}{ }^{*} c_{\mathrm{k} \sigma} .
$$

The Hamiltonian for the electrons may be expressed in

${ }^{27}$ From analysis of data on transmission of microwave and far infrared radiation through superconducting films of tin and lead, Glover and Tinkham (reference 20) find $a=0.27$. 
the form

$$
\begin{array}{r}
H=\sum_{k>k_{F}} \epsilon_{\mathrm{k}} n_{\mathbf{k} \sigma}+\sum_{k<k_{F}}\left|\epsilon_{\mathrm{k}}\right|\left(1-n_{\mathrm{k} \sigma}\right)+H_{\mathrm{Coul}}+\frac{1}{2} \sum_{\mathbf{k}, \mathbf{k}^{\prime}, \sigma, \sigma^{\prime}, \mathbf{k}} \\
\times \frac{2 \hbar \omega_{\kappa}\left|M_{\boldsymbol{k}}\right|^{2} c^{*}\left(\mathbf{k}^{\prime}-\mathbf{k}, \sigma^{\prime}\right) c\left(\mathbf{k}^{\prime}, \sigma^{\prime}\right) c^{*}(\mathbf{k}+\boldsymbol{k}, \sigma) c(\mathbf{k}, \sigma)}{\left(\epsilon_{\mathrm{k}}-\epsilon_{\mathrm{k}+\kappa}\right)^{2}-\left(\hbar \omega_{k}\right)^{2}} \\
=H_{0}+H_{I},
\end{array}
$$

where $\epsilon_{\mathrm{k}}$ is the Bloch energy measured relative to the Fermi energy, $\mathcal{E}_{F}$. We denote by $k>k_{F}$ states above the Fermi surface, by $k<k_{F}$ those below. The fourth term on the right of (2.4) is $H_{2}$, the phonon interaction, which comes from virtual exchange of phonons between the electrons. The matrix element for phonon-electron interaction, $M_{\kappa}$, calculated for the zero-point amplitude of the lattice vibrations, is related to the $v_{\kappa}$ introduced by Bardeen and Pines ${ }^{16}$ by

$$
\left|M_{\kappa}\right|^{2}=\left|v_{\kappa}\right|^{2}\left\langle q_{\kappa}^{2}\right\rangle_{\mathrm{Av}}=\left|v_{\kappa}\right|^{2}\left(\hbar / 2 \omega_{\kappa}\right) .
$$

Since $\left|M_{\kappa}\right|^{2}$ varies with isotopic mass in the same way that $\omega_{\kappa}$ does, the ratio $\left|M_{\kappa}\right|^{2} / \hbar \omega_{\kappa}$ is independent of isotopic mass. We consider only the off-diagonal interaction terms of $H_{2}$, assuming that the diagonal terms are taken into account by appropriate renormalization of the Bloch energies, $\epsilon_{\mathrm{k}}$. The third term is the screened Coulomb interaction.

Following Bardeen and Pines, ${ }^{16}$ the phonons are assumed to be decoupled from the electrons by a renormalization procedure and their frequencies are taken to be unaltered by the transition to the superconducting state. While this assumption is not strictly valid, the shift in self-energy can be taken into account after we have solved for the electronic part of the wave function. This separation is possible because the phonons depend only upon the average electron distribution in momentum space and the wave function for electrons at any temperature is formed from configurations with essentially the same distribution of particles. The Bloch energies are also assumed to be constant; however, their shift with temperature could be treated as in the phonon case.

The form of the phonon interaction shows that it is attractive (negative) for excitation energies $\left|\epsilon_{\mathrm{k}}-\epsilon_{\mathrm{k}+\kappa}\right|$ $<\hbar \omega_{k}$. Opposed to this is the repulsive Coulomb interaction, which may be expressed in a form similar to $\mathrm{H}_{2}$. For free electrons in a system of unit volume the interaction in momentum space is $4 \pi e^{2} / \kappa^{2}$. In the BohmPines theory, the long-wavelength components are expressed in the form of plasma oscillations, so that $\kappa$ can be no smaller than minimum value $\kappa_{c}$, usually slightly less than the radius of the Fermi surface, $k_{F}$. One could also take screening into account by a FermiThomas method, in which case $\kappa^{2}$ would be replaced by $\kappa^{2}+\kappa_{s}^{2}$, where $\kappa_{8}$ depends on the electron density. Our criterion for superconductivity is that the attractive phonon interaction dominate the Coulomb interaction for those matrix elements which are of importance in the superconducting wave function:

$$
-V \equiv\left\langle-\frac{2\left|M_{x}\right|^{2}}{\hbar \omega_{x}}+\frac{4 \pi e^{2}}{\kappa^{2}}\right\rangle_{\text {Av }}<0 .
$$

The most important transitions are those for which $\left|\epsilon_{\mathrm{k}}-\epsilon_{\mathrm{k}+\kappa}\right| \sim k T_{c} \ll \hbar \omega_{\kappa}$. A detailed discussion of the criterion (2.6) has been given by Pines, ${ }^{28}$ who shows that it accounts in a reasonable way for the empirical rules of Matthias ${ }^{29}$ for the occurrence of superconductivity. Numerically, the criterion is not much different from one given earlier by Fröhlich, ${ }^{11}$ based on a different principle.

To obtain the ground state function, we observe that the interaction Hamiltonian connects a large number of nearly degenerate occupation number configurations with each other via nonzero matrix elements. If the matrix elements were all negative in sign, one could obtain a state with low energy by forming a linear combination of the basis functions with expansion coefficients of the same sign. The magnitude of the interaction energy obtained in this manner would be approximately given by the number of configurations which connect to a given typical configuration times an average matrix element. This was demonstrated by one of the authors ${ }^{30}$ by solving a problem in which two electrons with zero total momentum interact via constant negative matrix elements in a small shell above the Fermi surface. It was shown that the ground state of this system is separated from the continuum by a volume independent energy. This type of coherent mixing of Bloch states produces a state with qualitatively different properties from the original states.

In the actual problem, the interaction which takes a pair from $\left(\mathbf{k}_{1} \sigma_{1}, \mathbf{k}_{2} \sigma_{2}\right)$ to $\left(\mathbf{k}_{1}{ }^{\prime} \sigma_{1}, \mathbf{k}_{2}{ }^{\prime} \sigma_{2}\right)$ contains the operators,

$$
c^{*}\left(\mathbf{k}_{2}{ }^{\prime}, \sigma_{2}\right) c\left(\mathbf{k}_{2}, \sigma_{2}\right) c^{*}\left(\mathbf{k}_{1}{ }^{\prime}, \sigma_{1}\right) c\left(\mathbf{k}_{1}, \sigma_{1}\right) .
$$

Conservation of momentum requires that

$$
\mathbf{k}_{1}+\mathbf{k}_{2}=\mathbf{k}_{1}{ }^{\prime}+\mathbf{k}_{2}{ }^{\prime} \text {. }
$$

Because of Fermi-Dirac statistics, matrix elements of (2.7) between arbitrary many electron configurations alternate in sign so that if the configurations occur in the ground state with roughly equal weight, the net interaction energy would be small. We can, however, produce a coherent low state by choosing a subset of configurations between which the matrix elements are negative. Such a subset can be formed by those configurations in which the Bloch states are occupied in pairs, $\left(\mathbf{k}_{1} \sigma_{1}, \mathbf{k}_{2} \sigma_{2}\right)$; that is, if one member of the pair is occupied in any configuration in the subset, the other is also. Since the interaction conserves momentum, a

\footnotetext{
${ }^{28} \mathrm{D}$. Pines (to be published).

${ }^{29}$ B. Matthias, Progress in Low Temperature Physics (NorthHolland Publishing Company, Amsterdam, 1957), Vol. 2.

${ }^{30}$ L. N. Cooper, Phys. Rev. 104, 1189 (1956).
} 
maximum number of matrix elements will be obtained if all pairs have the same net momentum, $\mathbf{k}_{1}+\mathbf{k}_{2}=\mathbf{q}$. It is further desirable to take pairs of opposite spin, because exchange terms reduce the interaction for parallel spins. The best choice for $q$ for the ground state pairing is $\mathbf{q}=0,(\mathbf{k} \uparrow,-\mathbf{k} \downarrow)$.

We start then by considering a reduced problem in which we include only configurations in which the states are occupied in pairs such that if $\mathbf{k} \uparrow$ is occupied so is $-\mathbf{k} \downarrow$. A pair is designated by the wave vector $\mathbf{k}$, independent of spin. Creation and annihilation operators for pairs may be defined in terms of the singleparticle operators as follows:

$$
\begin{gathered}
b_{\mathrm{k}}=c_{-\mathrm{k} \downarrow} c_{\mathrm{k} \uparrow}, \\
b_{\mathrm{k}}{ }^{*}=c_{\mathrm{k}} \uparrow^{*} c_{-\mathrm{k} \downarrow} * .
\end{gathered}
$$

These operators satisfy the commutation relations

$$
\begin{aligned}
{\left[b_{\mathrm{k}}, b_{\mathrm{k}^{\prime}} *\right]_{-} } & =\left(1-n_{\mathrm{k} \uparrow}-n_{-\mathrm{k} \downarrow}\right) \delta_{\mathrm{kk}^{\prime}}, \\
{\left[b_{\mathrm{k}}, b_{\mathrm{k}^{\prime}}\right]_{-} } & =0, \\
{\left[b_{\mathrm{k}}, b_{\mathrm{k}^{\prime}}\right]_{+} } & =2 b_{\mathrm{k}} b_{\mathrm{k}^{\prime}}\left(1-\delta_{\mathrm{kk}^{\prime}}\right),
\end{aligned}
$$

where $n_{\mathrm{k} \sigma}$ is given by (2.3). While the commutation relation (2.12) is the same as for bosons, the commutators (2.11) and (2.13) are distinctly different from those for Bose particles. The factors $\left(1-n_{\mathrm{k} \uparrow}-n_{-\mathrm{k} \downarrow}\right)$ and $\left(1-\delta_{\mathrm{kk}^{\prime}}\right)$ arise from the effect of the exclusion principle on the single particles.

That part of the Hamiltonian which connects pairs with zero net momentum may be derived from the Hamiltonian (2.4) and expressed in terms of the $b$ 's. Measuring the energy relative to the Fermi sea, we obtain :

$$
\begin{aligned}
H_{\mathrm{red}}=2 \sum_{k>k_{F}} \epsilon_{\mathrm{k}} b_{\mathrm{k}}{ }^{*} b_{\mathrm{k}}+2 \sum_{k<k_{F}}\left|\epsilon_{\mathrm{k}}\right| & b_{\mathrm{k}} b_{\mathrm{k}}{ }^{*} \\
& -\sum_{\mathbf{k} \mathbf{k}^{\prime}} V_{\mathrm{kk}^{\prime}} b_{\mathbf{k}^{\prime}} * b_{\mathrm{k}} .
\end{aligned}
$$

We have defined the interaction terms with a negative sign so that $V_{\mathrm{kk}^{\prime}}$ will be predominantly positive for a superconductor. There are many other terms in the complete interaction which connect pairs with total momentum $\mathbf{q} \neq 0$. These have little effect on the energy, and can be treated as a perturbation. Although the interaction terms kept in $H_{\text {red }}$ may appear to have a negligible weight, it is this part which contributes overwhelmingly to the interaction energy.

We have used a Hartree-like method to determine the expansion coefficients, which appears to give an excellent approximation, and may, indeed, even be correct in the limit of a large number of particles. ${ }^{31}$ (See Appendix A.)

${ }^{31}$ Since (2.14) is quadratic in the $b$ 's, one might hope to get an exact solution for the ground state by an appropriate redefinition of the single-particle states, as can be done for either Fermi-Dirac or Einstein-Bose statistics. Our pairs obey neither of these, and no such simple solution appears possible.
Excited states are treated in much the same way as the ground state. One must distinguish between singly excited particles, in which one and only one of a pair $(\mathbf{k} \uparrow,-\mathbf{k} \downarrow)$ is occupied, and excited or "real" pair states. We treat singly excited particles in the Bloch scheme, as in the normal metal. They contribute a negligible amount to the interaction energy directly, but reduce the amount of phase space available for real and virtual pairs. Thus the interaction portion of $H_{\mathrm{red}}$ is modified by deleting from the sums over $\mathbf{k}$ and $\mathbf{k}^{\prime}$ all singly occupied states, and the remainder is used to determine the interaction energy associated with the pairs.

One might expect to get some interaction energy from singly occupied states by associating them in various pairs with $\mathbf{q} \neq 0$. However, an appreciable energy is obtained only if a finite fraction of the pairs have the same $\mathbf{q}$, and this will not be true for randomly excited particles. States with a net current flow can be obtained by taking a pairing $\left(\mathbf{k}_{1} \uparrow, \mathbf{k}_{2} \downarrow\right)$, with $\mathbf{k}_{1}+\mathbf{k}_{2}=\mathbf{q}$, and $\mathbf{q}$ the same for all virtual pairs.

The most general wave function satisfying the pairing condition $(\mathbf{k} \uparrow,-\mathbf{k} \downarrow)$ is of the form

$$
\sum_{\mathbf{k}_{1} \cdots \mathbf{k}_{n}}\left[h\left(\mathbf{k}_{1} \cdots \mathbf{k}_{n}\right)\right]^{\frac{1}{2}} f\left(\cdots 1\left(k_{1}\right) \cdots 1\left(k_{n}\right) \cdots\right),
$$

where the sum extends over all distinct pair configurations. To construct our ground state function we make a Hartree-like approximation in which the probability that a specific configuration of pairs occurs in the wave function is given by a product of occupancy probabilities for the individual pair states. If for the moment we relax the requirement that the wave function describes a system with a fixed number of particles, then a function having this Hartree-like property is

$$
\Psi=\prod_{\mathrm{k}}\left[\left(1-h_{\mathrm{k}}\right)^{\frac{1}{2}}+h_{\mathrm{k}}^{\frac{1}{2}} b_{\mathrm{k}}{ }^{*}\right] \Phi_{0},
$$

where $\Phi_{0}$ is the vacuum. It follows from (2.16) that the probability of the $n$ states $\mathbf{k}_{1} \cdots \mathbf{k}_{n}$ being occupied is $h\left(\mathbf{k}_{1}\right) \cdots h\left(\mathbf{k}_{n}\right)$, and since $n$ is unrestricted we see that $\Psi$ is closely related to the intermediate coupling approximation.

For any specified wave vector $\mathbf{k}^{\prime}$, it is convenient to decompose $\Psi$ into two components, in one of which, $\varphi_{1}$, the pair state designated by $\mathbf{k}^{\prime}$ is certainly occupied and the other, $\varphi_{0}$, for which it is empty:

$$
\Psi=h_{\mathrm{k}^{\prime}}{ }^{\frac{1}{2}} \varphi_{1}+\left(1-h_{\mathrm{k}^{\prime}}\right)^{\frac{1}{2}} \varphi_{0} .
$$

The coefficient $h_{\mathbf{k}^{\prime}}$ is the probability that state $\mathbf{k}^{\prime}$ is occupied and the $\varphi$ 's are the normalized functions:

$$
\varphi_{1}=b_{\mathrm{k}^{\prime}} * \varphi_{0}=b_{\mathrm{k}^{\prime}} * \prod_{\mathrm{k}\left(\neq \mathbf{k}^{\prime}\right)}\left[\left(1-h_{\mathrm{k}}\right)^{\frac{1}{2}}+h_{\mathrm{k}^{\frac{1}{2}}} b_{\mathrm{k}} *\right] \Phi_{0} .
$$

In the limit of a large system, the weights of states with different total numbers of pairs in $\Psi$ will be sharply peaked about the average number, $N$, which will be dependent on the choice of the $h$ 's. We take for our 
ground state function, $\Psi_{N}$, the projection of $\Psi$ onto the space of exactly $N$ pairs. ${ }^{32}$ This function may also be decomposed as in (2.17), but since $\varphi_{N 1}$ and $\varphi_{N 0}$ now have the same number of pairs, $\varphi_{N 1}$ is not equal to $b_{\mathrm{k}^{\prime}} * \varphi_{N 0}$. To decompose $\Psi_{N}$, we suppose that $\mathbf{k}$ space is divided into elements, $\Delta \mathbf{k}$, with $\mathfrak{T}_{\mathbf{k}}$ available states of which in a typical configuration $m_{\mathrm{k}}$ are occupied by pairs. The $m_{\mathrm{k}}$ 's are restricted so that the total number of pairs is specified:

$$
\sum_{\text {all } \Delta \mathrm{k}} m_{\mathrm{k}}=N=\sum_{\text {all } \Delta \mathrm{k}}\left\langle m_{\mathrm{k}}\right\rangle_{\mathrm{Av}}
$$

The total weight of a given distribution of $m_{\mathrm{k}}$ 's in $\Psi$ is

$$
W\left(m_{\mathrm{k}}\right)=\prod_{\text {all } \Delta \mathrm{k}} \frac{\mathfrak{N}_{k} !}{m_{\mathrm{k}} !\left(\mathscr{N}_{\mathrm{k}}-m_{k}\right) !} h_{\mathrm{k}}^{m_{k}}\left(1-h_{\mathrm{k}}\right)^{\mathfrak{N}_{\mathrm{k}-m_{\mathrm{k}}}}
$$

and the total weight of functions with specified $N$ is

$$
W_{N}=\sum_{\left(\Sigma m_{\mathrm{k}}=N\right)} W\left(m_{\mathrm{k}}\right)
$$

where the sum is over-all distributions of the $m_{\mathrm{k}}$ 's subject to the conditions (2.19).

The decomposition of $\Psi_{N}$ into a part in which a specified pair state $\mathbf{k}^{\prime}$ is occupied and one where it is not can be carried out by calculating from $\Psi_{N}$ the total weight, $W_{N, \mathrm{k}^{\prime}}$, corresponding to $\mathbf{k}^{\prime}$ occupied with the restriction $\sum m_{\mathrm{k}}=N$. When $\mathbf{k}^{\prime}$ is occupied, there are $\mathscr{K}_{\mathrm{k}^{\prime}}-1$ other states in the cell over which the remaining $m_{\mathrm{k}^{\prime}}-1$ particles can be distributed and this cell will contribute a factor

$$
\frac{\left(\Re_{\mathrm{k}^{\prime}}-1\right) ! h_{\mathrm{k}^{\prime}} m_{\mathrm{k}^{\prime}}\left(1-h_{\mathrm{k}^{\prime}}\right)^{\mathscr{N}_{\mathrm{k}^{\prime}-m_{\mathrm{k}^{\prime}}}}}{\left(m_{\mathrm{k}^{\prime}}-1\right) !\left(\Re_{\mathrm{k}^{\prime}}-m_{\mathrm{k}^{\prime}}\right) !}
$$

to the weight for a given distribution of $m_{\mathrm{k}}$ 's. It follows that

$$
W_{N, \mathrm{k}^{\prime}}=\sum_{\left(\Sigma m_{\mathrm{k}}=N\right)} \frac{m_{\mathrm{k}^{\prime}}}{\mathscr{T}_{\mathrm{k}^{\prime}}} W\left(m_{\mathrm{k}}\right)=h_{\mathrm{k}^{\prime}} W_{N} .
$$

The last equality holds except for terms which vanish in the limit of a large system because the state vector $\Psi$ gives a probability $\left\langle m_{\mathrm{k}^{\prime}} / \mathscr{N}_{\mathrm{k}^{\prime}}\right\rangle_{\mathrm{Av}}=h_{\mathrm{k}^{\prime}}$ that a given state in $\Delta \mathbf{k}^{\prime}$ is occupied. Now the weights for different numbers of pairs in $\Psi$ are strongly peaked about the most probable number $N$ and therefore the average over distributions with exactly $N$ pairs is essentially equal to the average over all distributions. Since all terms in the wave function come in with a positive sign, it follows that the normalized $\Psi_{N}$ may be decomposed in the form

$$
\Psi_{N}=h_{\mathrm{k}^{\prime}}{ }^{\frac{1}{2}} \varphi_{N 1}+\left(1-h_{\mathrm{k}^{\prime}}\right)^{\frac{1}{2}} \varphi_{N 0},
$$

where $\varphi_{N 1}$ and $\varphi_{N 0}$ are normalized functions.

For purposes of calculating matrix elements and

${ }^{32}$ It is easily seen that $\Psi_{N}$ has zero total spin, corresponding to a singlet state. interaction energies, a further decomposition into states in which occupancy of two pair states, $\mathbf{k}$ and $\mathbf{k}^{\prime}$, is specified is often convenient. Thus we may write:

$$
\begin{array}{r}
\Psi_{N}=\left(h h^{\prime}\right)^{\frac{1}{2}} \varphi_{N 11}+\left[h\left(1-h^{\prime}\right)\right]^{\frac{1}{2}} \varphi_{N 10}+\left[(1-h) h^{\prime}\right]^{\frac{1}{2}} \varphi_{N 01} \\
+\left[(1-h)\left(1-h^{\prime}\right)\right]^{\frac{1}{2}} \varphi_{N 00},
\end{array}
$$

where the first index gives the occupancy of $\mathbf{k}$ and the second of $\mathbf{k}^{\prime}$. It follows from the definition of the functions that

$$
b_{\mathrm{k}^{\prime}} * b_{\mathrm{k}} \varphi_{N 10}=\varphi_{N 01} \text {. }
$$

Thus the diagonal matrix element of $b_{\mathbf{k}}, * b_{\mathrm{k}}$ is

$$
\left(\Psi_{N}\left|b_{\mathrm{k}^{\prime}} * b_{\mathrm{k}}\right| \Psi_{N}\right)=\left[h_{\mathrm{k}}\left(1-h_{\mathrm{k}}\right) h_{\mathrm{k}^{\prime}}\left(1-h_{\mathrm{k}^{\prime}}\right)\right]^{\frac{1}{2}} \text {. }
$$

\section{Ground-State Energy}

If the wave function (2.24) is used as a variational approximation to the true ground-state function, the ground-state energy relative to the energy of the Fermi sea is given by

$$
W_{0}=\left(\Psi_{0}, H_{\text {red }} \Psi_{0}\right),
$$

where $\Psi_{0}$ is the $N$-pair function $\Psi_{N}$, for the ground state. The Bloch energies, $\epsilon_{\mathrm{k}}$, are measured with respect to the Fermi energy and

$$
\begin{aligned}
-V_{\mathbf{k k}^{\prime}}=\left(-\mathbf{k}^{\prime} \downarrow, \mathbf{k}^{\prime} \uparrow \mid\right. & \left.H_{I} \mid-\mathbf{k} \downarrow, \mathbf{k} \uparrow\right) \\
& +\left(\mathbf{k}^{\prime} \uparrow,-\mathbf{k}^{\prime} \downarrow\left|H_{I}\right| \mathbf{k} \uparrow,-\mathbf{k} \downarrow\right) .
\end{aligned}
$$

The decomposition (2.23) leads to the Bloch energy contribution to $W_{0}$ of the form

$$
W_{\mathrm{KE}}=2 \sum_{k>k_{F}} \epsilon_{\mathrm{k}} h_{\mathrm{k}}+2 \sum_{k<k_{F}}\left|\epsilon_{\mathrm{k}}\right|\left(1-h_{\mathrm{k}}\right),
$$

where "KE" stands for kinetic energy. The matrix elements of the interaction term in $H_{\text {red }}$ are given by (2.27) and the interaction energy is

$$
W_{I}=-\sum_{\mathbf{k}, \mathbf{k}^{\prime}} V_{\mathrm{kk}}\left[h_{\mathrm{k}}\left(1-h_{\mathrm{k}}\right) h_{\mathrm{k}^{\prime}}\left(1-h_{\mathrm{k}^{\prime}}\right)\right]^{\frac{1}{2}}
$$

and therefore

$$
\begin{array}{r}
W_{0}=W_{\mathrm{kE}}+W_{I}=2 \sum_{k>k_{F}} \epsilon_{\mathrm{k}} h_{\mathrm{k}}+2 \sum_{k<k_{F}}\left|\epsilon_{\mathrm{k}}\right|\left(1-h_{\mathrm{k}}\right) \\
-\sum_{\mathbf{k}, \mathbf{k}^{\prime}} V_{\mathrm{kk}^{\prime}}\left[h_{\mathrm{k}}\left(1-h_{\mathrm{k}}\right) h_{\mathrm{k}^{\prime}}\left(1-h_{\mathrm{k}^{\prime}}\right)\right]^{\frac{1}{2}} .
\end{array}
$$

By minimizing $W_{0}$ with respect to $h_{\mathrm{k}}$, we are led to an integral equation determining the distribution function:

$$
\frac{\left[h_{\mathrm{k}}\left(1-h_{\mathrm{k}}\right)\right]^{\frac{1}{2}}}{1-2 h_{\mathrm{k}}}=\frac{\sum_{\mathrm{k}^{\prime}} V_{\mathrm{kk}^{\prime}}\left[h_{\mathrm{k}^{\prime}}\left(1-h_{\mathrm{k}^{\prime}}\right)\right]^{\frac{1}{2}}}{2 \epsilon_{\mathrm{k}}} .
$$

We shall neglect anisotropic effects and assume for simplicity that the matrix element $V_{\mathrm{kk}^{\prime}}$ can be replaced by a constant average matrix element,

$$
V=\left\langle V_{\mathrm{kk}^{\prime}}\right\rangle_{\mathrm{Av}}
$$

for pairs making transitions in the region $-\hbar \omega<\epsilon<\hbar \omega$ 
and by zero outside this region, where $\omega$ is the average phonon frequency. This cutoff corresponds to forming our wave function from states in the region where the interaction is expected to be attractive and not mixing in states outside this region. The average is primarily one over directions of $\mathbf{k}$ and $\mathbf{k}^{\prime}$ since the interaction is insensitive to the excitation energy for those transitions of importance in describing the superconducing phase. The average may also be viewed as choosing $h$ to be a function of energy alone, thus neglecting the details of band structure. The laws of similarity indicate this to be a reasonable assumption and the good agreement of our theory with a wide class of superconductors supports this view.

Introduction of the average matrix element into (2.33) leads to

and

$$
h_{\mathrm{k}}=\frac{1}{2}\left[1-\frac{\epsilon_{\mathrm{k}}}{\left(\epsilon_{\mathrm{k}}{ }^{2}+\epsilon_{0}^{2}\right)^{\frac{1}{2}}}\right]
$$

where

$$
\left[h_{\mathrm{k}}\left(1-h_{\mathrm{k}}\right)\right]^{\frac{1}{2}}=\frac{\epsilon_{0}}{\left.2\left(\epsilon_{\mathrm{k}}{ }^{2}+\epsilon_{0}\right)^{2}\right)^{\frac{1}{2}}},
$$

$$
\epsilon_{0}=V \sum_{\mathrm{k}^{\prime}}\left[h_{\mathrm{k}^{\prime}}\left(1-h_{\mathrm{k}^{\prime}}\right)\right]^{\frac{1}{2}},
$$

the sum extending over states within the range $\left|\epsilon_{\mathrm{k}}\right|$ $<\hbar \omega$. If (2.36) and (2.37) are combined, one obtains a condition on $\epsilon_{0}$ :

$$
\frac{1}{V}=\sum_{\mathbf{k}} \frac{1}{2\left(\epsilon_{\mathrm{k}}^{2}+\epsilon_{0}^{2}\right)^{\frac{2}{2}}} .
$$

Replacing the sum by an integral and recalling that $V=0$ for $\left|\epsilon_{\mathrm{k}}\right|>\hbar \omega$, we may replace this condition by

$$
\frac{1}{N(0) V}=\int_{0}^{\hbar \omega} \frac{d \epsilon}{\left(\epsilon^{2}+\epsilon_{0}^{2}\right)^{\frac{1}{2}}}
$$

Solving for $\epsilon_{0}$, we obtain

$$
\epsilon_{0}=\hbar \omega / \sinh \left[\frac{1}{N(0) V}\right] .
$$

where $N(0)$ is the density of Bloch states of one spin per unit energy at the Fermi surface.

The ground state energy is obtained by combining the expressions for $h_{\mathrm{k}}$ and $\epsilon_{0},(2.35),(2.36)$, and (2.37), with (2.32). We find

$$
\begin{aligned}
W_{0}=4 N(0) & \int_{0}^{\hbar \omega} \epsilon h(\epsilon) d \epsilon-\frac{\epsilon_{0}^{2}}{V} \\
& =2 N(0) \int_{0}^{\hbar \omega}\left[\epsilon-\frac{\epsilon^{2}}{\left(\epsilon^{2}+\epsilon_{0}^{2}\right)^{\frac{1}{2}}}\right] d \epsilon-\frac{\epsilon_{0}^{2}}{V},
\end{aligned}
$$

where we have used the fact that $[1-h(-\epsilon)]=h(\epsilon)$, that is, the distribution function is symmetric in electrons and holes with respect to the Fermi surface.
Using the relations (2.40), we find that the difference in energy between the superconducting and normal states at the absolute zero becomes

$$
W_{0}=N(0)(\hbar \omega)^{2}\left\{1-\left[1+\left(\frac{\epsilon_{0}}{\hbar \omega}\right)^{2}\right]^{\frac{1}{2}}\right\}=\frac{-2 N(0)(\hbar \omega)^{2}}{e^{[2 / N(0) V]}-1} .
$$

If there is a net negative interaction on the average, no matter how weak, there exists a coherent state which is lower in energy than the normal state. Thus our criterion for superconductivity is that $V>0$, as given in (2.6).

For excitations which are small compared to $\hbar \omega$, the phonon interaction is essentially independent of isotopic mass and therefore the total mass dependence of $W_{0}$ comes from $(\hbar \omega)^{2}$, in agreement with the isotope effect. Empirically, $W_{0}$ is of the order of $N(0)\left(k T_{c}\right)^{2}$ and in general $k T_{c}$ is much less than $\hbar \omega$. According to (2.42), this will occur if $N(0) V<1$, that is, the weak coupling limit.

It should be noted that the ground state energy cannot be obtained in any finite-order perturbation theory. In the strong-coupling limit, (2.42) gives the correct result, $-N(0)(\hbar \omega)^{2} V$, for the average interaction approximation and it is possible that our solution is accurate in the statistical limit over the entire range of coupling. (See Appendix A.)

In the weak-coupling limit, the energy becomes

$$
W_{0}=-2 N(0)(\hbar \omega)^{2} \exp \left[-\frac{2}{N(0) V}\right] .
$$

which may be expressed in terms of the number of electrons, $n_{c}$, in pairs virtually excited above the Fermi surface as

where

$$
W_{0}=-\frac{1}{2} n_{c}^{2} / N(0),
$$

$$
n_{c}=2 N(0) \hbar \omega \exp \left[-\frac{1}{N(0) V}\right] \text {. }
$$

In this form, the cooperative nature of the ground state is evident. Using the empirical order of magnitude relation between $W_{0}$ and $k T_{c}$, we might estimate

$$
k T_{c} \sim \hbar \omega \exp \left[-\frac{1}{N(0) V}\right] .
$$

In the next chapter we shall see that the explicit calculation of $k T_{c}$ from the free energy as a function of temperature leads to nearly this result.

\section{Energy gap at $T=0^{\circ} \mathrm{K}$}

An important feature of the reduced Hamiltonian is that there are no excitations from the ground state, analogous to single-particle excitations of the Bloch theory, with vanishing excitation energy. This is easily 
seen by considering a function

$\Psi_{\mathrm{exe}}=\left\{\prod_{\mathrm{k} \neq \mathbf{k}^{\prime}, \mathrm{k}^{\prime \prime}}\left[\left(1-h_{\mathrm{k}}\right)^{\frac{1}{2}}+h_{\mathrm{k}^{\mathrm{z}}} \frac{{ }^{\frac{1}{2}} b_{\mathrm{k}}}{}{ }^{*}\right]\right\} c_{-\mathrm{k}^{\prime} \downarrow}{ }^{*} c_{\mathrm{k}^{\prime \prime} \uparrow}{ }^{*} \Phi_{0}$,

which is orthogonal to the ground state function and corresponds to breaking up a pair in $\mathbf{k}^{\prime}$, the spin-up member going to $\mathbf{k}^{\prime \prime} \uparrow$. The projection of $\Psi_{\text {exc }}$ onto the space with $N$ pairs is also orthogonal to $\Psi_{0}$. The decomposition of $\Psi_{N \text { ex }}$ is the same as that of $\Psi_{0}$, except that $-\mathbf{k}^{\prime} \downarrow$ and $\mathbf{k}^{\prime \prime} \uparrow$ are definitely known to be occupied and $\mathbf{k}^{\prime} \uparrow$ and $-\mathbf{k}^{\prime \prime} \downarrow$ are unoccupied. This leads to the excitation energy

$$
\begin{array}{r}
W_{\mathbf{k}^{\prime}, \mathrm{k}^{\prime \prime}}-W_{0}=\epsilon_{\mathrm{k}^{\prime}}\left(1-2 h_{\mathrm{k}^{\prime}}\right)+\epsilon_{\mathrm{k}^{\prime \prime}}\left(1-2 h_{\mathrm{k}^{\prime \prime}}\right) \\
+2 V \sum_{\mathbf{k}}\left[h_{\mathbf{k}}\left(1-h_{\mathrm{k}}\right)\right]^{\frac{1}{2}}\left\{\left[h_{\mathrm{k}^{\prime}}\left(1-h_{\mathrm{k}^{\prime}}\right)\right]^{\frac{1}{3}}\right. \\
\left.+\left[h_{\mathrm{k}^{\prime \prime}}\left(1-h_{\mathbf{k}^{\prime \prime}}\right)\right]^{\frac{1}{2}}\right\},
\end{array}
$$

the decrease in interaction energy arising from the fact that pairs cannot make transitions into or out of pair states $\mathbf{k}^{\prime}$ and $\mathbf{k}^{\prime \prime}$ in the excited function because these states are occupied by single particles. Combining (2.35), (2.36), and (2.37) with (2.48), we find

$$
\begin{array}{r}
W_{\mathrm{k}^{\prime}, \mathrm{k}^{\prime \prime}}-W_{0}=\frac{\epsilon_{\mathrm{k}^{\prime}}{ }^{2}}{E_{\mathrm{k}^{\prime}}}+\frac{\epsilon_{\mathrm{k}^{\prime \prime}}{ }^{2}}{E_{\mathrm{k}^{\prime \prime}}}+\epsilon_{0}^{2}\left(\frac{1}{E_{\mathrm{k}^{\prime}}}+\frac{1}{E_{\mathrm{k}^{\prime \prime}}}\right) \\
=E_{\mathrm{k}^{\prime}}+E_{\mathrm{k}^{\prime \prime}},
\end{array}
$$

where

$$
E_{\mathrm{k}}=\left(\epsilon_{\mathrm{k}}{ }^{2}+\epsilon_{0}^{2}\right)^{\frac{1}{2}} .
$$

When $\epsilon_{\mathrm{k}} \rightarrow 0$, then $E_{\mathrm{k}} \rightarrow \epsilon_{0}$ and (2.49) shows that the minimum excitation energy is $2 \epsilon_{0}$. These single-particlelike excitations have the new dispersion law (2.50) which goes over to the normal law when $\epsilon_{\mathrm{k}} \gg \epsilon_{0}$.

To obtain a complete set of excitations, we must include excited state pair functions generated by

$$
\left[\left(1-h_{\mathrm{k}}\right)^{\frac{1}{2}} b_{\mathrm{k}}{ }^{*}-h_{\mathrm{k}}^{\frac{1}{2}}\right] \text {, }
$$

which by construction are orthogonal to the ground state pair functions generated by

$$
\left[\left(1-h_{\mathrm{k}}\right)^{\frac{1}{2}}+h_{\mathrm{k}^{\frac{1}{2}}} b_{\mathrm{k}}{ }^{*}\right] \text {. }
$$

The decomposition of an excited state with an excited pair in $\mathbf{k}^{\prime}$ and a ground pair in $\mathbf{k}$ would be

$$
\begin{aligned}
\Psi_{\mathrm{k}^{\prime}} & =\left[h_{\mathrm{k}}\left(1-h_{\mathrm{k}^{\prime}}\right)\right]^{\frac{1}{2}} \varphi_{11}-\left[h_{\mathrm{k}} h_{\mathrm{k}^{\prime}}\right]^{\frac{1}{2}} \varphi_{10} \\
& +\left[\left(1-h_{\mathrm{k}}\right)\left(1-h_{\mathrm{k}^{\prime}}\right)\right]^{\frac{1}{2}} \varphi_{01}-\left[\left(1-h_{\mathrm{k}}\right) h_{\mathrm{k}^{\prime}}\right]^{\frac{1}{2}} \varphi_{00},
\end{aligned}
$$

where the functions $\varphi$ are normalized and the second script denotes the occupancy of $\mathbf{k}^{\prime}$. Taking the expectation value of $H_{\text {red }}$ with respect to (2.53), we find the energy to form an excited pair in state $\mathbf{k}^{\prime}$ is :

$$
\begin{aligned}
W_{\mathbf{k}^{\prime}}- & W_{0}=2 \epsilon_{\mathrm{k}^{\prime}}\left(1-2 h_{\mathrm{k}^{\prime}}\right) \\
& +4 V \sum_{\mathrm{k}}\left[h_{\mathrm{k}}\left(1-h_{\mathrm{k}}\right) h_{\mathrm{k}^{\prime}}\left(1-h_{\mathrm{k}^{\prime}}\right)\right]^{\frac{1}{2}}=2 E_{\mathrm{k}^{\prime}} .
\end{aligned}
$$

Again the minimum energy required to form an excitation is $2 \epsilon_{0}$ and an energy gap of width $2 \epsilon_{0}$ appears in the excitation spectrum in a natural way. It follows that in general the energy difference between two states, 1 and 2, is given by the difference in the sums of the excited-particle energies,

$$
W_{1}-W_{2}=\sum_{1} E_{k}-\sum_{2} E_{k}
$$

and it is unnecessary to distinguish between single particles and two members of an excited pair in calculating the sums.

Collective excitations corresponding to long-range density fluctuations are suppressed by the subsidiary condition on the wave function resulting from the collective description of the electron-ion interaction. ${ }^{16}$ The effect of the terms neglected in the Hamiltonian, $H-H_{\text {red }}=H^{\prime}$, can be estimated by a perturbation expansion of $H^{\prime}$ in eigenfunctions of $H_{\text {red }}$. This expansion is carried out to second order in Appendix A and it is concluded that $H^{\prime}$ will contribute little to the condensation energy. The shift in the zero-point energy of the lattice associated with the transition at the absolute zero is estimated in Appendix B and it is shown that this effect contributes a small correction to $W_{0}$. The electron self-energy shift has not been calculated at the present time; however, it is also believed that the correction is small.

\section{EXCITED STATES}

An excited state of the system will be formed by specifying the set of states, $s$, which are occupied by single particles and the set of states, $\odot$, occupied by excited pairs. The rest of the states, $\mathcal{G}$, will be available for occupation by ground pairs. The term "singleparticle" occupation means that either $\mathbf{k} \uparrow$ or $-\mathbf{k} \downarrow$ is occupied by an electron, but not both. "Excited pair" and "ground pair" occupation refers to pairs which are in functions generated by operators of the form (2.51) and (2.52) respectively. Wave functions with different distributions of single particles and excited pairs are orthogonal to each other and the totality of such functions constitutes a complete set of excited states which are in one-to-one correspondence with the Bloch-type excitations in the normal metal.

The energy of the excited states will be evaluated by using the reduced Hamiltonian plus the Bloch energy for single particles:

$$
\begin{aligned}
H_{e}=\sum_{k>k_{F}, \sigma} \epsilon_{\mathrm{k}} n_{\mathrm{k} \sigma}+\sum_{k<k_{F}, \sigma}\left|\epsilon_{\mathrm{k}}\right|\left(1-n_{\mathrm{k} \sigma}\right) & \\
& -\sum_{\mathbf{k}, \mathbf{k}^{\prime}} V_{\mathbf{k} \mathbf{k}^{\prime}} b_{\mathbf{k}^{\prime}}, b_{\mathbf{k}},
\end{aligned}
$$

where the second term gives the Bloch energy of the holes and the energies $\epsilon_{\mathrm{k}}$ are measured relative to the Fermi energy. Since $H_{I}$ contains only terms for transitions by ground pairs and excited pairs, the single particles contribute only to the Bloch energy and hence they are treated as in the Bloch scheme.

The equilibrium condition of the system at a specified temperature will be determined by minimizing the free 
energy with respect to the distribution function for excited particles, $f$, and ground pairs, $h$.

It turns out that $h_{\mathrm{k}}$ is a function of temperature and therefore excited and ground pairs are not necessarily orthogonal to each other at different temperatures, although the excited states form a complete orthonormal set at each temperature. The most probable distribution of single particles also varies with temperature and thus the great majority of states contributing to the free energy at different temperatures will be orthogonal in any event. The situation is similar to taking the lattice constant temperature dependent as a result of thermal expansion. This freedom in choosing $h_{\mathrm{k}}$ allows us to work in that representation which minimizes the free energy at the specified temperature.

A typical excited state wave function can be written as the projection of

$$
\begin{array}{r}
\Psi_{\mathrm{exc}}=\prod_{\mathbf{k}(\mathcal{G})}\left[\left(1-h_{\mathrm{k}}\right)^{\frac{1}{2}}+h_{\mathrm{k}^{\frac{2}{2}} b_{\mathrm{k}}} *\right] \prod_{\mathbf{k}^{\prime}(\rho)}\left[\left(1-h_{\mathrm{k}^{\prime}}\right)^{\frac{1}{2}} b_{\mathbf{k}^{\prime}} *\right. \\
\left.-h_{\mathbf{k}^{\prime}}{ }^{\frac{1}{2}}\right] \prod_{\mathbf{k}^{\prime \prime}(\mathcal{S})} c_{\left(\mathrm{k}^{\prime \prime}\right)}{ }^{*} \Phi_{0},
\end{array}
$$

onto the space with $N$ pairs, where $\mathcal{G}, \mathcal{P}$, and $S$ specify the states occupied by ground pairs, excited pairs, and single particles respectively and $c_{\left(\mathrm{k}^{\prime \prime}\right)} *$ denotes either $\mathbf{k}^{\prime \prime} \uparrow$ or $-\mathbf{k}^{\prime \prime} \downarrow$ is included in the product. For any specified $\mathbf{k}$, this function can be decomposed into a portion with $\mathbf{k}$ occupied and a portion with $\mathbf{k}$ unoccupied. The decompositions for the three cases in which $\mathbf{k}$ is in the sets $\mathcal{G}, \mathcal{P}$, and $S$ are

Ground :

$$
\Psi=h_{\mathrm{k}}^{\frac{1}{2}} \varphi_{1}\left(\cdots 1_{\mathrm{k}} \cdots\right)+\left(1-h_{\mathrm{k}}\right)^{\frac{1}{2}} \varphi_{0}\left(\cdots 0_{\mathrm{k}} \cdots\right),
$$

Excited :

$$
\Psi=\left(1-h_{\mathrm{k}}\right)^{\frac{1}{2}} \varphi_{1}\left(\cdots 1_{\mathrm{k}} \cdots\right)-h_{\mathrm{k}}^{\frac{1}{2}} \varphi_{0}\left(\cdots 0_{\mathrm{k}} \cdots\right),
$$

Single in $\mathbf{k} \uparrow$ :

$$
\Psi=c_{\mathrm{k}} \uparrow^{*} \varphi_{0}\left(\cdots 0_{\mathrm{k}} \cdots\right),
$$

where the $\varphi$ 's are normalized functions with $1_{\mathrm{k}}$ representing pair state $k$ being occupied and $0_{\mathrm{k}}$ unoccupied.

To determine the distribution functions, we need the free energy

$$
F=W-T S,
$$

where $W$ is the energy calculated by an ensemble average over the wave functions of the form (3.2) and $S$ is the entropy.

To enumerate the systems in the ensemble we divide $k$-space into cells $\Delta \mathbf{k}$ containing $\mathfrak{T}_{\mathrm{k}}$ pair states as before. Let there be $S_{\mathrm{k}}$ single particles and $P_{\mathrm{k}}$ excited pairs in $\Delta \mathbf{k}$, with the rest of the $\mathscr{N}_{\mathbf{k}}$ states being occupied by ground pairs. The probability that either $\mathbf{k} \uparrow$ or $-\mathbf{k} \downarrow$ is occupied by a single particle is $s_{\mathrm{k}}=S_{\mathrm{k}} / \mathscr{T}_{\mathrm{k}}$, while the probability for an excited pair in state $\mathbf{k}$ is $p_{\mathrm{k}}=P_{\mathrm{k}} / \mathscr{T}_{\mathrm{k}}$ and therefore the probability for a ground pair is $\left(1-s_{\mathrm{k}}-p_{\mathrm{k}}\right)$. Above the Fermi surface, $h<\frac{1}{2}$ and $s$ and $p$ refer to excited electrons; below the Fermi surface, $h>\frac{1}{2}$ and $s$ and $p$ refer to holes.

The diagonal element of $n_{\mathrm{k} \sigma}$ follows immediately from (3.3), (3.4), and (3.5). Including the factor giving fractional number of configurations for which each decomposition applies, we have

$$
\begin{aligned}
\left(\psi\left|n_{\mathrm{k} \sigma}\right| \psi\right) & =(1 / 2) s_{\mathrm{k}}+p_{\mathrm{k}}\left(1-h_{\mathrm{k}}\right) \\
& +\left(1-s_{\mathrm{k}}-p_{\mathrm{k}}\right) h_{\mathrm{k}}, \quad \epsilon>0 ; \\
\left(\psi\left|1-n_{\mathrm{k} \sigma}\right| \psi\right)=(1 / 2) s_{\mathrm{k}}+p_{\mathrm{k}} h_{\mathrm{k}} & \\
+\left(1-s_{\mathrm{k}}-p_{\mathrm{k}}\right)\left(1-h_{\mathrm{k}}\right), & \epsilon<0 .
\end{aligned}
$$

Upon using (3.7) and the fact that $1-h_{\mathrm{k}}(-\epsilon)=h_{\mathrm{k}}(\epsilon)$, the Bloch energy contribution to $W$,

$$
\left(\psi\left|\sum_{\mathrm{k}>k_{F} \sigma} \epsilon_{\mathrm{k}} n_{\mathrm{k} \sigma}+\sum_{\mathrm{k}<k_{F} \sigma}\right| \epsilon_{\mathrm{k}}\left|\left(1-n_{\mathrm{k} \sigma}\right)\right| \psi\right),
$$

becomes

$W_{\mathrm{KE}}=\sum_{\mathrm{k}}\left|\epsilon_{\mathrm{k}}\right|\left[s_{\mathrm{k}}+2 p_{\mathrm{k}}+2\left(1-s_{\mathrm{k}}-2 p_{\mathrm{k}}\right) h_{\mathrm{k}}\left(\left|\epsilon_{\mathrm{k}}\right|\right)\right]$,

where we have carried out the spin sum.

To calculate the matrix elements of the pair interaction operator $\sum V_{\mathrm{kk}^{\prime}} b_{\mathrm{k}^{\prime}} * b_{\mathrm{k}}$, we assume that $V_{\mathrm{kk}^{\prime}}$ varies continuously with $\mathbf{k}$ and $\mathbf{k}^{\prime}$ so that $V_{\mathbf{k k}^{\prime}}$ may be considered to be the same for all transitions from states in $\Delta \mathbf{k}$ to states in $\Delta \mathbf{k}^{\prime}$. Let $\mathbf{k}$ and $\mathbf{k}^{\prime}$ represent two specified wave vectors in $\Delta \mathbf{k}$ and $\Delta \mathbf{k}^{\prime}$. To obtain nonvanishing matrix elements for $b_{\mathrm{k}}{ }^{*} b_{\mathrm{k}}$, these states must be occupied by either excited $(-)$ or ground $(+)$ pairs, giving the four possibilities,,,++--+--+ for $\mathbf{k}$ and $\mathbf{k}^{\prime}$, respectively. For any one of these cases, a typical wave function may be decomposed into components in which the pair occupancy of $\mathbf{k}$ and $\mathbf{k}^{\prime}$ is specified:

$$
\begin{aligned}
\Psi_{\mathrm{k}, \mathrm{k}^{\prime}}=\alpha_{11} \varphi_{11}( & \left.\cdots 1_{\mathrm{k}} \cdots 1_{\mathrm{k}^{\prime}} \cdots\right) \\
& +\alpha_{10} \varphi_{10}\left(\cdots 1_{\mathrm{k}} \cdots 0_{\mathrm{k}^{\prime}} \cdots\right) \\
& +\alpha_{01} \varphi_{01}\left(\cdots 0_{\mathrm{k}} \cdots 1_{\mathrm{k}^{\prime}} \cdots\right) \\
& +\alpha_{00} \varphi_{00}\left(\cdots 0_{\mathrm{k}} \cdots 0_{\mathrm{k}^{\prime}} \cdots\right)
\end{aligned}
$$

where the $\varphi$ 's are normalized functions. Table I gives the values of the $\alpha$ 's for the different cases along with the fractional number of configurations for which they apply.

The diagonal elements of $b_{\mathbf{k}^{\prime}}{ }^{*} b_{\mathrm{k}}$ are given by $\alpha_{10} \alpha_{01}$ in each case. If we sum these, weighted according to the probability they occur in the ensemble, we obtain

$$
\begin{aligned}
& {\left[h(1-h) h^{\prime}\left(1-h^{\prime}\right)\right]^{\frac{1}{2}}\left\{(1-s-p)\left(1-s^{\prime}-p^{\prime}\right)\right.} \\
& \left.+p p^{\prime}-(1-s-p) p^{\prime}-p\left(1-s^{\prime}-p^{\prime}\right)\right\} \\
& =\left[h(1-h) h^{\prime}\left(1-h^{\prime}\right)\right]^{\frac{1}{2}} \\
& \quad \times\left\{(1-s-2 p)\left(1-s^{\prime}-2 p^{\prime}\right)\right\} .
\end{aligned}
$$

Introducing these matrix elements into the ensemble average of the interaction Hamiltonian, we find

$$
\begin{aligned}
W_{I}=-\sum_{\mathrm{k}, \mathrm{k}^{\prime}} V_{\mathrm{kk}} & {\left[h_{\mathrm{k}}\left(1-h_{\mathrm{k}}\right) h_{\mathrm{k}^{\prime}}\left(1-h_{\mathrm{k}^{\prime}}\right)\right]^{\frac{1}{2}} } \\
& \times\left\{\left(1-s_{\mathrm{k}}-2 p_{\mathrm{k}}\right)\left(1-s_{\mathrm{k}^{\prime}}-2 p_{\mathrm{k}^{\prime}}\right)\right\} .
\end{aligned}
$$


TABLE I. Coefficients for the decomposition of $\Psi$ according to Eq. (3.10).

\begin{tabular}{|c|c|c|c|c|c|c|}
\hline \multicolumn{7}{|c|}{ Wave function } \\
\hline $\mathbf{k}$ & $\mathbf{k}^{\prime}$ & Fractional No. of cases & $\alpha_{11}$ & $\alpha_{10}$ & $\alpha_{01}$ & $\alpha_{00}$ \\
\hline+ & + & $(1-s-p)\left(1-s^{\prime}-p^{\prime}\right)$ & {$\left[h h^{\prime}\right]^{z}$} & {$\left[h\left(1-h^{\prime}\right)\right]^{k}$} & {$\left[(1-h) h^{\prime}\right]^{\frac{1}{2}}$} & {$\left[(1-h)\left(1-h^{\prime}\right)\right]^{\prime}$} \\
\hline- & - & $p p^{\prime}$ & {$\left[(1-h)\left(1-h^{\prime}\right)\right]^{\frac{1}{2}}$} & $-\left[(1-h) h^{\prime}\right]^{\frac{1}{2}}$ & $-\left[h\left(1-h^{\prime}\right)\right]^{\frac{1}{2}}$ & {$\left[h h^{\prime}\right]^{\frac{1}{2}}$} \\
\hline+ & - & $(1-s-p) p^{\prime}$ & {$\left[h\left(1-h^{\prime}\right)\right]^{\frac{1}{2}}$} & $-\left[h h^{\prime}\right]^{\frac{1}{2}}$ & {$\left[(1-h)\left(1-h^{\prime}\right)\right]^{\frac{1}{2}}$} & $-\left[(1-h) h^{\prime}\right]^{\frac{1}{2}}$ \\
\hline - & + & $p\left(1-s^{\prime}-p^{\prime}\right)$ & {$\left[(1-h) h^{\prime}\right]^{k}$} & {$\left[(1-h)\left(1-h^{\prime}\right)\right]^{t}$} & $-\left[h h^{\prime}\right]^{\frac{1}{2}}$ & $-\left[h\left(1-h^{\prime}\right)\right]^{\frac{1}{2}}$ \\
\hline
\end{tabular}

It should be noted that the Bloch energy (3.9) and the interaction energy (3.12) depend on $(s+2 p)$ or the total occupancy probability. The energy does not depend upon the relative probability for single-particle and excited-pair occupation. Thus one may use a distribution function $f$ which gives the over-all probability of occupancy, where

and

$$
s_{\mathrm{k}}=2 f_{\mathrm{k}}\left(1-f_{\mathrm{k}}\right)
$$

$$
p_{\mathrm{k}}=f_{\mathrm{k}}^{2} \text {, }
$$

which follows from the fact that $s_{\mathrm{k}}$ is the probability that either $\mathbf{k} \uparrow$ is occupied and $-\mathbf{k} \downarrow$ is empty or the reverse and $p_{\mathbf{k}}$ is the probability that both $\mathbf{k} \uparrow$ and $-\mathbf{k} \downarrow$ are occupied. The free energy can be minimized directly with respect to $h_{\mathrm{k}}, p_{\mathrm{k}}$, and $s_{\mathrm{k}}$ without introducing $f_{\mathrm{k}}$ and one indeed finds that (3.13) and (3.14) hold.

Since the excited particles are specified independently for each system in the ensemble, the usual expression for the entropy in terms of $f$ may be used:

$-T S=2 k T \sum_{\mathrm{k}^{\prime}}\left\{f_{\mathrm{k}^{\prime}} \ln f_{\mathrm{k}^{\prime}}+\left(1-f_{\mathrm{k}^{\prime}}\right) \ln \left(1-f_{\mathrm{k}^{\prime}}\right)\right\}$.

\section{Minimization of the Free Energy}

If the expressions (3.13) and (3.14) are introduced into (3.9) and (3.12), the free energy becomes

$$
\begin{aligned}
& F=2 \sum_{\mathbf{k}}\left|\epsilon_{\mathrm{k}}\right|\left[f_{\mathrm{k}}+\right.\left.\left(1-2 f_{\mathrm{k}}\right) h_{\mathrm{k}}\left(\left|\epsilon_{\mathrm{k}}\right|\right)\right] \\
&-\sum_{\mathbf{k}, \mathbf{k}^{\prime}} V_{\mathbf{k k}^{\prime}}\left[h_{\mathrm{k}}\left(1-h_{\mathrm{k}}\right) h_{\mathrm{k}^{\prime}}\left(1-h_{\mathrm{k}^{\prime}}\right)\right]^{\frac{1}{3}} \\
& \quad \times\left\{\left(1-2 f_{\mathrm{k}}\right)\left(1-2 f_{\mathrm{k}^{\prime}}\right)\right\}-T S .
\end{aligned}
$$

When we minimize $F$ with respect to $h_{\mathrm{k}}$, we find that

$$
\begin{aligned}
2 \epsilon_{\mathrm{k}}-\sum_{\mathbf{k}^{\prime}} V_{\mathrm{kk}^{\prime}}\left[h_{\mathrm{k}^{\prime}}\left(1-h_{\mathrm{k}^{\prime}}\right)\right]^{\frac{1}{2}}\left(1-2 f_{\mathrm{k}^{\prime}}\right) & \\
& \times \frac{\left(1-2 h_{\mathrm{k}}\right)}{\left[h_{\mathrm{k}}\left(1-h_{\mathrm{k}}\right)\right]^{\frac{1}{2}}}=0,
\end{aligned}
$$

or

$$
\frac{\left[h_{\mathrm{k}}\left(1-h_{\mathrm{k}}\right)\right]^{\frac{1}{2}}}{1-2 h_{\mathrm{k}}}=\sum_{\mathrm{k}^{\prime}} \frac{V_{\mathrm{kk}^{\prime}}\left[h_{\mathrm{k}^{\prime}}\left(1-h_{\mathrm{k}^{\prime}}\right)\right]^{\frac{1}{2}}\left(1-2 f_{\mathrm{k}^{\prime}}\right)}{2 \epsilon_{\mathrm{k}}},
$$

where the energy $\epsilon_{\mathrm{k}}$ is measured relative to the Fermi energy and $\epsilon_{\mathrm{k}}<0$ for $k \leqslant k_{F}$. Assuming as before that the interaction can be replaced by a constant average matrix element $-V$, defined by (2.34) for $\left|\epsilon_{\mathrm{k}}\right|<\hbar \omega$ and by zero outside this region, it follows that $h_{\mathrm{k}}$ is again of the form

and

$$
h_{\mathrm{k}}=\frac{1}{2}\left[1-\left(\epsilon_{\mathrm{k}} / E_{\mathrm{k}}\right)\right] \text {, }
$$

$$
\left[h_{\mathrm{k}}\left(1-h_{\mathrm{k}}\right)\right]^{\frac{1}{2}}=\frac{1}{2} \epsilon_{0} / E_{\mathrm{k}} \text {. }
$$

The energy $E_{\mathrm{k}}$, a positive definite quantity, is defined as

where

$$
E_{\mathrm{k}}=+\left(\epsilon_{\mathrm{k}}^{2}+\epsilon_{0}^{2}\right)^{\frac{1}{2}}
$$

$$
\epsilon_{0}=V \sum_{\mathrm{k}^{\prime}}\left[h_{\mathrm{k}^{\prime}}\left(1-h_{\mathrm{k}^{\prime}}\right)\right]^{\frac{1}{2}}\left(1-2 f_{\mathrm{k}^{\prime}}\right) .
$$

It will turn out that $2 \epsilon_{0}$ is the magnitude of the energy gap in the single-particle density of states and therefore the distribution of ground pairs is determined by the magnitude of the gap at that temperature.

When we minimize $F$ with respect to $f_{\mathrm{k}}$, we find that

$$
\begin{gathered}
2 \epsilon_{\mathrm{k}}\left(1-2 h_{\mathrm{k}}\right)+4 \sum_{\mathrm{k}^{\prime}} V_{\mathrm{k} \mathrm{k}^{\prime}}\left[h_{\mathrm{k}^{\prime}}\left(1-h_{\mathrm{k}^{\prime}}\right) h_{\mathrm{k}}\left(1-h_{\mathrm{k}}\right)\right]^{\frac{1}{2}} \\
\times\left(1-2 f_{\mathrm{k}^{\prime}}\right)+2 k T \ln \left(f_{\mathrm{k}} /\left(1-f_{\mathrm{k}}\right)\right)=0,
\end{gathered}
$$

and using (3.19) through (3.22), we find that

$$
-\ln \left(f_{\mathrm{k}} /\left(1-f_{\mathrm{k}}\right)\right)=\beta\left[\frac{\epsilon_{\mathrm{k}}^{2}}{E_{\mathrm{k}}}+\frac{\epsilon_{0}^{2}}{E_{\mathrm{k}}}\right]=\beta E_{\mathrm{k}},
$$

where $\beta=1 / k T$ and $E_{\mathrm{k}}$ is a positive quantity. The solution for $f_{k}$ is

$$
f_{\mathrm{k}}=\frac{1}{e^{\beta E_{\mathrm{k}}}+1}=f\left(E_{\mathrm{k}}\right)
$$

Thus the single particles and excited pairs describe a set of independent fermions with the modified dispersion law (3.21). For $k>k_{F}, f_{\mathrm{k}}$ specifies electron occupation while for $k<k_{F}, f_{\mathrm{k}}$ specifies hole occupation. These electrons and holes are identified with the normal component of the two-fluid model.

When $\epsilon_{\mathrm{k}} \rightarrow 0^{+}$, then $E_{\mathrm{k}} \rightarrow \epsilon_{0}$ for the electron and when $\epsilon_{\mathrm{k}} \rightarrow 0^{-}$, then $E_{\mathrm{k}} \rightarrow \epsilon_{0}$ for the hole or the corresponding electron energy $\rightarrow-\epsilon_{0}$. Thus the new density of states has an energy gap of magnitude $2 \epsilon_{0}$ centered about the Fermi energy. The modified density of states is given by

$$
\frac{d N(E)}{d E}=\frac{d N(\epsilon)}{d \epsilon} \frac{d \epsilon}{d E}=N(0) \frac{E}{\left(E^{2}-\epsilon_{0}^{2}\right)^{\frac{1}{2}}},
$$

which is singular at the edges of the gap, $E=\epsilon_{0}$. The total number of states is of course unaltered by the interaction.

If the distribution functions (3.19) and (3.25) are introduced, the condition determining the energy gap 


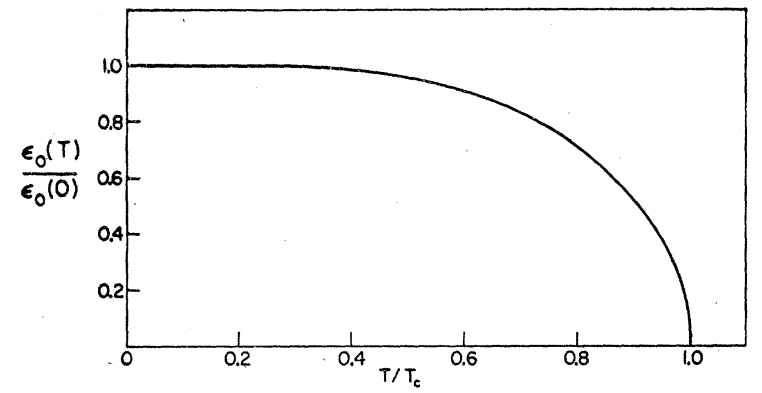

FIG. 1. Ratio of the energy gap for single-particle-like excitations to the gap at $T=0^{\circ} \mathrm{K} v s$ temperature.

(3.22) becomes (dividing by $\epsilon_{0}$ )

$$
\frac{1}{N(0) V}=\int_{0}^{\hbar \omega} \frac{d \epsilon}{\left(\epsilon^{2}+\epsilon_{0}^{2}\right)^{\frac{1}{2}}} \tanh \left[\frac{1}{2} \beta\left(\epsilon^{2}+\epsilon_{0}^{2}\right)^{\frac{1}{2}}\right]
$$

where we have replaced the sum by an integral and used the fact that the distribution functions are symmetric in holes and electrons with respect to the Fermi energy. The transition temperature, $T_{c}$, is defined as the boundary of the region beyond which there is no real, positive $\epsilon_{0}$ which satisfies (3.27). Above $T_{c}$ therefore, $\epsilon_{0}=0$ and $f\left(E_{\mathrm{k}}\right)$ becomes $f\left(\epsilon_{\mathrm{k}}\right)$, so that the metal returns to the normal state. Below $T_{c}$ the solution of (3.27), $\epsilon_{0} \neq 0$, minimizes the free energy and we have the superconducting phase. Thus (3.26) can be used to determine the critical temperature and we find

or

$$
\frac{1}{N(0) V}=\int_{0}^{\hbar \omega} \frac{d \epsilon}{\epsilon} \tanh \left(\frac{1}{2} \beta_{c} \epsilon\right),
$$

$$
k T_{c}=1.14 \hbar \omega \exp \left[-\frac{1}{N(0) V}\right]
$$

as long as $k T_{c} \ll \hbar \omega$, which corresponds to the weakcoupling case discussed in Sec. II. The transition temperature is proportional to $\hbar \omega$, which is consistent with the isotope effect. The small magnitude of $T_{c}$ compared to the Debye temperature is presumably due to the cancellation of the phonon interaction and the screened Coulomb interaction for transitions of importance in describing the superconducting state, and the resulting effect of the exponential.

The transition temperature is a strong function of the electron concentration since the density of states enters exponentially. It should be possible to make estimates of the change in transition temperature with pressure, alloying, etc., from (3.29).

A plot of the energy gap as a function of temperature is given in Fig. 1. The ratio of the energy gap at $T=0^{\circ} \mathrm{K}$ to $k T_{c}$ is given by combining (2.36) and (3.28):

$$
2 \epsilon_{0} / k T_{c}=3.50 \text {. }
$$

From the law of corresponding states, this ratio is predicted to be the same for all superconductors. Near
$T_{c}$, the gap may be expressed as

$$
\epsilon_{0}=3.2 k T_{c}\left[1-\left(T / T_{c}\right)\right]^{\frac{1}{2}},
$$

which has the form suggested by Buckingham..$^{33}$

It can be seen from the distribution functions that our theory goes over into the Bloch scheme above the transition temperature. As $T \rightarrow T_{c}, E_{\mathrm{k}} \rightarrow\left|\epsilon_{\mathrm{k}}\right|$, and $h_{\mathrm{k}}$ vanishes for $k>k_{F}$ and is unity for $k<k_{F}$. According to (3.4) the excited-pair function specifies complete electron occupancy for $k>k_{F}$ and complete hole occupancy for $k<k_{F}$ in this case. Thus, in the normal state, the ground pairs vanish above the Fermi surface and form the Fermi sea below, while the single particles and excited pairs combine to describe excited electrons for $k>k_{F}$ and excited holes for $k<k_{F}$.

\section{Critical Field and Specific Heat}

The critical field for a bulk specimen of unit volume is given by

$$
H_{c}^{2} / 8 \pi=F_{n}-F_{s},
$$

where $F_{n}$ is the free energy of the normal state:

$$
\begin{aligned}
F_{n}=-4 N(0) k T \int_{0}^{\infty} d \epsilon \log (1 & \left.+e^{-\beta \epsilon}\right) \\
& =-\frac{1}{3} \pi^{2} N(0)(k T)^{2} .
\end{aligned}
$$

With the aid of (3.25) the entropy in the superconducting state, (3.15) may be expressed as

$$
T S=4 k T \sum_{k>k_{F}}\left[\ln \left(1+e^{-\beta E_{\mathbf{k}}}\right)+\beta E_{\mathrm{k}} f_{\mathrm{k}}\right] .
$$

Replacing the sum by an integral and performing a partial integration, we find

$$
T S=4 N(0) \int_{0}^{\infty} d \epsilon\left[\frac{\epsilon^{2}}{E}+E\right] f(\beta E),
$$

where the upper limit has been extended to infinity because $f(\beta E)$ decreases rapidly for $\beta \epsilon>1$. If (3.34) and (3.16) are combined with the distribution functions (3.19) and (3.25), the free energy becomes

$$
\begin{aligned}
F_{s}=-4 N(0) \int_{0}^{\infty} d \epsilon & E f(\beta E) \\
& +2 N(0) \int_{0}^{\hbar \omega} d \epsilon\left[\epsilon-\frac{\epsilon^{2}}{E}\right]-\frac{\epsilon_{0}^{2}}{V}
\end{aligned}
$$

which with the aid of (3.27) may be expressed as

$$
\begin{aligned}
F_{s}=-2 N(0) & \int_{0}^{\infty} d \epsilon\left[\frac{2 \epsilon^{2}+\epsilon_{0}^{2}}{E}\right] f(\beta E) \\
& -N(0)(\hbar \omega)^{2}\left\{\left[1+\left(\frac{\epsilon_{0}}{\hbar \omega}\right)^{2}\right]^{\frac{1}{2}}-1\right\} .
\end{aligned}
$$

${ }^{33}$ M. J. Buckingham, Phys. Rev. 101, 1431 (1956). 
The critical field is given by combining (3.31), (3.32), and (3.37) :

$$
\begin{aligned}
\frac{H_{c}^{2}}{8 \pi}=N(0)(\hbar \omega)^{2} & \left\{\left[1+\left(\frac{\epsilon_{0}}{\hbar \omega}\right)^{2}\right]^{\frac{1}{2}}-1\right\}-\frac{\pi^{2}}{3} N(0)(k T)^{2} \\
& \times\left\{1-\beta^{2} \int_{0}^{\infty} d \epsilon\left[\frac{2 \epsilon^{2}+\epsilon_{0}^{2}}{E}\right] f(\beta E)\right\} .
\end{aligned}
$$

A plot of the critical field as a function of $\left(T / T_{c}\right)^{2}$ is given in Fig. 2. The curve agrees fairly well with the $1-\left(T / T_{c}\right)^{2}$ law of the Gorter-Casimir two-fluid model, ${ }^{19}$ the maximum deviation being about four percent. There is good experimental support for a similar deviation in vanadium, thallium, indium, and tin; however, our deviation appears to be somewhat too large to fit the experimental results.

The critical field at $T=0$ is

$$
H_{0}=[4 \pi N(0)]^{\frac{1}{2}} \epsilon_{0}(0)=1.75[4 \pi N(0)]^{\frac{1}{2}} k T_{c},
$$

where $2 \epsilon_{0}(0)$ is the energy gap at $T=0$ and the density of Bloch states $N(0)$ is taken for a system of unit volume.

A law of corresponding states follows from (3.39) and may be expressed as

$$
\gamma T_{c}^{2} / H_{0}^{2}=\frac{1}{6} \pi\left[k T_{c} / \epsilon_{0}(0)\right]^{2}=0.170,
$$

where the electronic specific heat in the normal state is given by

$$
C_{e n}=\gamma T\left(\mathrm{ergs} /{ }^{\circ} \mathrm{C} \mathrm{cm}^{3}\right) \text {, }
$$

and

$$
\gamma=\frac{2}{3} \pi^{2} N(0) k^{2} \text {. }
$$

The Gorter-Casimir model gives the value of 0.159 for the ratio (3.40). The scatter of experimental data is too great to choose one value over the other at the present time.

Near $T=0$, the gap is practically independent of temperature and large compared to $k T$, and hence for

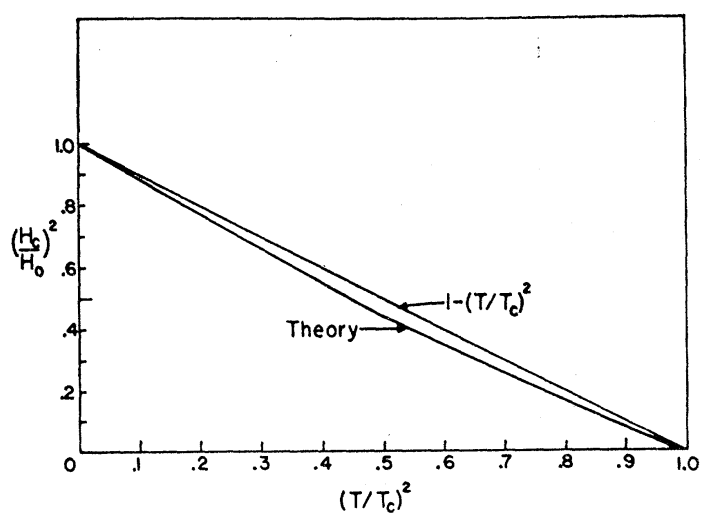

FIg. 2. Ratio of the critical field to its value at $T=0^{\circ} \mathrm{K}$ vs $\left(T / T_{c}\right)^{2}$. The upper curve is the $1-\left(T / T_{c}\right)^{2}$ law of the GorterCasimir theory and the lower curve is the law predicted by the theory in the weak-coupling limit. Experimental values generally lie between the two curves.
$T / T_{c} \ll 1$ we have the relation

or

$$
H_{c}^{2}=H_{0}^{2}\left[1-\frac{2}{3} \pi^{2}\left(k T / \epsilon_{0}\right)^{2}\right]
$$

$$
H_{c} \cong H_{0}\left[1-1.07\left(T / T_{c}\right)^{2}\right] \text {. }
$$

This approximation corresponds to neglecting the freeenergy change of the superconducting state, the total effect coming from $F_{n}$.

The electronic specific heat is most readily obtained from the entropy, (3.34):

$$
\begin{gathered}
C_{e s}=T \frac{d S}{d T}=-\beta \frac{d S}{d \beta}=-4 k \beta \sum_{k>k F} \beta E_{\mathrm{k}} \frac{d f_{\mathrm{k}}}{d \beta} \\
C_{e s}=4 k \beta^{2} \sum_{k>k_{F}} f_{\mathrm{k}}\left(1-f_{\mathrm{k}}\right)\left[E_{\mathrm{k}}{ }^{2}+\frac{\beta}{2} \frac{d \epsilon_{0}^{2}}{d \beta}\right] .
\end{gathered}
$$

The expression for $C_{e s}$ is simply interpreted as the specific heat due to electrons and holes with the modified spectrum (3.21) plus the change in condensation energy with temperature.

At the transition temperature, the energy gap vanishes and the jump in specific heat associated with the second order transition is given by

$$
\begin{array}{r}
\left(C_{e s}-C_{e n}\right) \mid T_{c}=2 k \beta^{3} \sum_{k>k F} f_{\mathrm{k} n}\left(1-f_{\mathrm{k} n}\right)\left[\frac{d \epsilon_{0}^{2}}{d \beta}\right]_{T_{c}} \\
=k N(0) \beta_{c}{ }^{2}\left[\frac{d \epsilon_{0}{ }^{2}}{d \beta}\right]_{T_{c}}
\end{array}
$$

where

$$
f_{\mathrm{k} n}=1 /\left(e^{\beta \epsilon_{\mathrm{k}}}+1\right) \text {. }
$$

The derivative $d \epsilon_{0}{ }^{2} / d \beta$ can be obtained from the relation between $\epsilon_{0}$ and $T,(3.27)$. After some calculation we find

$$
\left.\frac{d \epsilon_{0}^{2}}{d \beta}\right|_{T_{c}}=\frac{10.2}{\beta_{c}{ }^{3}}
$$

and the jump in specific heat becomes

$$
\left.\frac{C_{e s}-\gamma T_{c}}{\gamma T_{c}}\right|_{T_{c}}=1.52
$$

The Gorter-Casimir model gives 2.00 and the Koppe theory ${ }^{9}$ gives 1.71 for this ratio. The experimental data in general range between our value and 2.00 .

The initial slope of the critical-field curve at the transition temperature is given by the thermodynamic relation

$$
\left.\frac{T_{c}}{4 \pi}\left(\frac{d H_{c}}{d T}\right)^{2}\right|_{T_{c}}=\left.\left(C_{s}-C_{n}\right)\right|_{T_{c}}
$$

With use of (3.47) this becomes

$$
\left.\frac{1}{\gamma}\left(\frac{d H_{c}}{d T}\right)^{2}\right|_{T_{c}}=19.4
$$




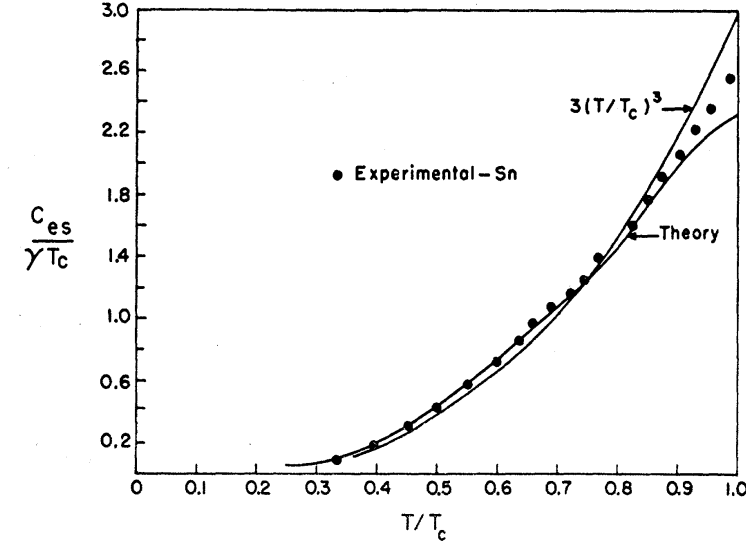

FIG. 3. Ratio of the electronic specific heat to its value in the normal state at $T_{c}$ vs $T / T_{c}$ for the Gorter-Casimir theory and for the present theory. Experimental values for tin are shown for comparison. Note added in proof.-The plotted theoretical curve is incorrect very near $T_{c}$; the intercept at $T_{c}$ should be 2.52 .

or with (3.39),

$$
\left.\frac{d H_{c}}{d T}\right|_{T_{c}}=-\frac{1.82 H_{0}}{T_{c}} .
$$

When $\beta \epsilon_{0} \gg 1$, the specific heat can be expressed in the form

$$
\begin{aligned}
\frac{C_{e s}}{\gamma T_{c}}=\frac{3}{2 \pi^{2}}\left(\frac{\epsilon_{0}}{k T_{c}}\right)^{3}\left(\frac{T_{c}}{T}\right)^{2}\left[3 K_{1}\left(\beta \epsilon_{0}\right)+K_{3}\left(\beta \epsilon_{0}\right)\right] \\
\cong 8.5 e^{-1.44 T_{c} / T},
\end{aligned}
$$

where $K_{n}$ is the modified Bessel function of the second kind.

The ratio $C_{e s} /\left(\gamma T_{c}\right)$ is plotted in Fig. 3 from (3.46) and compared with the $T^{3}$ law and the experimental values for tin. The agreement is rather good except near $T_{c}$ where our specific heat is somewhat too small. The logarithm of the same ratio is plotted in Fig. 4 to bring out the experimental deviation from the $T^{3}$ law. The recent work of Goodman et al. ${ }^{20}$ shows that the data for tin and vanadium fit the law:

$$
C_{e s} /\left(\gamma T_{c}\right)=a e^{-b T_{c} / T},
$$

with high accuracy for $T_{c} / T>1.4$, where $a=9.10$ and $b=1.50$. These values are in good agreement with our results in this region, (3.54).

Thus we see that our theory predicts the thermodynamic properties of a superconductor quite accurately and in particular gives an exponential specific heat for $T / T_{c} \ll 1$ and explicitly exhibits a second-order phase transition in the absence of a magnetic field.

\section{CALCULATION OF MATRIX ELEMENTS}

There are many problems for which one would like to determine matrix elements of a single-particle scattering operator of the form $U=\sum_{j} H_{j}$, where $H_{j}$ involves only the coordinates of particle $j$. In terms of creation and destruction operators,

$$
U=\sum_{j} H_{j}=\sum_{\mathbf{k}, \mathbf{k}^{\prime}, \sigma, \sigma^{\prime}} B_{\mathbf{k} \sigma \mathbf{k}^{\prime} \sigma^{\prime}} C_{\mathbf{k}^{\prime} \sigma^{\prime}} * c_{\mathrm{k} \sigma}
$$

where

$$
B_{\mathrm{k} \sigma \mathrm{k}^{\prime} \sigma^{\prime}}=\int \psi_{\mathrm{k}^{\prime} \sigma^{\prime}} * H_{j} \psi_{\mathrm{k} \sigma} d \tau_{j}
$$

is the matrix element for scattering of a single electron from $\mathbf{k} \sigma$ to $\mathbf{k}^{\prime} \boldsymbol{\sigma}^{\prime}$. In this section we shall determine matrix elements of $U$ between two of our many-particle excited-state wave functions for a superconductor and give tables which should be useful for application to perturbation theory and transport problems. We first give a brief review of the corresponding problems for the normal state.

The matrix element of $c_{\mathrm{k}^{\prime} \sigma^{\prime}}{ }^{*} c_{\mathrm{k} \sigma}$ between two normal state configurations is zero unless the occupation numbers differ only in transfer of an electron from $\mathbf{k} \sigma$ in the initial to $\mathbf{k}^{\prime} \sigma^{\prime}$ in the final configuration, in which case it is unity. If one wishes to calculate the probability that at temperature $T$ an electron be scattered from a state of spin $\sigma$ in an element $\Delta \mathbf{k}$ to one of spin $\sigma^{\prime}$ in $\Delta \mathbf{k}^{\prime}$, one must multiply the usual single-particle expression by $f\left(1-f^{\prime}\right)$, the probability that $\mathbf{k} \sigma$ be occupied and $\mathbf{k}^{\prime} \sigma^{\prime}$ unoccupied in a typical initial configuration. A similar factor occurs in the second-order perturbation theory expansion of $U$ :

$$
\sum_{\mathrm{k}, \sigma, \mathrm{k}^{\prime}, \sigma^{\prime}} \frac{\left|B_{\mathrm{k} \sigma \mathrm{k}^{\prime} \sigma^{\prime}}\right|^{2} f\left(1-f^{\prime}\right)}{\epsilon-\epsilon^{\prime}} .
$$

If $H_{j}$ is independent of spin, $\sigma^{\prime}=\sigma$ and the sum reduces to

$$
2 \sum_{\mathbf{k}, \mathbf{k}^{\prime}} \frac{\left|B_{\mathrm{kk}^{\prime}}\right|^{2} f\left(1-f^{\prime}\right)}{\epsilon-\epsilon^{\prime}}=-\sum_{\mathbf{k}, \mathbf{k}^{\prime}}\left|B_{\mathbf{k k}^{\prime}}\right|^{2}\left(\frac{f^{\prime}-f}{\epsilon-\epsilon^{\prime}}\right) .
$$

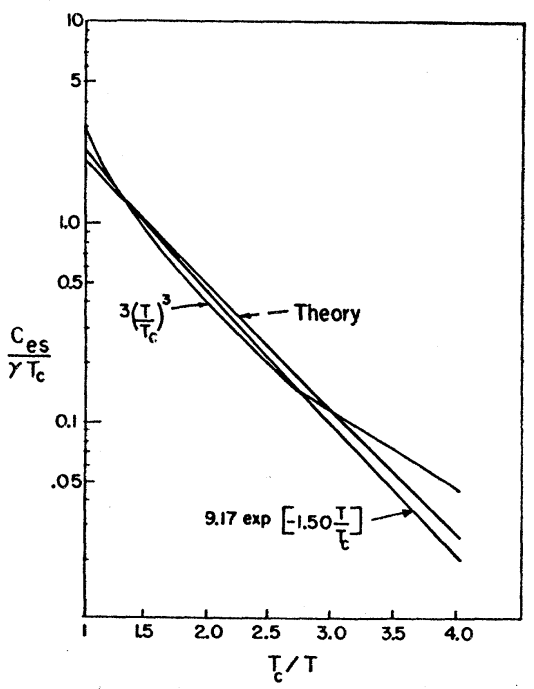

FIg. 4. A logarithmic plot of the ratio of the electronic specific heat to its value in the normal state at $T_{c} v s T_{c} / T$. The simple exponential fits the experimental data for tin and vanadium well for $T_{c} / T>1.4$ 
TABLE II. Matrix elements of single-particle scattering operator.

\begin{tabular}{|c|c|c|c|c|c|c|c|c|c|}
\hline \multicolumn{4}{|c|}{ Wave functions $\mathrm{s}^{\mathrm{a}}$} & & \multirow{3}{*}{$\begin{array}{c}\text { Energy } \\
\text { difference } \\
W_{i}-W_{f}\end{array}$} & \multirow[b]{3}{*}{$\begin{array}{l}\text { Probability of } \\
\text { initial state }\end{array}$} & \multirow{2}{*}{\multicolumn{2}{|c|}{ Matrix elements }} \\
\hline \multirow{2}{*}{\multicolumn{2}{|c|}{$\begin{array}{l}\text { Initial, } \Psi_{i} \\
\left(\mathbf{k} \uparrow, \quad\left(\mathbf{k}^{\prime} \uparrow,\right.\right. \\
\left.-\mathbf{k} \downarrow),-\mathbf{k}^{\prime} \downarrow\right)\end{array}$}} & \multirow{2}{*}{\multicolumn{2}{|c|}{$\begin{array}{c}\text { Final, } \Psi_{\boldsymbol{f}} \\
\left(\mathbf{k} \uparrow, \quad\left(\mathbf{k}^{\prime} \uparrow,\right.\right. \\
\left.-\mathbf{k} \downarrow),-\mathbf{k}^{\prime} \downarrow\right)\end{array}$}} & & & & & & \\
\hline & & & & \multicolumn{2}{|c|}{ or excited $(-)$} & & & $\begin{array}{c}c_{\mathrm{k}^{\prime} \uparrow}^{*} c_{\mathrm{k} \uparrow} \text { or } \\
c_{-\mathrm{k}^{\prime} \downarrow} c^{*} c_{\mathrm{k} \uparrow}\end{array}$ & $\begin{array}{c}c_{-\mathrm{k} \downarrow}{ }^{*} c_{-\mathrm{k}^{\prime} \downarrow} \text { or } \\
-c_{-\mathrm{k} \downarrow}{ }^{*} c_{\mathrm{k}^{\prime} \uparrow}\end{array}$ \\
\hline & & & & + & + & $E-E^{\prime}$ & $\frac{1}{2} s\left(1-s^{\prime}-p^{\prime}\right)$ & {$\left[(1-h)\left(1-h^{\prime}\right)\right]^{z}$} & $-\left(h h^{\prime}\right)^{\frac{1}{2}}$ \\
\hline$x 0$ & 00 & 00 & $X 0$ & - & - & $E^{\prime}-E$ & $\frac{1}{2} s p^{\prime}$ & $\left(h h^{\prime}\right)^{\frac{3}{3}}$ & $-\left[(1-h)\left(1-h^{\prime}\right)\right]^{z}$ \\
\hline$X 0$ & $X X$ & $X X$ & $x 0$ & + & - & $E+E$ & $\frac{1}{2} s p^{\prime}$ & $-\left[(1-h) h^{\prime}\right]^{\frac{1}{2}}$ & $-\left[h\left(1-h^{\prime}\right)\right]^{3}$ \\
\hline & & & & - & + & $-\left(E+E^{\prime}\right)$ & $\frac{1}{2} s\left(1-s^{\prime}-p^{\prime}\right)$ & $-\left[h\left(1-h^{\prime}\right)\right]^{\prime}$ & $-\left[(1-h) h^{\prime}\right]^{\frac{1}{2}}$ \\
\hline & & & & + & + & $E^{\prime}-E$ & $\frac{1}{2} s^{\prime}(1-s-p)$ & $\left(h h^{\prime}\right)^{\frac{1}{2}}$ & $-\left[(1-h)\left(1-h^{\prime}\right)\right]^{\prime}$ \\
\hline$X X$ & $0 X$ & $0 X$ & $X X$ & - & - & $E-E^{\prime}$ & $\frac{1}{2} s^{\prime} p$ & {$\left[(1-h)\left(1-h^{\prime}\right)\right]^{\frac{1}{3}}$} & $-\left(h h^{\prime}\right)^{\frac{1}{2}}$ \\
\hline 00 & $0 X$ & $0 X$ & 00 & + & - & $-\left(E+E^{\prime}\right)$ & $\frac{1}{2} s^{\prime}(1-s-p)$ & {$\left[h\left(1-h^{\prime}\right)\right]^{z}$} & {$\left[h^{\prime}(1-h)\right]^{\frac{1}{2}}$} \\
\hline & & & & - & + & $E+E^{\prime}$ & $\frac{1}{2} s^{\prime} p$ & {$\left[(1-h) h^{\prime}\right]^{k}$} & {$\left[h\left(1-h^{\prime}\right)\right]^{\prime}$} \\
\hline & & & & + & + & $E+E^{\prime}$ & $\frac{1}{4} s s^{\prime}$ & {$\left[(1-h) h^{\prime}\right]^{z}$} & {$\left[h\left(1-h^{\prime}\right)\right]^{z}$} \\
\hline$X 0$ & $0 X$ & 00 & $X X$ & - & - & $-\left(E+E^{\prime}\right)$ & $\frac{1}{4} s s^{\prime}$ & $-\left[h\left(1-h^{\prime}\right)\right]^{\frac{1}{3}}$ & $-\left[h^{\prime}(1-h)\right]^{2}$ \\
\hline & & $X X$ & 00 & + & - & $E-E^{\prime}$ & $\frac{1}{4} s s^{\prime}$ & {$\left[(1-h)\left(1-h^{\prime}\right)\right]^{h}$} & $-\left(h h^{\prime}\right)^{2}$ \\
\hline & & & & - & + & $E^{\prime}-E$ & $\frac{1}{4} s s^{\prime}$ & $-\left(h h^{\prime}\right)^{\frac{1}{2}}$ & {$\left[(1-h)\left(1-h^{\prime}\right)\right]^{k}$} \\
\hline & & & & + & + & $-\left(E+E^{\prime}\right)$ & $(1-s-p)\left(1-s^{\prime}-p^{\prime}\right)$ & {$\left[h\left(1-h^{\prime}\right)\right]^{k}$} & {$\left[(1-h) h^{\prime}\right]^{k}$} \\
\hline$X X$ & 00 & $0 X$ & $X 0$ & - & - & $E+E^{\prime}$ & $p p^{\prime}$ & $-\left[(1-h) h^{\prime}\right]^{3}$ & $-\left[h\left(1-h^{\prime}\right)\right]^{3}$ \\
\hline 00 & $X X$ & & & + & - & $E^{\prime}-E$ & $(1-s-p) p^{\prime}$ & $-\left(h h^{\prime}\right)^{\frac{3}{2}}$ & {$\left[(1-h)\left(1-h^{\prime}\right)\right]^{3}$} \\
\hline & & & & - & + & $E-E^{\prime}$ & $p\left(1-s^{\prime}-p^{\prime}\right)$ & {$\left[(1-h)\left(1-h^{\prime}\right)\right]^{\frac{1}{2}}$} & $-\left(h h^{\prime}\right)^{\frac{1}{2}}$ \\
\hline
\end{tabular}

a For transitions which change spin, reverse designations of $\left(\mathbf{k}^{\prime} \uparrow,-\mathbf{k}^{\prime} \downarrow\right)$ in the initial and in the final states.

The factor of two comes from the sum over spins in the initial configuration and the second form from the fact that $\left|B_{\mathbf{k k}^{\prime}}\right|^{2}$ is symmetric in $\mathbf{k}$ and $\mathbf{k}^{\prime}$.

The calculation of the corresponding factors for the superconducting case is complicated by the fact that any given state $\mathbf{k} \sigma$ may be occupied singly or by either ground or excited pairs, and these possibilities must be weighted by the probability that they occur in a typical initial wave function. First consider matrix elements of an operator which does not give a spin change :

$$
\left(\Psi_{f}\left|\sum_{\mathrm{k}, \mathrm{k}^{\prime}, \sigma} B_{\mathrm{kk}^{\prime}} c_{\mathrm{k}^{\prime} \sigma}{ }^{*} c_{\mathrm{k} \sigma}\right| \Psi_{i}\right)
$$

Nonvanishing matrix elements of $c_{\mathrm{k}^{\prime} \uparrow} \uparrow^{*} c_{\mathrm{k}} \uparrow$ are obtained only when the single and excited-pair occupancy of $\Psi_{i}$ and $\Psi_{f}$ is the same except for those designated by wave vectors $\mathbf{k}$ and $\mathbf{k}^{\prime}$. Further, $\Psi_{i}$ must contain a configuration in which $\mathbf{k} \uparrow$ is occupied and $\mathbf{k}^{\prime} \uparrow$ unoccupied and $\Psi_{f}$ one in which $\mathbf{k}^{\prime} \uparrow$ is occupied and $\mathbf{k} \uparrow$ unoccupied. The various possible transitions along with the matrix elements are listed in Table II. While the individual matrix elements are complicated, fairly simple results are obtained when a sum is made over all transitions in which $\mathbf{k}$ is in a volume element $\Delta \mathbf{k}$ and $\mathbf{k}^{\prime}$ in $\Delta \mathbf{k}^{\prime}$, it being assumed that $B_{\mathbf{k k}^{\prime}}$ is a continuous function of $\mathbf{k}$ and $\mathbf{k}^{\prime}$.

The first type of transition, (a), listed in Table II corresponds to single occupancy of $\mathbf{k} \uparrow$ in the initial and of $\mathbf{k}^{\prime} \uparrow$ in the final state. Pair occupancy of $\mathbf{k}^{\prime}$ (i.e., the pair $\left.\mathbf{k}^{\prime} \uparrow,-\mathbf{k}^{\prime} \downarrow\right)$ in $\Psi_{i}$ and of $\mathbf{k}$ in $\Psi_{f}$ may be either excited or ground, giving the four possible combinations listed in the second column. These have components in which the pair states $\mathbf{k}^{\prime}$ in $\Psi_{i}$ and $\mathbf{k}$ in $\Psi_{f}$ are unoccupied, designated by $X 000$ for $\Psi_{i}$ and $00 X 0$ for $\Psi_{f}$. There is a nonvanishing matrix element of $c_{\mathrm{k}^{\prime}} \uparrow{ }^{*} c_{\mathrm{k}} \uparrow$ between these components. Other components of the same wave functions have the pair states $\mathbf{k}^{\prime}$ in $\Psi_{i}$ and $\mathbf{k}$ in $\Psi_{f}$ occupied, as is indicated by the designations $X 0 X X$ and $X X X 0$, respectively. While the matrix element of $c_{k^{\prime}} \uparrow * c_{k} \uparrow$ between these latter vanishes, that of $c_{-k \downarrow} * c_{-k^{\prime} \downarrow}$ does not. Since both $c_{\mathrm{k}^{\prime}}{ }^{*} c_{\mathrm{k}} \uparrow$ and $c_{-\mathrm{k} \downarrow}{ }^{*} c_{-\mathrm{k}^{\prime} \downarrow}$ are included in the sum in (4.5), they will give coherent contributions and must be considered together. Matrix elements between these states of all other terms in the sum are zero.

Matrix elements of type (b) are for single-particle occupancy of $-\mathbf{k}^{\prime} \downarrow$ in $\Psi_{i}$ and of $-\mathbf{k} \downarrow$ in $\Psi_{f}$, while $\mathbf{k}$ in $\Psi_{i}$ and $\mathbf{k}^{\prime}$ in $\Psi_{f}$ may be occupied by either excited or ground pairs. With interchange of spin and of $\mathbf{k}$ and $\mathbf{k}^{\prime}$, they are similar to type (a). Type (c) represents single occupancy of $\mathbf{k} \uparrow$ and $-\mathbf{k}^{\prime} \downarrow$ in $\Psi_{i}$ and either excited or ground pair occupancy of both $\mathbf{k}$ and $\mathbf{k}^{\prime}$ in $\Psi_{f}$. Transitions of $c_{\mathrm{k}^{\prime} \uparrow} * c_{\mathrm{k}} \uparrow$ are allowed for the component $00 X X$ of $\Psi_{f}$ and of $c_{-\mathrm{k} \downarrow}{ }^{*} c_{-\mathrm{k}^{\prime} \downarrow}$ for the component $X X 00$. Again, these are coherent. Finally, type (d) represents excited or ground pair occupancy of $\Psi_{i}$ and single particle occupancy of $-\mathbf{k} \downarrow, \mathbf{k}^{\prime} \uparrow$ in $\Psi_{f}$.

The energy differences $W_{i}-W_{f}$ listed in the third column are obtained by taking an energy $2 E$ for an excited pair, $E$ for single occupancy, and zero for a ground pair. We have used the notation $E=E(\mathbf{k})$; $E^{\prime}=E\left(\mathbf{k}^{\prime}\right)$, etc.

In column 4 are given the probabilities of the initial state designations, based on taking $\frac{1}{2} s$ for a specified 
single occupancy, $p$ for an excited pair, and $(1-s-p)$ for a ground pair. For example, $\Psi_{i}$ in the top row corresponds to $\mathbf{k} \uparrow$ occupied and a ground pair in $\mathbf{k}^{\prime}$, and the fraction of the states $\mathbf{k}$ in the volume element $\Delta \mathbf{k}$ and $\mathbf{k}^{\prime}$ in $\Delta \mathbf{k}^{\prime}$ which have this designation is $\frac{1}{2} s(\mathbf{k})\left[1-s\left(\mathbf{k}^{\prime}\right)-p\left(\mathbf{k}^{\prime}\right)\right]$.

To calculate the matrix elements, it is convenient to decompose the wave functions into components corresponding to definite occupancy of excited and ground pairs as in (3.10). Thus, for the top row in which $\mathbf{k}^{\prime}$ in $\Psi_{i}$ and $\mathbf{k}$ in $\Psi_{f}$ are both ground pairs,

$$
\begin{aligned}
& \Psi_{i}=c_{\mathrm{k}^{\mathrm{k}}} \uparrow^{*}\left[h^{\prime \frac{1}{2}} \varphi_{01}\left(0_{\mathrm{k}}, 1_{\mathrm{k}^{\prime}}\right)+\left(1-h^{\prime}\right)^{\frac{1}{2}} \varphi_{00}\left(0_{\mathrm{k}}, 0_{\mathrm{k}^{\prime}}\right)\right], \\
& \Psi_{f}=c_{\mathrm{k}^{\prime}} \uparrow^{*}\left[h^{\frac{1}{2}} \varphi_{10}\left(1_{\mathrm{k}}, 0_{\mathrm{k}^{\prime}}\right)+(1-h)^{\frac{1}{2}} \varphi_{00}\left(0_{\mathrm{k}}, 0_{\mathrm{k}^{\prime}}\right)\right],
\end{aligned}
$$

where

$$
\varphi_{10}=b_{\mathrm{k}}{ }^{*} b_{\mathrm{k}^{\prime}} \varphi_{01} \text {. }
$$

The matrix element of $c_{\mathrm{k}^{\prime}} *^{*} c_{\mathrm{k}} \uparrow$ is

$$
\begin{aligned}
\left(\Psi_{f}\left|c_{\mathrm{k}^{\prime}} \uparrow^{*} c_{\mathrm{k}_{\mathrm{k}} \uparrow}\right| \Psi_{i}\right) & =\left[(1-h)\left(1-h^{\prime}\right)\right]^{\frac{1}{2}}\left(c_{\mathrm{k}^{\prime}}{ }^{*} \varphi_{00} \mid c_{\mathrm{k}^{\prime}} \uparrow^{*} \varphi_{00}\right) \\
& =\left[(1-h)\left(1-h^{\prime}\right)\right]^{\frac{1}{2}} .
\end{aligned}
$$

The matrix element of $c_{-\mathrm{k} \downarrow}{ }^{*} c_{-\mathrm{k}^{\prime} \downarrow}$ is given by

$$
\begin{aligned}
& \left(\Psi_{f}\left|c_{-\mathrm{k} \downarrow} * c_{-\mathrm{k}^{\prime} \downarrow}\right| \Psi_{i}\right) \\
& \quad=\left(h h^{\prime}\right)^{\frac{1}{2}}\left(c_{\mathrm{k}^{\prime}} * b_{\mathbf{k}^{\mathrm{k}}} * b_{\mathrm{k}^{\prime}} \varphi_{01} \mid c_{-\mathrm{k} \downarrow} * c_{-\mathrm{k}^{\prime} \downarrow} c_{\mathrm{k}} \uparrow^{*} \varphi_{01}\right) .
\end{aligned}
$$

Since

$$
\begin{aligned}
c_{\mathrm{k}^{\prime}}{ }^{*} b_{\mathrm{k}}{ }^{*} b_{\mathrm{k}^{\prime}} \varphi_{01} & =-c_{-\mathrm{k}^{\prime} \downarrow} b_{\mathrm{k}} * \varphi_{01}, \\
c_{-\mathrm{k} \downarrow}{ }^{*} c_{-\mathrm{k}^{\prime} \downarrow} c_{\mathrm{k}} \uparrow^{*} \varphi_{01} & =c_{-\mathrm{k}^{\prime} \downarrow} b_{\mathrm{k}}{ }^{*} \varphi_{01},
\end{aligned}
$$

the matrix element is $-\left(h h^{\prime}\right)^{\frac{1}{2}}$. The other matrix elements of types (a) and (b) may be calculated in a similar manner.

For types (c) and (d) we make use of the decomposition (3.10). For example, for type (d),

$$
\begin{aligned}
& \Psi_{i}=\alpha_{11} \varphi_{11}\left(1_{\mathrm{k}}, 1_{\mathrm{k}^{\prime}}\right)+\alpha_{10} \varphi_{10}\left(1_{\mathrm{k}}, 0_{\mathrm{k}^{\prime}}\right) \\
& +\alpha_{01} \varphi_{01}\left(0_{\mathrm{k}}, 1_{\mathrm{k}^{\prime}}\right)+\alpha_{00} \varphi_{00}\left(0_{\mathrm{k}}, 0_{\mathrm{k}^{\prime}}\right),
\end{aligned}
$$

where the $\alpha$ 's are as listed in Table I. The final wave function is

$$
\Psi_{f}=c_{\mathrm{k}^{\prime}}{ }^{*} c_{\mathrm{k}} \uparrow \varphi_{10} .
$$

Thus the matrix element of $c_{\mathrm{k}^{\prime}} *^{*} c_{\mathrm{k}} \uparrow$ is just $\alpha_{10}$. The matrix element of $c_{-\mathrm{k} \downarrow}{ }^{*} c_{-\mathrm{k}^{\prime} \downarrow}$ is found from

$$
\begin{aligned}
c_{-\mathrm{k} \downarrow} * c_{-\mathrm{k}^{\prime} \downarrow} \varphi_{01} & =c_{-\mathrm{k} \downarrow} * c_{-\mathrm{k}^{\prime} \downarrow} b_{\mathrm{k}^{\prime}} * b_{\mathrm{k}} \varphi_{10} \\
& =c_{\mathrm{k}^{\prime} \uparrow} * c_{\mathrm{k}^{\mathrm{k}} \uparrow \varphi_{10}},
\end{aligned}
$$

so that we find

$$
\left(\Psi_{f}\left|c_{-\mathrm{k} \downarrow}{ }^{*} c_{-\mathrm{k}^{\prime} \downarrow}\right| \Psi_{i}\right)=\alpha_{01} .
$$

Those for type (c) can be obtained by interchanging initial and final states and spin up and spin down.

We have so far assumed a spin-independent interaction. One involving a spin flip may be treated by exactly similar methods. Initial and final states differ from the parallel spin case by interchange of spin designation of $\mathbf{k}^{\prime}$ in the initial and in the final state. There is a coherence between the matrix elements for $c_{-\mathrm{k}^{\prime} \downarrow}{ }^{*} c_{\mathrm{k} \uparrow}$ and $c_{-\mathrm{k} \downarrow}{ }^{*} c_{\mathrm{k}^{\prime}} \uparrow$. They are the same as the corresponding matrix elements for parallel spin, except for a reversal of sign of the reverse spin transitions. For example, for the type (a) transition of the top row, the final state is now

$$
\Psi_{f}=c_{-\mathrm{k}^{\prime} \downarrow} *\left[h^{\frac{1}{2}} \varphi_{10}\left(1_{\mathrm{k}}, 0_{\mathrm{k}^{\prime}}\right)+(1-h)^{\frac{1}{2}} \varphi_{00}\left(0_{\mathrm{k}}, 0_{\mathrm{k}^{\prime}}\right)\right] .
$$

The matrix element of $c_{-\mathrm{k}^{\prime} \downarrow}{ }^{*} c_{\mathrm{k}} \uparrow$ is $\left[(1-h)\left(1-h^{\prime}\right)\right]^{\frac{1}{2}}$ as before. To obtain matrix element of $c_{-\mathrm{k} \downarrow} \downarrow^{*} c_{\mathrm{k}^{\prime}} \uparrow$, we now have, corresponding to (4.9) and (4.10),

$$
\begin{aligned}
& c_{-\mathrm{k}^{\prime} \downarrow} * b_{\mathrm{k}^{\prime}} b_{\mathrm{k}} * \varphi_{01}=c_{\mathrm{k}^{\prime} \uparrow} b_{\mathrm{k}^{2}} * \varphi_{01}, \\
& c_{-\mathrm{k} \downarrow} c_{\mathrm{k}^{\prime} \uparrow} \uparrow c_{\mathrm{k}} \uparrow^{*} \varphi_{01}=c_{\mathrm{k}^{\prime}} \uparrow b_{\mathrm{k}} * \varphi_{01},
\end{aligned}
$$

giving $+\left(h h^{\prime}\right)^{\frac{1}{2}}$. We have indicated the change in sign in the table by listing $-c_{-\mathrm{k} \downarrow} * c_{\mathrm{k}^{\prime}} \uparrow$ at the top of column 6 .

In a second-order perturbation theory calculation, one is interested in determining

$$
\sum_{f} \frac{\left|\left(\Psi_{f}\left|\sum_{\mathbf{k}, \mathbf{k}^{\prime}, \sigma} B_{\mathbf{k k}^{\prime}} c_{\mathbf{k}^{\prime} \sigma} *^{*} c_{\mathbf{k} \sigma}\right| \Psi_{i}\right)\right|^{2}}{W_{i}-W_{f}},
$$

where the sum is over all intermediate states, $f$. The initial state should be a typical one for a given temperature $T$. In general, one might have either

or

$$
B_{\mathrm{kk}^{\prime}}=+B_{-\mathrm{k}^{\prime},-\mathrm{k}}, \quad(\text { case I) }
$$

$$
B_{\mathrm{kk}^{\prime}}=-B_{-\mathrm{k}^{\prime},-\mathrm{k} .} \quad \text { (case II) }
$$

The latter applies to the magnetic interaction. To take the coherence into account, one may take the spinindependent sum over $\mathbf{k}$ and $\mathbf{k}^{\prime}$, which designate initial and intermediate states:

$$
+\sum_{\mathrm{k}, \mathrm{k}^{\prime}} \frac{\left|B_{\mathrm{kk}^{\prime}}\right|^{2}\left\langle\left|\left(\Psi_{f}\left|c_{\mathrm{k}^{\prime} \uparrow}{ }^{*} c_{\mathrm{k} \uparrow} \pm c_{-\mathrm{k} \downarrow} * c_{-\mathrm{k}^{\prime} \downarrow}\right| \Psi_{i}\right)\right|^{2}\right\rangle_{\mathrm{Av}}}{W_{i}-W_{f}}
$$

where the average is taken over volume elements $\Delta \mathbf{k}$ and $\Delta \mathbf{k}^{\prime}$ for the initial state.

For terms with an energy denominator $W_{i}-W_{f}$ $=E-E^{\prime}$, we have

$$
\begin{aligned}
\left\{\left[(1-h)\left(1-h^{\prime}\right)\right]^{\frac{1}{3} \mp}(\right. & \left.\left(h h^{\prime}\right)^{\frac{1}{2}}\right\}^{2}\left[\frac{1}{2} s\left(1-s^{\prime}-p^{\prime}\right)\right. \\
& \left.+\frac{1}{2} p s^{\prime}+\frac{1}{4} s s^{\prime}+p\left(1-s^{\prime}-p^{\prime}\right)\right] \\
& =\frac{1}{2}\left\{1+\frac{\epsilon \epsilon^{\prime} \mp \epsilon_{0}^{2}}{E E^{\prime}}\right\} f\left(1-f^{\prime}\right) .
\end{aligned}
$$

Table III lists the average matrix elements for the various values of $W_{i}-W_{f}$.

The second-order perturbation theory sum may be written

$$
-\sum_{\mathbf{k}, \mathbf{k}^{\prime}}\left|B_{\mathbf{k k}^{\prime}}\right|^{2} L\left(\epsilon, \epsilon^{\prime}\right)
$$


TABLE III. Mean square matrix elements for possible values of $W_{i}-W_{f}$.

\begin{tabular}{|c|c|}
\hline$W_{i}-W_{f}$ & $\left\langle\left|\left(\Psi_{f}\left|c_{\mathrm{k}^{\prime} \uparrow^{*}} c_{\mathrm{k} \uparrow} \pm c_{-\mathrm{k} \downarrow}{ }^{*} c_{-\mathrm{k}^{\prime} \downarrow}\right| \Psi_{i}\right)\right|^{2}\right\rangle_{\mathrm{Av}}$ \\
\hline$E-E^{\prime}$ & $\frac{1}{2}\left(1+\frac{\epsilon \epsilon^{\prime} \mp \epsilon_{0}^{2}}{E E^{\prime}}\right) f\left(1-f^{\prime}\right)$ \\
\hline$E^{\prime}-E$ & $\frac{1}{2}\left(1+\frac{\epsilon \epsilon^{\prime} \mp \epsilon_{0}^{2}}{E E^{\prime}}\right) f^{\prime}(1-f)$ \\
\hline$-\left(E+E^{\prime}\right)$ & $\frac{1}{2}\left(1-\frac{\epsilon \epsilon^{\prime} \mp \epsilon_{0}^{2}}{E E^{\prime}}\right)(1-f)\left(1-f^{\prime}\right)$ \\
\hline$E+E^{\prime}$ & $\frac{1}{2}\left(1-\frac{\epsilon \epsilon^{\prime} \mp \epsilon_{0}^{2}}{E E^{\prime}}\right) f f^{\prime}$ \\
\hline
\end{tabular}

where

$$
\begin{aligned}
L\left(\epsilon, \epsilon^{\prime}\right)= & \frac{1}{2}\left(1+\frac{\epsilon \epsilon^{\prime} \mp \epsilon_{0}^{2}}{E E^{\prime}}\right)\left(\frac{f^{\prime}-f}{E-E^{\prime}}\right) \\
& +\frac{1}{2}\left(1-\frac{\epsilon \epsilon^{\prime} \mp \epsilon_{0}^{2}}{E E^{\prime}}\right)\left(\frac{1-f-f^{\prime}}{E+E^{\prime}}\right) \\
= & \frac{1}{2}\left(\frac{(1-2 f) E-\left(1-2 f^{\prime}\right) E^{\prime}}{\epsilon^{2}-\epsilon^{\prime 2}}\right) \\
& +\frac{1}{2}\left(\frac{\epsilon \epsilon^{\prime} \mp \epsilon_{0}^{2}}{E E^{\prime}}\right)\left(\frac{(1-2 f) E^{\prime}-\left(1-2 f^{\prime}\right) E}{\epsilon^{2}-\epsilon^{\prime 2}}\right) .
\end{aligned}
$$

The upper signs correspond to case I, the lower to case II.

To determine the probability of a transition in which an energy quantum $h \nu$ is absorbed, we have a sum of the form

$$
\begin{array}{r}
\frac{2 \pi}{\hbar} \sum_{\mathbf{k}, \mathbf{k}^{\prime}}\left|B_{\mathrm{kk}^{\prime}}\right|^{2}\left\langle\left|\left(\Psi_{f}\left|c_{\mathrm{k}^{\prime} \uparrow}{ }^{*} c_{\mathrm{k} \uparrow} \pm c_{-\mathrm{k} \downarrow} c_{-\mathrm{k}^{\prime} \downarrow}\right| \Psi_{i}\right)\right|^{2}\right\rangle_{\mathrm{Av}} \\
\times \delta\left(W_{f}-W_{i}-h \nu\right) .
\end{array}
$$

For the matrix elements for which $W_{f}-W_{i}=E-E^{\prime}$, we may interchange $\mathbf{k}$ and $\mathbf{k}^{\prime}$ in the sum and combine them with those for which $W_{f}-W_{i}=E^{\prime}-E$. This just gives either one multiplied by a factor of two:

$$
\begin{aligned}
\frac{2 \pi}{\hbar} \sum_{\mathbf{k}, \mathbf{k}^{\prime}} 2\left|B_{\mathbf{k k}^{\prime}}\right|^{2 \frac{1}{2}}(1+ & \left.\frac{\epsilon \epsilon^{\prime} \mp \epsilon_{0}{ }^{2}}{E E^{\prime}}\right) \\
& \times f\left(1-f^{\prime}\right) \delta\left(W_{f}-W_{i}-h \nu\right) .
\end{aligned}
$$

One may interpret the factor of two as accounting for the sum over the two spin possibilities of the initial state. If $\left|B_{\mathrm{k}^{\prime}}\right|^{2}$ is symmetric with respect to the Fermi surface, so that we may sum over + and - values of $\epsilon$ and $\epsilon^{\prime}$, terms odd in $\epsilon$ and $\epsilon^{\prime}$ drop out, and we find

$$
\begin{aligned}
\frac{2 \pi}{\hbar} \sum_{k, k^{\prime}>k_{F}} 4\left|B_{\mathrm{kk}}\right|^{2}(1 \mp & \left.\frac{\epsilon_{0}^{2}}{E E^{\prime}}\right) \\
& \times f\left(1-f^{\prime}\right) \delta\left(E^{\prime}-E-h \nu\right) .
\end{aligned}
$$

The corresponding expressions for $W_{f}-W_{i}=E+E^{\prime}$ and $-\left(E+E^{\prime}\right)$ are:

$$
\begin{aligned}
& \frac{2 \pi}{\hbar} \sum_{k, k^{\prime}>k_{F}} 2\left|B_{\mathrm{kk}}\right|^{2}\left(1 \pm \frac{\epsilon_{0}^{2}}{E E^{\prime}}\right) \\
& \times(1-f)\left(1-f^{\prime}\right) \delta\left(E+E^{\prime}-h \nu\right), \\
& \frac{2 \pi}{\hbar} \sum_{k, k^{\prime}>k F} 2\left|B_{\mathrm{kk}}\right|^{2}\left(1 \pm \frac{\epsilon_{0}^{2}}{E E^{\prime}}\right) f f^{\prime} \delta\left(E+E^{\prime}+h \nu\right),
\end{aligned}
$$

respectively, where again we have dropped terms odd in $\epsilon$ and $\epsilon^{\prime}$.

Hebel and Slichter ${ }^{34}$ have used (4.25) to estimate the temperature dependence of the relaxation time for nuclear spin resonance in the superconducting state from the corresponding value in the normal state. They are able to account for an observed initial decrease in relaxation time in $\mathrm{Al}$ as the temperature is lowered below $T_{c}$. The increased density of states in energy in the superconducting phase more than makes up for the decrease in number of excited electrons at temperatures not too far below $T_{c}$. For this problem, the lower sign $(+)$ is appropriate.

These expressions may also be used to determine transport properties, such as electrical conductivity in the microwave region and thermal conductivity.

Note added in proof.- The marked effect of coherence on the matrix elements is verified experimentally by comparing absorption of ultrasonic waves, which follows case I, with nuclear spin relaxation or electromagnetic absorption, both of which follow case II. For frequencies such that $h \nu \ll k T_{c}$, one expects for case II an initial increase in absorption just below $T_{c}$, followed by a decrease to values below that of the normal state as the temperature is lowered, as is observed experimentally. On the other hand, for case I one expects the absorption to drop with an infinite slope at $T_{c}$, such as is found for ultrasonic waves.

The expressions for the transition probabilities are simplified if we change our convention for the moment to give $E$ the same sign as $\epsilon$, so that $E=-\left(\epsilon^{2}+\epsilon_{0}^{2}\right)^{1}$ below the Fermi surface. One may then write (4.26) and (4.27) in the same form as (4.25), with $E$ and $E^{\prime}$ now taking on both positive and negative values. Considering both direct absorption and induced emission, the net rate of absorption of energy in the superconducting state is proportional to

$$
\alpha_{s} \propto \int\left(1 \mp \frac{\epsilon_{0}^{2}}{E E^{\prime}}\right)\left[f\left(1-f^{\prime}\right)-f^{\prime}(1-f)\right] \rho(E) \rho\left(E^{\prime}\right) d E,
$$

where $E^{\prime}=E+h \nu$ and $\rho(E)=N(0) E /\left(E^{2}-\epsilon_{0}^{2}\right)^{\frac{1}{2}}$ is the density of states in energy.

With the upper sign (case I) and with $h \nu \ll k T$, the density of states terms are cancelled by the first factor, and the expression reduces to

$$
\alpha_{s} \propto 2[N(0)]^{2} \int_{\epsilon_{0}}^{\infty}\left(f-f^{\prime}\right) d E \cong 2[N(0)]^{2} h v f\left(\epsilon_{0}\right),
$$

The factor 2 comes from adding contributions above and below the Fermi surface. The corresponding expression for the normal

${ }^{34}$ L. C. Hebel and C. P. Slichter, Phys. Rev. 107, 901 (1957). We are indebted to these authors for considerable help in working out the details of the calculation of matrix elements, particularly in regard to taking into account the coherence of matrix elements of opposite spin. 
state is similar, except that $\epsilon_{0}=0$. We thus find

$$
\alpha_{s} / \alpha_{n}=2 f\left(\epsilon_{0}\right) \text {. }
$$

R. W. Morse and H. V. Bohm (to be published) have used (4.29) for analysis of data on ultrasonic attenuation in an indium specimen for which the electronic mean free path is large compared with the wavelength of the ultrasonic wave, so that one might expect the theory to apply. Values of $\epsilon_{0}(T)$ estimated from the data by use of (4.29) are in excellent agreement with our theoretical values (Fig. 1).

For case II, the integral may be expressed in the form:

$$
\frac{\alpha_{s}}{\alpha_{n}}=\frac{1}{h \nu} \int \frac{\left(E E^{\prime}+\epsilon_{0}^{2}\right)\left(f-f^{\prime}\right) d E}{\left\{\left(E^{2}-\epsilon_{0}^{2}\right)\left[(E+h \nu)^{2}-\epsilon_{0}^{2}\right]\right\}^{3}} .
$$

The integral diverges at $E=\epsilon_{0}$ if $h \nu$ is set equal to zero in the denominator. Numerical evaluation of the integral indicates that for $h \nu \sim \frac{1}{2} k T_{c}$ or less, (4.30) gives an increase in absorption just below $T_{c}$ as observed by Hebel and Slichter in nuclear magnetic resonance and by Tinkham and co-workers (private communication) for microwave absorption in thin superconducting films.

In order to have absorption at $T=0, h \nu$ must be greater than the energy gap, $2 \epsilon_{0}$. We take $E$ negative and $E^{\prime}$ positive, and find for this case:

$$
\frac{\alpha_{s}}{\alpha_{n}}=\frac{1}{h \nu} \int_{\epsilon_{0}-h \nu}^{-\epsilon_{0}} \frac{\left[E(E+h \nu)+\epsilon_{0}^{2}\right] d E}{\left\{\left(E^{2}-\epsilon_{0}^{2}\right)\left[(E+h \nu)^{2}-\epsilon_{0}^{2}\right]\right\}^{\frac{1}{2}}} .
$$

The integral may be evaluated in terms of the complete elliptic integrals, $E(\gamma)$ and $K(\gamma)$ as follows:

$$
\frac{\alpha_{s}}{\alpha_{n}}=\left(1+\frac{2 \epsilon_{0}}{h \nu}\right) E(\gamma)-2\left(\frac{2 \epsilon_{0}}{h \nu}\right) K(\gamma),
$$

where

$$
\gamma=\left(h \nu-2 \epsilon_{0}\right) /\left(h \nu+2 \epsilon_{0}\right) .
$$

This expression is in excellent agreement with data of Glover and Tinkham (reference 20, Fig. 6) on infrared absorption in thin films.

\section{ELECTRODYNAMIC PROPERTIES}

The electrodynamic properties of our model are determined using a perturbation treatment in which the first order change in the wave function is used to calculate the current as a functional of the field. For such properties as the Meissner effect this approach is quite rigorous since we are interested in the limit as $\mathbf{A}(\mathbf{r})$ approaches zero. It is assumed that the medium is infinite and that the sources of the field may be introduced by inserting current sheets in the interior. This method has been applied previously to the calculation of the diamagnetic properties of an electron gas. ${ }^{35}$

We first derive an expression, valid for arbitrary temperatures, relating the current density to the total field (the field due to the sources and to the induced currents). The fact that the system displays a Meissner effect is established by investigating the Fourier transform of the current density in the limit that $q \rightarrow 0$. In this limit we obtain the equation,

$$
\lim _{\mathbf{q} \rightarrow 0} \mathbf{j}(\mathbf{q})=-\frac{1}{c \Lambda_{T}} \mathfrak{a}(\mathbf{q}),
$$

${ }^{35}$ This method was first applied to the calculation of the diamagnetic properties of an electron gas by O. Klein, Arkiv Mat. Astron Fysik, A31, No. 12 (1944). Our treatment follows that of one of the authors as given in reference 7, pp. 303-321, where further references to the literature may be found where $\Lambda_{T}$ is a function of temperature, increasing, in the free-electron approximation, from the London value $\Lambda=m / n e^{2}$ at $T=0$ to infinity at the transition temperature.

The limiting expression (5.1) is valid only for values of $q$ smaller than those important for most penetration phenomena. In general we find the current density is a functional of the vector potential A which, with $\operatorname{div} \mathbf{A}$ $=0$, may be expressed in a form similar to that proposed by Pippard (1.3):

$$
\mathbf{j}(\mathbf{r})=-\frac{3}{4 \pi c \Lambda_{T} \xi_{0}} \int \frac{\mathbf{R}\left[\mathbf{R} \cdot \mathbf{A}\left(\mathbf{r}^{\prime}\right)\right] J(R, T) d \mathbf{r}^{\prime}}{R^{4}} .
$$

The kernel, $J(R, T)$, is a relatively slowly varying function of temperature, and at $T=0^{\circ} \mathrm{K}$ is not far different from Pippard's $\exp \left(-R / \xi_{0}\right)$.

To calculate penetration depths, it is more convenient to use the Fourier transform of (5.2), which may be expressed in the form

$$
\mathbf{j}(\mathbf{q})=-(c / 4 \pi) K(q) \mathfrak{a}(\mathbf{q}),
$$

where $K(q)$ is a scalar which approaches the constant value $4 \pi /\left(\Lambda_{T} c^{2}\right)$ in the limit $q \rightarrow 0$. One may determine $K(q)$ directly from the perturbation expansion of the wave function, or one may first calculate $J(R, T)$ and then find the transform of (5.2). The latter procedure is followed in Appendix $\mathrm{C}$, where an explicit expression for $K(q)$ valid for $q$ not too small is derived. In this section we shall give a direct derivation of the transform which can be used to investigate the limit $q \rightarrow 0$, and then give the derivation of (5.2). A comparison of calculated and observed values of penetration depths is given at the end of the section.

In the absence of the electromagnetic field, the system at a given temperature is characterized by the complete orthonormal set of wave functions which we denote by

$$
\Psi_{0}(T), \Psi_{1}(T), \cdots \Psi_{n}(T), \cdots,
$$

with corresponding energies

$$
W_{0}(T), W_{1}(T) \cdots W_{n}(T) \cdots
$$

We choose for $\Psi_{0}(T)$ a typical wave function of the type described in the previous sections, where the occupation of "single particles" and "excited pairs" is given by the $s$ and $p$ distributions, respectively, appropriate to the temperature $T$; the rest of the phase space is available for "ground pairs" whose distribution is specified by $h$ which is also a function of $T$. The set of orthogonal states is obtained by varying $s$ and $p$ (in analogy with the normal metal) and not changing $h$. We thus are choosing a representative configuration of the most probable distribution and taking system averages with respect to this representative configuration.

The electromagnetic interaction term for an electron of charge $q=-e, e>0$, is, in second quantized form, 


$$
\begin{aligned}
H_{I}=\int d \mathbf{r} \psi^{*}(\mathbf{r})\left[\frac{-i e \hbar}{2 m c}(\mathbf{A} \cdot \boldsymbol{\nabla}\right. & +\boldsymbol{\nabla} \cdot \mathbf{A}) \\
& \left.+\frac{e^{2}}{2 m c^{2}} \mathbf{A}^{2}(\mathbf{r})\right] \psi(\mathbf{r}) .
\end{aligned}
$$

We choose a gauge in which $\boldsymbol{\nabla} \cdot \mathbf{A}=0$ and in which $\mathbf{A}=0$ if the magnetic field is zero.

We expand $\psi$ and $\psi^{*}$ in creation and annihilation operators $^{36}$ :

$$
\begin{aligned}
\psi(\mathbf{r}) & =\frac{1}{\Omega^{\frac{1}{2}}} \sum_{\mathbf{k}, \sigma} c_{\mathrm{k}, \sigma} u_{\sigma} e^{i \mathbf{k} \cdot \mathbf{r}} \\
\psi^{*}(\mathbf{r}) & =\frac{1}{\Omega^{\frac{1}{2}}} \sum_{\mathbf{k}^{\prime}, \sigma^{\prime}} c_{\mathrm{k}^{\prime}, \sigma^{\prime}} * u_{\sigma^{\prime}} * e^{-i \mathbf{k}^{\prime} \cdot \mathbf{r}}
\end{aligned}
$$

where the $c$ 's satisfy the usual fermion anticommutation relations, (2.1) and (2.2), $u_{\sigma}$ is a two-component spinor, and $\Omega$ is the volume of the container. The interaction Hamiltonian becomes, when one neglects the term of higher order in $\mathbf{A}$,

where

$$
H_{I}=\frac{e \hbar}{m c} \frac{(2 \pi)^{\frac{3}{2}}}{\Omega} \sum_{\mathbf{k}, \mathbf{q}, \sigma} c_{\mathbf{k}+\mathbf{q}, \sigma}{ }^{*} c_{\mathrm{k}, \sigma} \mathfrak{a}(\mathbf{q}) \cdot \mathbf{k}
$$

$$
\mathfrak{a}(\mathbf{q})=\left(\frac{1}{2 \pi}\right)^{\frac{3}{2}} \int d \mathbf{r} \mathbf{A}(\mathbf{r}) e^{-i \mathbf{q} \cdot \mathbf{r}}
$$

The current operator $\mathfrak{I}(\mathbf{r})$ is

$$
\begin{aligned}
\mathfrak{I}(\mathbf{r})=\frac{i e \hbar}{2 m}\left(\psi^{*} \nabla \psi-\text { Herm. conj. }\right) & -\frac{e^{2}}{m c} \psi^{*} \mathbf{A} \psi \\
& =\mathfrak{I}_{P}(\mathbf{r})+\mathfrak{I}_{D}(\mathbf{r}) .
\end{aligned}
$$

Expanding $\psi$ and $\psi^{*}$ as in (5.6), we get

$$
\begin{aligned}
& \mathfrak{I}_{P}(\mathbf{r})=\frac{e \hbar}{2 m \Omega} \sum_{\mathbf{k}, \mathbf{q}, \sigma} c_{\mathrm{k}+\mathbf{q}, \sigma}{ }^{*} c_{\mathrm{k}, \sigma} e^{-i \mathbf{q} \cdot \mathbf{r}}(2 \mathbf{k}+\mathbf{q}), \\
& \mathfrak{J}_{D}(\mathbf{r})=-\frac{e^{2}}{m c} \frac{1}{\Omega} \sum_{\mathbf{k}, \mathbf{q}, \sigma} c_{\mathbf{k}+\mathbf{q}, \sigma}{ }^{*} c_{\mathrm{k}, \sigma} e^{-i \mathbf{q} \cdot \mathbf{r}} \mathbf{A}(\mathbf{r}) .
\end{aligned}
$$

In the presence of the electromagnetic field the wave function for the system may be written

$$
\Phi(\mathbf{A})=\Phi_{0}+\Phi_{1}+\left(\text { terms of order } \mathbf{A}^{2} \cdots\right),
$$

where the usual perturbation expression for $\Phi_{1}$ is

$$
\left.\Phi_{1}=\sum_{i \neq 0} \frac{\left(\Psi_{i}\left|H_{I}\right| \Psi_{0}\right)}{W_{0}-W_{i}} \mid \Psi_{i}\right) .
$$

${ }^{36}$ At this point we insert plane waves for the Bloch functions. It would be possible to carry through an analogous procedure formally with Bloch functions. The average matrix elements which enter cannot be evaluated explicitly, but can be expressed in terms of empirically determined parameters. The appropriate modifications of the free-electron expressions are as indicated in the introduction.
To the lowest order in $\mathbf{A}(\mathbf{r})$, the expectation value of the current operator is then

$$
\begin{aligned}
\mathbf{j}(\mathbf{r})=(\Phi|\mathfrak{J}(\mathbf{r})| \Phi)=\left(\Phi_{1}\left|\mathfrak{J}_{P}(\mathbf{r})\right| \Phi_{0}\right) \\
+\left(\Phi_{0}\left|\mathfrak{\Im}_{P}(\mathbf{r})\right| \Phi_{1}\right)+\left(\Phi_{0}\left|\mathfrak{\Im}_{D}(\mathbf{r})\right| \Phi_{0}\right)
\end{aligned}
$$

where the last equality follows if the current in the field free state, $\mathbf{j}_{0}(\mathbf{r})=\left(\Phi_{0}|\mathfrak{I}(\mathbf{r})| \Phi_{0}\right)$, vanishes. ${ }^{36 \mathrm{a}}$

If (5.7), (5.9), and (5.11) are combined with (5.12) and the condition $\mathbf{q} \cdot \mathfrak{a}(\mathbf{q})=0$ is used, we obtain for the paramagnetic part of the current density,

$$
\begin{aligned}
\mathbf{j}_{P}(\mathbf{r})= & -\frac{e^{2} \hbar^{2}(2 \pi)^{\frac{3}{2}}}{2 m^{2} c \Omega^{2}} \sum_{i \neq 0} \sum_{\mathbf{k}, \mathbf{q}, \sigma} \sum_{\mathbf{k}^{\prime}, \mathbf{q}^{\prime}, \sigma^{\prime}}(2 \mathbf{k}+\mathbf{q}) \mathbf{k}^{\prime} \\
& \cdot\left[\mathfrak{a}\left(\mathbf{q}^{\prime}\right) e^{-i \mathbf{q} \cdot \mathbf{r}}\left(\Psi_{0}(T)\left|c_{\mathbf{k}^{\prime}+\mathbf{q}^{\prime}, \sigma^{\prime}}{ }^{*} c_{\mathbf{k}^{\prime} \sigma^{\prime}}\right| \Psi_{i}(T)\right)\right. \\
& \times\left(\Psi_{i}(T)\left|c_{\mathbf{k}+\mathbf{q}, \sigma}{ }^{*} c_{\mathbf{k}, \sigma}\right| \Psi_{0}(T)\right) \frac{1}{W_{0}-W_{i}} \\
& + \text { complex conjugate }]
\end{aligned}
$$

while for the diamagnetic part we have

$$
\mathbf{j}_{D}(\mathbf{r})=-\left(n e^{2} / m c\right) \mathbf{A}(\mathbf{r})
$$

where $n$ is the number of conduction electrons, of both spin directions, per unit volume.

The spin sums and the calculation of the average matrix elements can be carried out as indicated in Sec. IV, (4.16) to (4.22). We then obtain

$$
\begin{aligned}
\mathbf{j}_{P}(\mathbf{r})=\frac{e^{2} \hbar^{2}}{2 m^{2} c} \frac{(2 \pi)^{\frac{3}{2}}}{\Omega^{2}} \sum_{\mathbf{k}, \mathbf{q}}(2 \mathbf{k}+\mathbf{q}) \mathbf{k} & \\
\cdot & \mathfrak{a}(-\mathbf{q}) e^{-i \mathbf{q} \cdot \mathbf{r}} L\left(\epsilon_{\mathbf{k}}, \epsilon_{\mathbf{k}+\mathbf{q}}\right),
\end{aligned}
$$

where $L\left(\epsilon_{\mathrm{k}}, \epsilon_{\mathrm{k}+\mathrm{q}}\right)$ is given by (4.22) with the lower signs, corresponding to case II. Setting $\epsilon_{\mathrm{k}}=\epsilon$ and $\epsilon_{\mathrm{k}+\mathrm{q}}$ $=\epsilon^{\prime}$, the explicit expression for $L$ is

$$
\begin{aligned}
L\left(\epsilon, \epsilon^{\prime}\right)=\frac{1}{2}\left(\frac{1-f-f^{\prime}}{E+E^{\prime}}\right) & \left(1-\frac{\epsilon \epsilon^{\prime}+\epsilon_{0}^{2}}{E E^{\prime}}\right) \\
& +\frac{1}{2}\left(\frac{f^{\prime}-f}{E-E^{\prime}}\right)\left(1+\frac{\epsilon \epsilon^{\prime}+\epsilon_{0}^{2}}{E E^{\prime}}\right) .
\end{aligned}
$$

36a Note added in proof.-We neglect the effects of the momentum dependent cutoff on the expression for the current density; as shown explicitly by $\mathrm{P}$. W. Anderson (private communication) errors introduced are negligible in the weak coupling limit. In a general gauge, $\mathbf{A}=\mathbf{A}_{0}+\operatorname{grad} \varphi$, with $\operatorname{div} \mathbf{A}_{0}=0$, it would be necessary to include in the perturbation expansion collective excitations of the electrons, the simplest of which corresponds to a uniform displacement of all of the electrons in momentum space. Energies and matrix elements of collective excitations are nearly the same in normal and superconducting phases. We assume the collective excitations make a negligible contribution to the current form $\mathbf{A}_{0}$ (see J. Bardeen, Nuovo Cimento 5, 1766 (1957)). 
To show how (5.16) reduces to the usual expression for a free-electron gas in the limit $\epsilon_{0} \rightarrow 0$, we note that $E$ is defined so that it is intrinsically positive, while the Bloch energy $\epsilon$ may be either positive or negative. As $\epsilon_{0} \rightarrow 0, E \rightarrow|\epsilon|$. If $\epsilon$ is negative,

$$
f(\epsilon)=f(-|\epsilon|)=1-f(|\epsilon|)=1-f(E),
$$

To get a nonvanishing contribution from the first line, $\epsilon$ and $\epsilon^{\prime}$ must have opposite signs, while for the second line they must have the same signs. We thus find that $L\left(\epsilon, \epsilon^{\prime}\right) \rightarrow\left(f^{\prime}-f\right) /\left(\epsilon-\epsilon^{\prime}\right)$, as it should [see (4.4)].

\section{The Meissner Effect}

To establish the existence of the Meissner effect, we investigate the Fourier transform of $\mathbf{j}(\mathbf{r})$ in the limit $\mathbf{q} \rightarrow 0$. If $\mathbf{j}(\mathbf{q})$ does not go to zero in this limit, and is opposite in sign to $\mathfrak{a}(\mathbf{q})$, then the system will expel the field from its interior and behave like a perfect diamagnet.

The Fourier transform of the paramagnetic part of the current density, $\mathbf{j}_{P}(\mathbf{r})$, is given by

$$
\mathbf{j}_{P}(\mathbf{q})=\left(\frac{1}{2 \pi}\right)^{\frac{3}{2}} \int d \mathbf{r} \mathbf{j}_{P}(\mathbf{r}) e^{-i \mathbf{q} \cdot \mathbf{r}}
$$

Referring to (5.15), this can be written as

$$
\mathbf{j}_{P}(\mathbf{q})=\frac{e^{2} \hbar^{2}}{m^{2} c}\left(\frac{1}{2 \pi}\right)^{3} \int d \mathbf{k}(2 \mathbf{k}+\mathbf{q}) \mathbf{k} \cdot \mathfrak{a}(\mathbf{q}) L\left(\epsilon_{\mathbf{k}}, \epsilon_{\mathbf{k}+\mathbf{q}}\right),
$$

where in the limit that $\mathbf{q} \rightarrow 0, \epsilon_{\mathrm{k}}=\epsilon_{\mathrm{k}+\mathrm{q}}$ and $L\left(\epsilon_{\mathrm{k}}, \epsilon_{\mathrm{k}+\mathrm{q}}\right)$ becomes

$$
\lim _{\mathrm{q} \rightarrow 0} L\left(\epsilon_{\mathrm{k}}, \epsilon_{\mathrm{k}+\mathrm{q}}\right)=\frac{\beta e^{\beta E}}{\left(1+e^{\beta E}\right)^{2}} .
$$

Thus we want to evaluate

$$
\lim _{\mathbf{q} \rightarrow 0} \mathbf{j}_{P}(\mathbf{q})=\frac{e^{2} \hbar^{2}}{m^{2} c}\left(\frac{1}{2 \pi}\right)^{3} 2 \beta \int d \mathbf{k} \mathbf{k} \mathbf{k} \cdot \mathfrak{a}(\mathbf{q}) \frac{e^{\beta E}}{\left(1+e^{\beta E}\right)^{2}} .
$$

Choosing $\mathfrak{a}(\mathbf{q})$ as the polar axis, the angular integration can be done; then, using the relations $n=k_{F}{ }^{3} / 3 \pi^{2}$ and $\mathcal{E}_{F}=\hbar^{2} k_{F}^{2} / 2 m$, we get

$$
\lim _{\mathbf{q} \rightarrow 0} \mathbf{j}_{P}(\mathbf{q})=\frac{n e^{2}}{m c}\left(1-\frac{\Lambda}{\Lambda_{T}}\right) \mathfrak{a}(\mathbf{q})
$$

where

$$
1-\frac{\Lambda}{\Lambda_{T}} \equiv \frac{2 \beta \mathscr{E}_{F}}{k_{F}{ }^{5}} \int_{0}^{\infty} k^{4} d k e^{\beta E}\left(1+e^{\beta E}\right)^{-2}
$$

Letting $y=\epsilon / k T$, and using the sharp maximum of the integrand at $\epsilon=0$, we find

$$
1-\frac{\Lambda}{\Lambda_{T}}=2 \int_{0}^{\infty} d y \frac{\exp \left(y^{2}+\beta^{2} \epsilon_{0}^{2}\right)^{\frac{1}{2}}}{\left[1+\exp \left(y^{2}+\beta^{2} \epsilon_{0}^{2}\right)^{\frac{1}{2}}\right]^{2}} .
$$

The London constant $\Lambda_{T}$ can also be expressed in terms of derivatives of $\epsilon_{0}$, as is done in Appendix $\mathrm{C}$ where the following result is derived

$$
\Lambda_{T}=\Lambda\left(1+\frac{\beta}{\epsilon_{0}} \frac{d \epsilon_{0}}{d \beta}\right) .
$$

The total induced current thus becomes:

$\lim _{\mathbf{q} \rightarrow 0} \mathbf{j}(\mathbf{q})=\mathbf{j}_{P}+\mathbf{j}_{D}=-\frac{\Lambda}{\Lambda_{T}} \frac{n e^{2}}{m c}, \quad \mathfrak{a}(\mathbf{q})=-\frac{1}{\Lambda_{T} c} \mathfrak{a}(\mathbf{q})$.

In the two limiting situations $T \rightarrow 0$ and $T \rightarrow T_{c}, \Lambda / \Lambda_{T}$ can very easily be evaluated. As $T \rightarrow 0, \beta$ becomes infinite, so that $\Lambda_{T}$ becomes equal to $\Lambda$, the London value

$\lim _{T \rightarrow 0}\left(\Lambda / \Lambda_{T}\right)=1-2 \int_{0}^{\infty} d y \exp \left[-\left(y^{2}+\beta^{2} \epsilon_{0}^{2}\right)^{\frac{1}{2}}\right]=1$.

This could have been seen immediately from (5.19) since in the $T \rightarrow 0$ limit $L\left(\epsilon_{\mathrm{k}}, \epsilon_{\mathrm{k}+\mathrm{q}}\right)=0$ and the paramagnetic part of the current density is zero. This limit just gives the equation obtained by London assuming complete rigidity of the unperturbed system wave function in the presence of an electromagnetic field. When $T \rightarrow T_{c}, \epsilon_{0} \beta$ goes to zero and $\Lambda / \Lambda_{T}$ also goes to zero, since

$$
\lim _{T \rightarrow T_{c}}\left(\frac{\Lambda}{\Lambda_{T}}\right)=1-2 \int_{0}^{\infty} \frac{e^{y} d y}{\left(1+e^{y}\right)^{2}}=0 .
$$

The small Landau diamagnetism would appear only in a higher order. We thus find that when the system is in the superconducting phase, the current density in the London limit has the form (5.1), with $\Lambda_{T}$ varying from $\Lambda$ to $\infty$ as $T$ goes from 0 to $T_{c}$.

It is interesting to note that the Meissner effect occurs for any value of $\epsilon_{0} \neq 0$. If $\epsilon_{0}=0$ (for example if we let $V=0)$, then, for $T>0, \beta \epsilon_{0}=0$ and from $(5.27 \mathrm{~b})$ we see that $\Lambda / \Lambda_{T}=0$. The paramagnetic part of the current density is then

$$
\lim _{\mathbf{q} \rightarrow 0} \mathbf{j}_{P}(\mathbf{q})=\frac{n e^{2}}{m c} \mathfrak{a}(\mathbf{q}),
$$

and the total current in the $\mathbf{q} \rightarrow 0$ limit becomes

$$
\lim _{\mathbf{q} \rightarrow 0} \mathbf{j}(\mathbf{q})=0 \text {. }
$$

This is also true when $T=0$, though in this case one must be careful of the order in which the various limits are taken.

\section{Current Density}

We now evaluate the spacial distribution of the current density and exhibit it in a form similar to that proposed by Pippard. The method we use follows that 
of one of the authors ${ }^{7}$ who carried out a similar calculation for an energy-gap model.

Beginning with (5.15), setting $\mathbf{q}=\mathbf{k}^{\prime}-\mathbf{k}$, and using the fact that $\mathbf{q} \cdot \mathfrak{a}(\mathbf{q})=0$ and that we can write terms like $\mathbf{k} e^{i \mathbf{k} \cdot \mathbf{r}}$ as

$$
\mathbf{k} e^{i \mathbf{k} \cdot \mathbf{r}}=-i \boldsymbol{\nabla} e^{i \mathbf{k} \cdot \mathbf{r}},
$$

we can reduce all of the angular integrations to integrals of the form

$$
\int_{0}^{\pi} \sin \theta d \theta e^{ \pm i \mathbf{k} \cdot\left(\mathbf{r}-\mathbf{r}^{\prime}\right)}=\frac{2}{k R} \sin k R,
$$

where $R=\left|\mathbf{r}-\mathbf{r}^{\prime}\right|$. Then, using the relation

$$
\boldsymbol{\nabla}\left(\frac{\sin k R}{k R}\right)=\frac{k R \cos k R-\sin k R}{k R^{2}} \nabla R
$$

we finally obtain

$$
\mathbf{j}_{P}(\mathbf{r})=\frac{e^{2} \hbar^{2}}{2 m^{2} c \pi^{4}} \int d \mathbf{r}^{\prime} \frac{\left(\mathbf{A}\left(\mathbf{r}^{\prime}\right) \cdot \nabla^{\prime} R\right) \nabla R G_{s}(R)}{R^{4}}
$$

where

$$
G_{s}(R)=\int_{0}^{\infty} d k \int_{0}^{\infty} d k^{\prime} k k^{\prime} f(k, R) f\left(k^{\prime}, R\right) L\left(\epsilon, \epsilon^{\prime}\right)
$$

and

$$
f(k, R)=k R \cos k R-\sin k R .
$$

These equations correspond to (21.4)-(21.6) of reference 7. The entire current density $\mathbf{j}(\mathbf{r})$ can now be written

$$
\mathbf{j}(\mathbf{r})=\mathbf{j}_{P}(\mathbf{r})-\frac{n e^{2}}{m c} \mathbf{A}(\mathbf{r}) \text {. }
$$

It is convenient to subtract and add $G_{n}(R)$ inside the integral (5.33), where $G_{n}(R)$ is $G_{s}(R)$ evaluated at $\epsilon_{0}=0$. The integral evaluated with $G_{n}(R)$ gives the paramagnetic current contribution of a free electron gas and just cancels the term $-\left(n e^{2} / m c\right) \mathbf{A}(\mathbf{r})$, leaving the small Landau diamagnetic term in which we are not interested. We have left the interesting part of the current density:

$\mathbf{j}(\mathbf{r})=\frac{e^{2} \hbar^{2}}{2 m^{2} c \pi^{4}} \int d \mathbf{r}^{\prime} \frac{\left[G_{s}(R)-G_{n}(R)\right] \mathbf{R}\left[\mathbf{A}\left(\mathbf{r}^{\prime}\right) \cdot \mathbf{R}\right]}{R^{6}}$.

An inspection of $G_{s}(R),(5.34)$, reveals that the major contribution to the integral comes from the region $k, k^{\prime} \simeq k_{F}$ (i.e., very close to the Fermi surface). Since we are interested in values of $R \gtrsim$ penetration depth which is $\simeq 10^{-5} \mathrm{~cm}$, in the region of the major contribution, $k R \simeq k^{\prime} R \gg 1$. With this in mind, the product $f(k, R) f\left(k^{\prime} R\right)$ becomes

$$
\begin{array}{r}
f(k, R) f\left(k^{\prime}, R\right) \simeq k k^{\prime} R^{2} \cos k R \cos k^{\prime} R \\
=\frac{1}{2} k k^{\prime} R^{2}\left[\cos \left(k+k^{\prime}\right) R+\cos \left(k-k^{\prime}\right) R\right] \\
\simeq \frac{1}{2} k k^{\prime} R^{2} \cos \left(k-k^{\prime}\right) R,
\end{array}
$$

as $\cos \left(k+k^{\prime}\right) R$ is very rapidly oscillating in the region of interest.

Now, making the change of variable $d k=(d k / d \mathscr{E}) d \epsilon$, approximating the slowly varying terms $k+k^{\prime} \simeq 2 k_{F}$ and $d k / d \mathscr{E} \simeq 1 / \hbar v_{0}$, where $v_{0}$ is the velocity of an electron at the Fermi surface, and using the rapid convergence of the integral to extend large finite limits to infinite ones, we obtain

$G_{s}(R)-G_{n}(R)$
$\quad=-\frac{1}{2} k_{F}{ }^{4} R^{2}\left(\frac{d k}{d \mathscr{E}}\right)_{F}^{2} \pi^{2} \epsilon_{0}(0) \frac{\Lambda}{\Lambda_{T}} J(R, T)$,

where

and

$$
J(R, T)=I(R, 0)-I\left(R, \epsilon_{0}\right)
$$

$$
\begin{aligned}
I\left(R, \epsilon_{0}\right)=\frac{\Lambda_{T}}{\Lambda \pi^{2} \epsilon_{0}(0)} \int_{-\infty}^{+\infty} \int_{-\infty} d \epsilon d \epsilon^{\prime} L\left(\epsilon, \epsilon^{\prime}\right) & \\
& \times \cos \left[\left(\epsilon-\epsilon^{\prime}\right) \frac{R}{\hbar v_{0}}\right] .
\end{aligned}
$$

The current density then becomes

$$
\mathbf{j}(\mathbf{r})=\frac{-3}{4 \pi c \Lambda_{T}} \frac{\pi \epsilon_{0}(0)}{\hbar v_{0}} \int d \mathbf{r}^{\prime} \frac{J(R, T) \mathbf{R}\left[\mathbf{A}\left(\mathbf{r}^{\prime}\right) \cdot \mathbf{R}\right]}{R^{4}},
$$

where $\Lambda_{T}$ has been given by (5.25).

The current density has now been written in a form in which it is easily comparable to Eq. (1.3), proposed by Pippard for a pure superconductor, where we identify $1 / \xi_{0}$ with the microscopic quantities:

$$
\frac{1}{\xi_{0}}=\frac{\pi \epsilon_{0}(0)}{\hbar v_{0}}
$$

and where $J(R, T)$ is to be compared with the exponential function, $\exp \left[-R / \xi_{0}\right]$. We have defined $J(R, T)$ so that it has the same integral as $\exp \left[-R / \xi_{0}\right]$ :

$$
\int_{0}^{\infty} J(R, T) d R=\xi_{0} .
$$

As evaluated in Appendix $\mathrm{C}, J(R, T)$ is given by

$$
\begin{aligned}
J(R, T) & =\frac{\Lambda_{T}}{\Lambda} \frac{\epsilon_{0}(T)}{\epsilon_{0}(0)}\left[\tanh \frac{\epsilon_{0}(T)}{2 k T}\right. \\
& \left.-\frac{2 \epsilon_{0}(T)}{\pi} \int_{0}^{\infty} d \epsilon^{\prime} \sin \left(\frac{2 R}{\hbar v_{0}} \epsilon^{\prime}\right) \frac{1-2 f\left(E^{\prime}\right)}{\epsilon^{\prime} E^{\prime}}\right]
\end{aligned}
$$

from which (5.44) can be established. In the London limit, where $\mathbf{A}(\mathbf{r})$ varies so slowly compared to the coherence distance $\xi_{0}$ that it may be taken out of the integral sign, we obtain

$$
\mathbf{j}(\mathbf{r})=-\frac{1}{c \Lambda_{T}} \mathbf{A}(\mathbf{r}),
$$

as has been shown previously. 


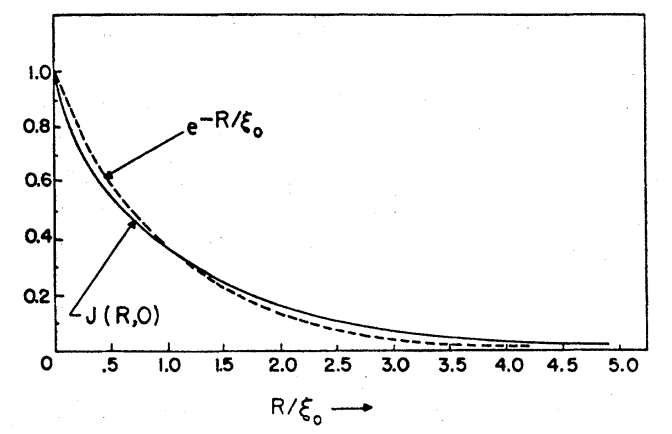

Fig. 5. The kernel $J(R, 0)$ for the current density at $T=0^{\circ} \mathrm{K}$ vs $R / \xi_{0}$, compared with the Pippard kernel, $\exp \left(-R / \xi_{0}\right)$.

With this normalization of $J(R, T)$ it turns out that most of the temperature variation of the current density is contained in $\Lambda_{T}$, and that the integral does not produce effects that vary very much with the temperature. This will be made clear later in the calculation of the penetration depth as a function of the temperature.

At $T=0, J(R, 0)$ has the simple form

$$
J(R, 0)=\frac{2}{\pi} \int_{2 \epsilon_{0}(0) R / \hbar v_{0}}^{\infty} K_{0}(y) d y,
$$

and when $R=0$

$$
J(0,0)=1 .
$$

Thus $J(R, 0)$ not only has the same integral as the exponential but also the same value at $R=0$. A comparison of the two given in Fig. 5 shows that they are quite similar.

We may express $\xi_{0}$ in a form similar to that suggested by Faber and Pippard ${ }^{25}$ :

$$
\xi_{0}=a\left(\hbar v_{0} / k T_{c}\right),
$$

where $a$ was adjusted empirically from observed penetration depths. From (5.43), we find

$$
\xi_{0}=\frac{\hbar v_{0}}{\pi \epsilon_{0}(0)}=\frac{1}{\pi \epsilon_{0}(0)} \frac{k T_{c}}{k T_{c}}=0.18 \frac{\hbar v_{0}}{k T_{c}} .
$$

Our theoretical value of 0.18 is between the empirical estimates of 0.15 by Faber and Pippard ${ }^{25}$ and of 0.27 by Glover and Tinkham. ${ }^{27}$ Thus at the absolute zero our theory gives a current density very much like that proposed by Pippard. Since $J(R, T)$ varies slowly with $T$, most of the temperature variation is contained in the constant $\Lambda_{T}$.

\section{Penetration Depths}

A most important application of the equations we have derived for the current density is to the calculation of field penetration at a plane surface. The results depend to some extent on the boundary conditions for scattering of electrons at the surface. Pippard ${ }^{26}$ has given general solutions for the limiting cases for specular reflection and for random scattering, based on corresponding expressions derived by Reuter and Sondheimer $^{37}$ for the anomalous skin effect. The penetration depth, defined by

is given by

$$
\lambda=\frac{1}{H(0)} \int_{0}^{\infty} H(x) d x
$$

$$
\lambda=\frac{2}{\pi} \int_{0}^{\infty} \frac{d q}{q^{2}+K(q)},
$$

for specular reflection and by

$$
\lambda=\frac{\pi}{\int_{0}^{\infty} \ln \left[1+q^{-2} K(q)\right]},
$$

for random scattering, where $K(q)$ is defined by (5.3).

Limiting expressions have been given for $\xi_{0} / \lambda$ large or small compared with unity. The London limit corresponds to $\xi_{0} \ll \lambda$, in which case $K(q)$ is a constant $=4 \pi / \Lambda_{T} c^{2}$ over the important range of integration. The penetration depth is then

$$
\lambda_{L}(T)=\left(4 \pi / \Lambda_{T} c^{2}\right)^{\frac{1}{2}}
$$

For a free-electron gas, this reduces to the London value $\left(m c^{2} / 4 \pi n e^{2}\right)$ at $T=0^{\circ} \mathrm{K}$. Since, for most metals, $\xi_{0} \simeq 10^{-4} \mathrm{~cm}$ and $\lambda \simeq 5 \times 10^{-6} \mathrm{~cm}$, it is the opposite limit, $\xi_{0} \gg \lambda$, which is more applicable (except possibly near $\left.T_{c}\right)$. In this limit $J(R, T)$ does not vary much over the penetration depth, so that it is only the value at $R=0, J(0, T)$, which enters. Pippard ${ }^{25}$ has given expressions for $\lambda$ in this limit, called $\lambda_{\infty}$. For random scattering,

$$
\lambda_{\infty}=\frac{3^{1 / 6}}{(2 \pi)^{\frac{1}{3}}}\left(\frac{\xi_{0} \lambda_{L}{ }^{2}}{J(0, T)}\right)^{\frac{1}{3}},
$$

while the value for specular reflection is smaller by a factor $8 / 9$.

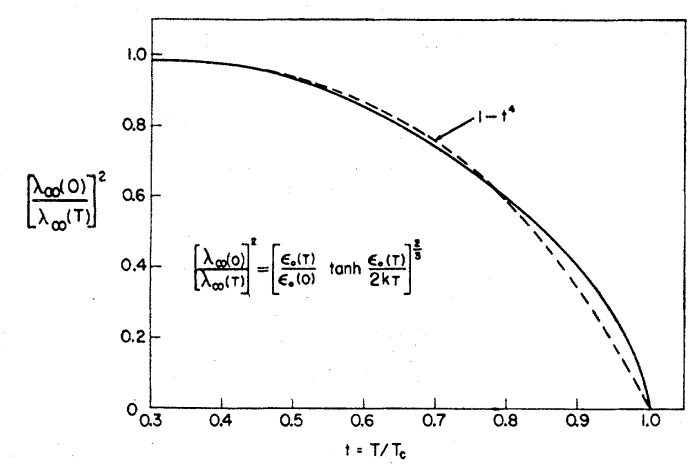

Fig. 6. The temperature variation of the penetration depth, $\lambda_{\infty}$, in the infinite coherence distance limit, $\left(\xi_{0} / \lambda\right) \rightarrow \infty$, compared with the empirical law, $[\lambda(0) / \lambda(T)]^{2}=1-t^{4}$.

${ }^{37}$ G. E. H. Reuter and E. H. Sondheimer, Proc. Roy. Soc. (London) A195, 336 (1948). 
THEORY OF SUPERCONDUCTIVITY

TABLE IV. Penetration depth, $\lambda$, at $T=0^{\circ} \mathrm{K} .{ }^{\mathrm{a}}$

\begin{tabular}{|c|c|c|c|c|c|c|c|c|c|}
\hline \multirow[b]{2}{*}{ Metal } & \multirow{2}{*}{$\begin{array}{c}(1) \\
10^{6} \lambda_{L} \\
\mathrm{~cm}\end{array}$} & \multirow{2}{*}{$\begin{array}{c}(2) \\
10^{1} \hbar v_{0} / k T_{c} \\
\mathrm{~cm}\end{array}$} & \multirow{2}{*}{$\begin{array}{c}(3) \\
10^{4} \xi_{0} \\
\mathrm{~cm}\end{array}$} & \multirow{2}{*}{$\begin{array}{c}(4) \\
\xi_{0} / \lambda_{L}\end{array}$} & (5) & (6) & (7) & (8) & \multirow{2}{*}{$\begin{array}{l}\text { (9) } \\
\text { Obs. } \\
10^{6} \lambda \\
\mathrm{cm}\end{array}$} \\
\hline & & & & & Specular reflection & $\begin{array}{r}\text { ction } \\
10^{\circ} \lambda\end{array}$ & \multicolumn{2}{|c|}{ Random scattering } & \\
\hline $\begin{array}{l}\text { Tin } \\
\text { Aluminum }\end{array}$ & $\begin{array}{l}3.5 \\
1.6\end{array}$ & $\begin{array}{l}1.4 \\
8.2\end{array}$ & $\begin{array}{l}0.25 \\
1.5\end{array}$ & $\begin{array}{l}7.3 \\
93 .\end{array}$ & $\begin{array}{l}1.4 \\
2.8\end{array}$ & $\begin{array}{l}4.8 \\
4.4\end{array}$ & $\begin{array}{l}1.6 \\
3.2\end{array}$ & $\begin{array}{l}5.7 \\
5.2\end{array}$ & $\begin{array}{l}5.1 \\
4.9\end{array}$ \\
\hline
\end{tabular}

a Values of $\lambda_{L}$ and $v_{0}$ are those estimated by Faber and Pippard ${ }^{25}$ from high-frequency skin resistance and normal electronic specific heat. The value of $\xi_{0}$ is $0.18 h v_{0} / k T_{c}$, as in (5.43). Ratios in columns 5 and 7 are taken from Fig. 6 . Observed values are from reference 25 .

The temperature dependence of $\lambda_{\infty}$ can be obtained directly from (5.45). The second term vanishes when $R=0$, so that we have

$$
\frac{\lambda_{\infty}{ }^{2}(0)}{\lambda_{\infty}{ }^{2}(T)}=\left[\frac{\epsilon_{0}(T) \tanh \left[\frac{1}{2} \beta \epsilon_{0}(T)\right]}{\epsilon_{0}(0)}\right]^{\frac{2}{3}} .
$$

A plot of this quantity on a reduced temperature scale is given in Fig. 6. It is plotted in this way so that a comparison can be made with the empirical law:

$$
\lambda^{2}(0) / \lambda^{2}(t)=1-t^{4}
$$

based on the Gorter-Casimir two-fluid model. It is seen that our theory is very close to the empirical law except for temperatures very close to $T_{c}$. It is in this region that the approximation $\xi_{0} \gg \lambda$ becomes invalid as a result of $\lambda$ increasing with $T$. The corrections are such as to reduce the theoretical values so as to bring them closer to the experimental values.

To determine $\lambda(T)$ for intermediate cases, we plot in Fig. 7 the quantity $\lambda(T) / \lambda_{L}(T)$ as a function of $\xi_{0} /$ $\lambda_{L}(T)$. The calculations are based on use of the asymtotic forms for $K(q)$ near $T=0^{\circ}$ and $T=T_{c}$ given in Appendix C, and numerical integrations of (5.52) and (5.53). The curves for the two limiting temperatures are quite close together, indicating that the effect of the variation of $J(R, T)$ with temperature is rather small. This procedure is analogous to that of Pippard ${ }^{5}$ who gave a plot by means of which one can determine $\lambda$ from known values of $\xi_{0}$ and $\lambda_{L}$ for the case $J(R, 0)$ $=\exp \left(-R / \xi_{0}\right)$.

Using our theoretical expression (5.50) for $\xi_{0}$ and empirically estimated values of $v_{0}$ and $\lambda_{L}(0)$ for tin and aluminum, ${ }^{25}$ we have obtained $\xi_{0} / \lambda_{L}(0)$, and, using Fig. 7, have determined $\lambda(0) / \lambda_{L}(0)$ for these two metals (Table IV). The agreement between theory and experiment is reasonably good, the experimental values falling between the theoretical values calculated for random scattering and specular reflection.

The theory we have presented applies to a pure metal. Pippard ${ }^{5}$ has shown experimentally that the existence of a finite mean free path, $l$, due to impurity scattering, has the effect of increasing the penetration depth, and has suggested that it may be taken into account by introducing an extra factor, $\exp (-R / l)$, into the kernel for the current density. One of the authors ${ }^{7}$ has shown why such a factor may be expected from a theory of the diamagnetic properties based on an energy-gap model. Similar considerations apply to the theory developed in this section; however, effects of coherence on the scattering matrix elements introduce complications and the proper correction factor has not yet been worked out.

To complete the electrodynamics we should give a relation corresponding to the second London equation, the one which gives the time-rate of change of current when an electric field is present. Such a theory would require a calculation, not yet completed, of transport properties with our excited-state wave functions. We expect to find something similar to a two-fluid model, in which thermally excited electrons correspond to the normal component of the fluid. For frequencies such that $h \nu \ll \epsilon_{0}$, one may determine $\partial \mathbf{j} / \partial t$ for the superconducting component by taking the time derivative of the integral relation (5.2) and then setting $\partial \mathbf{A} / \partial t$ $=-c \mathbf{E}$ in the result (see reference 7 ).

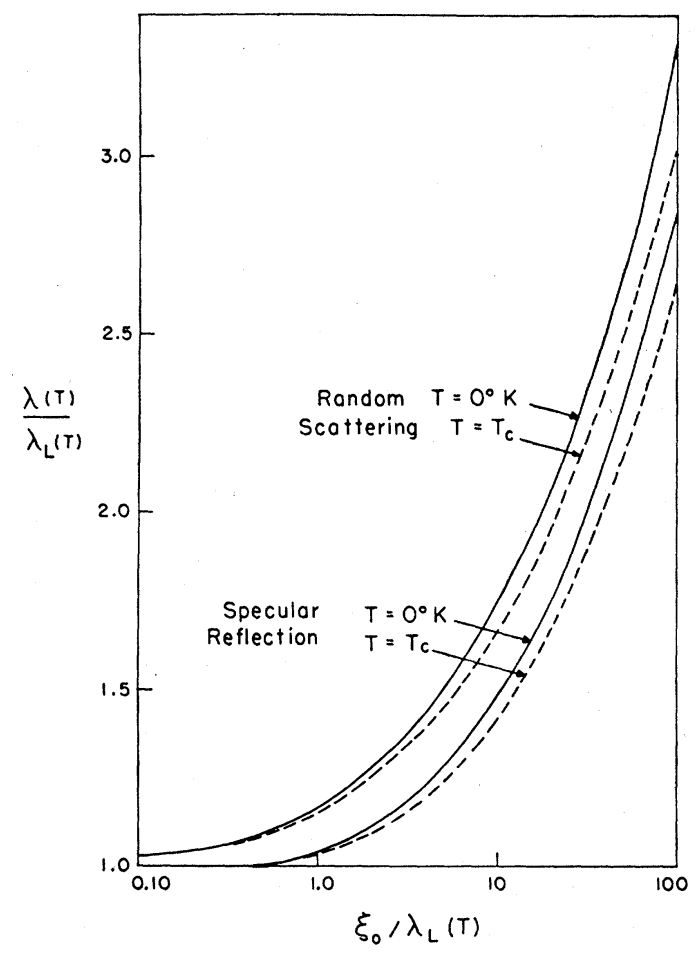

FIG. 7. The ratio $\lambda(T) / \lambda_{L}(T)$ vs $\xi_{0} / \lambda_{L}(T)$ for the boundary conditions of random scattering and specular reflection and for temperatures near $T=0^{\circ} \mathrm{K}$ and near $T=T_{c}$. The temperature variation of $\lambda_{L}(T)$ is given by $\lambda_{L}(T)=\left(\Lambda_{T} c^{2} / 4 \pi\right)^{2}$. 


\section{CONCLUSION}

Although our calculations are based on a rather idealized model, they give a reasonably good account of the equilibrium properties of superconductors. When the parameters of the theory are determined empirically, we find that we get agreement with observed specific heats and penetration depths to within the order of $10 \%$. Only the critical temperature involves the superconducting phase; the other two parameters required (density of states and average velocity at the Fermi surface) are determined from the normal phase. This quantitative agreement, as well as the fact that we can account for the main features of superconductivity is convincing evidence that our model is essentially correct.

The basis for the theory is a net attractive interaction between electrons for transitions in which the energy difference between the electron states involved is less than the phonon energy, $\hbar \omega$. For simplicity we have assumed a constant matrix element, $-V$, for transitions within an average energy $\hbar \omega$ of the Fermi surface and have neglected the repulsive interaction outside this region. In more accurate calculations one should take an interaction region dependent on the initial states of the electron and the transition involved, and also take into account any anisotropy in the Fermi surface and in the matrix elements. The fact that there is a law of corresponding states is empirical evidence that such effects are not of great importance. Neglect of the repulsive part of the interaction is in the spirit of the Bloch approximation for normal metals, and appears to be well justified in first approximation. Our theory may be regarded as an extension of the Bloch theory to superconductors in which we introduce only those interactions responsible for the transition.

An improvement in the general formulation of the theory is desirable. We have used that of Bardeen and Pines in which screening of the Coulomb field is taken into account by the Bohm-Pines collective model, and the phonon interaction between electrons is determined only to second order. Diagonal or self-energy terms in the net interaction have been omitted with the assumption that they are included in the Bloch energies of the normal state. When the phonon interaction is so large as to give superconductivity, higher order terms than the second may well be important. One should really have used a renormalized interaction in which such higher order terms are taken into account as well as possible. Very likely the assumption of two-particle interactions is a reasonably good one, so that the only effect would be a redefinition of the interaction constant $V$ in terms of microscopic quantities.

The discussion of the matrix elements in Sec. IV should be a good starting point for calculation of transport properties in the superconducting phase. Our excited state many-particle wave functions are not much more difficult to use in such calculations than the determinantal wave functions of the Bloch theory.

For calculation of boundary energies and related problems, one would like to introduce an order parameter which can decrease continuously from an equilibrium value for the superconducting phase to zero in the normal phase as the boundary is crossed. The Ginsburg-Landau theory and its extensions ${ }^{7}$ appear to give a good phenomenological description of such effects. Perhaps the energy gap, $2 \epsilon_{0}$, or, what is equivalent, the coherence distance, $\xi_{0}$, could be used for such a parameter.

Another problem, not yet solved, is the calculation of the paramagnetic susceptibility of the electrons in a superconductor, such as is required to account for Reif's data ${ }^{38}$ on the Knight shift in the nuclear paramagnetic resonance of colloidal mercury. Our ground state is for total spin $S=0$. It is possible that there is no energy gap between this state and those for $S \neq 0$. While a finite energy is required to turn over an individual spin, it might be possible to construct states analogous to those used in spin-wave theory in which each virtual pair has a small net spin, and for which the energy varies continuously with $S$. The explanation of the observed electronic paramagnetism (about twothirds that of the normal metal) would then be similar to that suggested by Reif himself.

In view of its success with equilibrium properties, it may be hoped that our theory will be able to account for these and for other so far unsolved problems.

The authors are indebted to many of their associates for discussions which have helped to clarify the problems involved. We should like to mention particularly discussions with C. P. Slichter and L. C. Hebel on calculation of matrix elements, with $\mathrm{D}$. Pines on the criterion for superconductivity, and with $\mathrm{K}$. A. Brueckner on the exactness of the solution for the ground state.

\section{APPENDIX A. CORRECTIONS TO GROUND STATE ENERGY}

We may estimate the accuracy of our superconducting ground state energy, $W_{0}$, measured relative to that of the normal state, by making a perturbation theory expansion, using the complete set of excited state superconducting wave functions as the basis functions of the expansion. We first consider the reduced, $H_{\text {red, }}$ which includes only pair transitions for pair momentum $q=0$, and then the effect of the neglected portion of the Hamiltonian, $H^{\prime}=H-H_{\text {red }}$.

To the second order, the ground state energy of $H_{\text {red }}$ is :

$$
\begin{aligned}
W_{0}= & \left(\Psi_{0}\left|H_{\text {red }}\right| \Psi_{0}\right)+\sum_{i \neq 0} \frac{\left|\left(\Psi_{i}\left|H_{\text {red }}\right| \Psi_{0}\right)\right|^{2}}{W_{0}-W_{i}}+\cdots \\
= & W_{0}+W_{0}{ }^{(2)}+\cdots .
\end{aligned}
$$

\footnotetext{
${ }^{38}$ F. Reif, Phys. Rev. 106, 208 (1957).
} 
Since the smallest excitation energy is $2 \epsilon_{0}$, the second order energy is overestimated by setting $\left|W_{0}-W_{i}\right|$ $=2 \epsilon_{0}$ and performing a closure sum. We obtain the inequality

$$
\begin{aligned}
W_{0}^{(2)} & \leq\left[\left(\Psi_{0}\left|H_{\mathrm{red}}{ }^{2}\right| \Psi_{0}\right)-\left(\Psi_{0}\left|H_{\mathrm{red}}\right| \Psi_{0}\right)^{2}\right] /\left(-2 \varepsilon_{0}\right) \\
& =\frac{w_{a}+w_{b}+w_{c}}{-2 \epsilon_{0}}
\end{aligned}
$$

where

$$
\begin{aligned}
w_{a}= & \left(\Psi_{0}\left|H_{0}{ }^{2}\right| \Psi_{0}\right)-\left(\Psi_{0}\left|H_{0}\right| \Psi_{0}\right)^{2}, \\
w_{b}= & \left(\Psi_{0}\left|H_{0} H_{v}+H_{v} H_{0}\right| \Psi_{0}\right) \\
& \quad-2\left(\Psi_{0}\left|H_{0}\right| \Psi_{0}\right)\left(\Psi_{0}\left|H_{v}\right| \Psi_{0}\right), \\
w_{c}= & \left(\Psi_{0}\left|H_{v}{ }^{2}\right| \Psi_{0}\right)-\left(\Psi_{0}\left|H_{v}\right| \Psi_{0}\right)^{2},
\end{aligned}
$$

and

$$
\begin{aligned}
& H_{0}=\sum_{k>k_{F}} \epsilon_{\mathrm{k}} b_{\mathrm{k}}{ }^{*} b_{\mathrm{k}}+\sum_{k<k_{F}}\left|\epsilon_{\mathrm{k}}\right| b_{\mathrm{k}} b_{\mathrm{k}}{ }^{*}, \\
& H_{v}=-V \sum_{\mathbf{k}, \mathrm{k}^{\prime}} b_{\mathbf{k}^{\prime}}{ }^{*} b_{\mathrm{k}},
\end{aligned}
$$

the sum in (A7) being carried out over the region $\left|\epsilon_{k}\right|$ and $\left|\epsilon_{k^{\prime}}\right|<\hbar \omega$. If terms in $W_{0}^{(2)}$ linear in the volume of the system are evaluated, the following expressions may be derived with the aid of the decomposition $(2.25)$ :

$$
\begin{array}{r}
w_{a}=8 N(0) \int_{0}^{\hbar \omega} d \epsilon h(\epsilon)[1-h(\epsilon)] \epsilon^{2}, \\
w_{b}=-16 N(0) V \int_{0}^{\hbar \omega} d \epsilon_{1} \int_{0}^{\hbar \omega} d \epsilon_{2}\left\{h\left(\epsilon_{1}\right)\left[1-h\left(\epsilon_{1}\right)\right]\right. \\
\left.\quad \times h\left(\epsilon_{2}\right)\left[1-h\left(\epsilon_{2}\right)\right]\right\}^{\frac{1}{2}}\left[1-2 h\left(\epsilon_{1}\right)\right] \epsilon_{1}, \\
w_{c}=8[N(0) V]^{2}\left[\int_{0}^{\hbar \omega} d \epsilon_{1} h\left(\epsilon_{1}\right)\left[1-h\left(\epsilon_{1}\right)\right]\right]^{2} \\
\quad \times \int_{0}^{\hbar \omega} d \epsilon_{2}\left[1-2 h\left(\epsilon_{2}\right)\right]^{2} .
\end{array}
$$

With the use of the relations (2.35), (2.36), and (2.37), terms linear in the volume in (A2) become

$$
\begin{aligned}
&-2 \varepsilon_{0} W_{0}(2) \leq 8 N(0) \int_{0}^{\hbar \omega} d \epsilon \epsilon^{2}\{h(\epsilon)[1-h(\epsilon)] \\
&\left.-\frac{\epsilon_{0}{ }^{2}}{2 E^{2}}+\frac{\epsilon_{0}{ }^{2}}{4 E^{2}}\right\}=0,
\end{aligned}
$$

and therefore $W_{0}^{(2)} / W_{0}$ vanishes in the limit of a large system.

It is likely that $\left(\Psi_{0}\left|H_{\text {red }}{ }^{n}\right| \Psi_{0}\right)-\left(\Psi_{0}\left|H_{\text {red }}\right| \Psi_{0}\right)^{n}$ also vanishes in this limit for $n$ small compared with the total number of valance electrons in the system. For $\Psi_{0}$ to be an exact eigenfunction of $H_{\text {red }}$, in the statistical limit, it is required that the above condition hold for all $n$. Since this requirement can be shown to hold in the strong coupling limit, it is possible that $\Psi_{0}$ is exact in the statistical limit for all values of the coupling constant, although no proof has been found at this time.

We may estimate the effect of the neglected portion of the Hamiltonian, $H^{\prime}=H-H_{\text {red }}$ by a similar perturbation calculation. To second order in $H^{\prime}$ the correction to the superconducting ground state energy $W_{0}$ (measured relative to the normal ground state) is

$$
W^{\prime}=\sum_{i} \frac{\left|\left(i\left|H^{\prime}\right| 0\right)\right|^{2}}{W_{0}-W_{i}},
$$

where

$$
\begin{aligned}
\left(i\left|H^{\prime}\right| 0\right) & =-V\left\{\left[h\left(\mathbf{k}_{0}\right)\left(1-h\left(\mathbf{k}_{0}{ }^{\prime}\right)\right) h(\mathbf{k})\left(1-h\left(\mathbf{k}^{\prime}\right)\right)\right]^{\frac{1}{3}}\right. \\
& \left.-\left[h\left(\mathbf{k}_{0}^{\prime}\right)\left(1-h\left(\mathbf{k}_{0}\right)\right) h\left(\mathbf{k}^{\prime}\right)(1-h(\mathbf{k}))\right]^{\frac{1}{3}}\right\} .
\end{aligned}
$$

The first term in $\left(i\left|H^{\prime}\right| 0\right)$ may be identified with breaking up a pair in $\mathbf{k}_{0}$, the spin-down member going to $-\mathbf{k}_{0}{ }^{\prime} \downarrow$ and breaking up a pair in $\mathbf{k}$, the spin-up member going into $\mathbf{k}^{\prime} \uparrow$. The second term arises from $\mathbf{k}_{0}{ }^{\prime}$ and $\mathbf{k}^{\prime}$ being occupied in the ground state and arriving at the same intermediate state by $\mathbf{k}_{0}{ }^{\prime} \uparrow \rightarrow \mathbf{k}_{0} \uparrow$ and $-\mathbf{k}^{\prime} \downarrow \rightarrow-\mathbf{k} \downarrow$. The transitions involving particles with parallel spin have been neglected since their contribution is reduced by exchange.

Since the distribution of particle changes differs only over an energy region several $\epsilon_{0}$ wide, it is to be expected that most of $W^{\prime}$ will cancel between the normal and superconducting phases. To estimate the energy difference, we shall carry out the sums over a region $6 \epsilon_{0}$ wide. Inserting typical values in (A12), we find

$$
W^{\prime} \sim-\frac{V^{2}\left[3 N(0) \epsilon_{0}\right]^{3}}{2 \epsilon_{0}}\left(\frac{3 \epsilon_{0}}{E_{F}}\right) \sim 10^{-3} W_{0},
$$

where the factor $\left(3 \epsilon_{0} / E_{F}\right)$ comes from the average reduction in phase space as a result of conservation of momentum of the pairs making transitions.

These estimates indicate that although the total energy associated with $H^{\prime}$ may be significant, the effect of $H^{\prime}$ on the condensation energy is very small. It should be possible to obtain any required quantitative corrections to $W_{0}$ by use of perturbation theory.

\section{APPENDIX B. CHANGE IN ZERO-POINT ENERGY OF LATTICE VIBRATIONS}

The contribution to the condensation energy from the change in zero-point energy of the lattice can be estimated on the basis of the Bardeen and Pines collective ion-electron treatment. ${ }^{16}$ Their theory gives

$$
\begin{aligned}
& W_{Z P^{(s)}}-W_{Z P}{ }^{(n)} \\
& =\sum_{\mathbf{k}, \mathbf{K}} \frac{\left|M_{\mathrm{k}}\right|^{2}\left\{\left(n_{\mathrm{k}}^{(s)}-n_{\mathrm{k}}{ }^{(n)}\right)-\left(n_{\mathrm{k}+\kappa}{ }^{(s)}-n_{\mathrm{k}+\boldsymbol{\kappa}}{ }^{(n)}\right)\right\}}{\epsilon_{\mathrm{k}}-\epsilon_{\mathrm{k}+\boldsymbol{\kappa}}-\hbar \omega},
\end{aligned}
$$

where $n_{\mathrm{k}}(s)$ is the average occupation number in the superconducting state and $n_{\mathrm{k}}{ }^{(n)}$ that for the normal 
state. The sum over $\boldsymbol{\kappa}$ ranges over the first zone and $\mathbf{k}+\mathbf{k}$ is to be interpreted as the corresponding reduced wave vector so that Umklapp processes may be taken into account. If $\mathbf{k}+\boldsymbol{k}$ is replaced by $\mathbf{k}$ and $\boldsymbol{k}$ by $-\boldsymbol{k}$ in the terms containing $n_{\mathrm{k}+\mathrm{k}}$, (B1) reduces to

$$
\begin{aligned}
& W_{Z P^{(s)}}-W_{Z P}{ }^{(n)} \\
& =\sum_{\mathbf{k}, \boldsymbol{k}} \frac{2\left|M_{\mathrm{k}}\right|^{2}\left(\epsilon_{\mathrm{k}}-\epsilon_{\mathrm{k}+\kappa}\right)\left(n_{\mathrm{k}}{ }^{(s)}-n_{\mathrm{k}}{ }^{(n)}\right)}{\left(\epsilon_{\mathrm{k}}-\epsilon_{\mathrm{k}+\kappa}\right)^{2}-\left(\hbar \omega_{\kappa}\right)^{2}} .
\end{aligned}
$$

If the sum over $\boldsymbol{\kappa}$ is carried out, it is seen that the quantity multiplying the difference in occupation numbers is almost independent of $\mathbf{k}$. Since $n_{\mathrm{k}}{ }^{(8)}-n_{\mathrm{k}}{ }^{(n)}$ is antisymmetric with respect to the Fermi surface and differs from zero only in a range of the order of several $\epsilon_{0}$, it follows that the change in zero-point energy will be small compared to $W_{\mathbf{0}}$.

A rough estimate of the sum gives

$$
\begin{aligned}
& W_{Z P^{(s)}}-W_{Z P} P^{(n)}=-2\left\langle\left|M_{\kappa}\right|^{2}\right\rangle \int_{0}^{\hbar \omega} N(0) h(\epsilon) d \epsilon \\
& \times \int_{\varepsilon_{F}}^{\varepsilon_{Z}} N\left(\epsilon^{\prime}\right)\left\{\frac{\epsilon-\epsilon^{\prime}}{\left(\epsilon-\epsilon^{\prime}\right)^{2}-(\hbar \omega)^{2}}+\frac{\epsilon+\epsilon^{\prime}}{\left(\epsilon+\epsilon^{\prime}\right)^{2}-(\hbar \omega)^{2}}\right\} d \epsilon^{\prime} \\
& \simeq-2\left\langle\left|M_{\kappa}\right|^{2}\right\rangle \int_{0}^{\hbar \omega} N(0) h(\epsilon) d \epsilon \cdot 2 \epsilon\left[N\left(\mathcal{E}_{Z}\right) / \mathcal{E}_{Z}\right] \\
& \simeq-2 \frac{\left\langle\left|M_{\kappa}\right|^{2}\right\rangle}{\mathcal{E}_{Z}}[N(0)]^{2} \epsilon_{0}^{2} \simeq\left(\hbar \omega / \mathscr{E}_{Z}\right) W_{0} \simeq 10^{-3} W_{0},
\end{aligned}
$$

where $\mathcal{E}_{Z}$ is the energy at the zone boundary and typical values have been inserted for the parameters. Thus, it appears that the lattice zero-point energy should have little effect on the condensation energy.

\section{APPENDIX C. EVALUATION OF THE KERNEL IN THE PIPPARD INTEGRAL}

According to (5.41), the kernel of the Pippard integral expression for the current density at any temperature $T$ may be written

$$
\begin{aligned}
& J(R, T)=I(R, 0)-I\left(R, \epsilon_{0}\right) \\
& =\frac{\Lambda_{T}}{\pi^{2} \epsilon_{0}(0) \Lambda} \int_{-\infty}^{+\infty}\left\{\frac{f\left(\epsilon^{\prime}\right)-f(\epsilon)}{\epsilon-\epsilon^{\prime}}-L\left(\epsilon, \epsilon^{\prime}\right)\right\} \cos \alpha\left(\epsilon-\epsilon^{\prime}\right) d \epsilon d \epsilon^{\prime},
\end{aligned}
$$

where $\alpha=R / \hbar v_{0}$. The limits should really be $\pm \hbar \omega$, but the convergence is sufficiently rapid in the weak coupling limit so that we may replace $\hbar \omega$ by $\infty$ without appreciable error. One integration can be performed if use is made of the symmetry of the integrand in $\epsilon$ and $\epsilon^{\prime}$. We shall use the second form given for $L\left(\epsilon, \epsilon^{\prime}\right)$ in (4.22), with the lower $(+)$ signs. From the symmetry for positive and negative values of $\epsilon$ and $\epsilon^{\prime}$, the integral may be written as the sum of two integrals with limits 0 and $\infty$ :

$$
J(R, T)=\left[\Lambda_{T} / \pi^{2} \epsilon_{0}(0) \Lambda\right]\left(I_{1}+I_{2}\right),
$$

where

$$
\begin{aligned}
& I_{1}=2 \int_{0}^{\infty} \int_{0}^{\infty} \frac{\left[F(\epsilon)-F\left(\epsilon^{\prime}\right)\right] \cos \alpha \epsilon \cos \alpha \epsilon^{\prime}}{\epsilon^{2}-\epsilon^{\prime 2}} d \epsilon d \epsilon^{\prime}, \\
& I_{2}=2 \int_{0}^{\infty} \int_{0}^{\infty} \frac{\left[G(\epsilon)-G\left(\epsilon^{\prime}\right)\right] \epsilon \epsilon^{\prime} \sin \alpha \epsilon \sin \alpha \epsilon^{\prime} d \epsilon d \epsilon^{\prime}}{\epsilon^{2}-\epsilon^{\prime 2}},
\end{aligned}
$$

and

$$
\begin{aligned}
& F(\epsilon)=[1-2 f(\epsilon)] \epsilon-[1-2 f(E)]\left(E+\epsilon_{0}{ }^{2} E^{-1}\right), \\
& G(\epsilon)=[1-2 f(\epsilon)] \epsilon^{-1}-[1-2 f(E)] E^{-1} .
\end{aligned}
$$

Unless the argument is given explicitly, $\epsilon_{0} \equiv \epsilon_{0}(T)$.

If integration over the region about $\epsilon^{\prime}=\epsilon$ is made by principal parts, each of the two terms in $I_{1}$ (and each of the two terms in $I_{2}$ ) will give equal contributions. Care must be taken to take the same limits for $\epsilon$ and $\epsilon^{\prime}$. Thus we may write

$$
I_{1}=4{P^{\prime}}_{a \rightarrow 0, b \rightarrow \infty} \int_{a}^{b} \int_{a}^{b} \frac{F(\epsilon) \cos \alpha \epsilon \cos \alpha \epsilon^{\prime} d \epsilon d \epsilon^{\prime}}{\epsilon^{2}-\epsilon^{\prime 2}} .
$$

Here $\mathcal{Q}^{\prime}$ indicates the principal part of the integral of $\epsilon^{\prime}$ past $\epsilon^{\prime}=\epsilon$ is to be taken. Since $F(\epsilon) \rightarrow 0$ as $\epsilon \rightarrow \infty$, the value of the integral does not depend on how the upper limits of the integrals over $\epsilon$ and $\epsilon^{\prime}$ are approached. Thus we may set $b=\infty$ for both, and integrate over $\epsilon^{\prime}$ first. This would not have been true if we had not included $I(R, 0)$, the normal state contribution, which is equivalent to the term proportional to $\mathbf{A}$ in the expression for the current density. The lower limit is more critical; we must take $a$ the same for both $\epsilon$ and $\epsilon^{\prime}$ and approach the limit $a=0$ only in the final result. The integral over $\epsilon^{\prime}$ may be obtained from

$$
\int_{a}^{\infty} \frac{\cos \alpha \epsilon^{\prime} d \epsilon^{\prime}}{\epsilon^{2}-\epsilon^{\prime 2}}=\int_{0}^{\infty} \frac{\cos \alpha \epsilon^{\prime} d \epsilon^{\prime}}{\epsilon^{2}-\epsilon^{\prime 2}}-\int_{0}^{a} \frac{d \epsilon^{\prime}}{\epsilon^{2}-\epsilon^{\prime 2}} .
$$

In the second integral on the right we have assumed that $a$ is sufficiently small so that $\cos \alpha \epsilon^{\prime}$ may be replaced by unity. The first integral on the right may be evaluated by contour integration and the second directly to give

$$
{\rho^{\prime}}^{\prime} \int_{a}^{\infty} \frac{\cos \alpha \epsilon^{\prime} d \epsilon^{\prime}}{\epsilon^{2}-\epsilon^{\prime 2}}=\frac{1}{2} \pi \frac{\sin \alpha \epsilon}{\epsilon}-\frac{1}{2 \epsilon} \ln \left(\frac{\epsilon+a}{\epsilon-a}\right) .
$$

Similarly, to evaluate $I_{2}$, we have

$$
\rho^{\prime} \int_{0}^{\infty} \frac{\epsilon^{\prime} \sin \alpha \epsilon^{\prime} d \epsilon^{\prime}}{\epsilon^{2}-\epsilon^{\prime 2}}=-\frac{1}{2} \pi \cos \alpha \epsilon .
$$


In this integral we may take $a=0$, since the integrand is not singular at the origin. Combining $I_{1}$ and $I_{2}$, we find

$$
\begin{aligned}
I_{1}+I_{2}=-2 \int_{a}^{\infty} F(\epsilon) \ln \left(\frac{\epsilon+a}{\epsilon-a}\right) \frac{d \epsilon}{\epsilon} \\
\quad+\pi \int_{0}^{\infty}\left[F(\epsilon)-\epsilon^{2} G(\epsilon)\right] \frac{\sin 2 \alpha \epsilon}{\epsilon} d \epsilon .
\end{aligned}
$$

In the limit $a \rightarrow 0$, the first integral gives a contribution only near $\epsilon=0$. We may therefore replace $F(\epsilon)$ by $F(0)$. The logarithmic integral may then be evaluated, and we find

$$
\begin{aligned}
& I_{1}+I_{2}=\pi^{2} \epsilon_{0}\left[1-2 f\left(\epsilon_{0}\right)\right] \\
& \quad-2 \pi \epsilon_{0}^{2} \int_{0}^{\infty} \frac{1-2 f(E)}{E} \frac{\sin 2 \alpha \epsilon}{\epsilon} d \epsilon .
\end{aligned}
$$

Note that this expression vanishes in the two limits $\epsilon_{0} \rightarrow 0$ and $\alpha$ (or $R$ ) $\rightarrow \infty$, as it should. The second term vanishes as $R \rightarrow 0$.

From (C2) and (C12), we may write

$$
\begin{aligned}
J(R, T)=\frac{2 \Lambda_{T} \epsilon_{0}^{2}}{\pi \epsilon_{0}(0) \Lambda} \int_{0}^{\infty}\left\{\frac{1-2 f\left(\epsilon_{0}\right)}{\epsilon_{0}}\right. & \\
& \left.\quad-\frac{1-2 f(E)}{E}\right\} \frac{\sin 2 \alpha \epsilon}{\epsilon} d \epsilon,
\end{aligned}
$$

which is equivalent to $(5.45)$ of the text.

Since $f=0$ when $T=0$, we can write

$$
J(R, O)=\frac{2 \epsilon_{0}(0)}{\pi}\left(\frac{\pi}{2 \epsilon_{0}(0)}-\int_{0}^{\infty} \frac{\sin 2 \alpha \epsilon}{\epsilon E} d \epsilon\right) .
$$

This can be put into a form convenient for evaluation by using

$$
\begin{aligned}
\frac{\partial}{\partial \alpha} \int_{0}^{\infty} \frac{\sin 2 \alpha \epsilon}{\epsilon E} d \epsilon & =2 \int_{0}^{\infty} \frac{\cos 2 \alpha \epsilon}{E} d \epsilon \\
& =2 K_{0}\left(2 \alpha \epsilon_{0}\right),
\end{aligned}
$$

where $K_{0}$ is a modified Bessel function ${ }^{39}$ which falls off exponentially for large values of its argument. If we now observe that $\int_{0}^{\infty} K_{0}(y) d y=\pi / 2$, we obtain

$$
J(R, O)=\frac{2}{\pi} \int_{2 R / \pi \xi_{0}}^{\infty} K_{0}(y) d y .
$$

We next consider the integral of $J(R, T)$ with respect to $R$. If we introduce a convergence factor, we may

${ }^{39}$ See Erdelyi, Magnus, Oberhettinger, and Tricomi, Higher
Transcendental Functions (McGraw Hill Book Company, Inc., New York, 1953), Vol. 2, pp. 5 and 19. integrate under the sign in (C13):

$$
\lim _{\gamma \rightarrow 0} \int_{0}^{\infty} e^{-\gamma R} \sin \left(2 \epsilon R / \hbar v_{0}\right) d R=\frac{1}{2} \hbar v_{0} / \epsilon .
$$

Thus, remembering that $\xi_{0}=h v_{0} /\left[\pi \epsilon_{0}(0)\right]$, we find

$$
\begin{aligned}
& \frac{1}{\xi_{0}} \int_{0}^{\infty} J(R, T) d R \\
& \quad=\frac{\epsilon_{0}^{2} \Lambda_{T}}{\Lambda} \int_{0}^{\infty}\left\{\frac{1-2 f\left(\epsilon_{0}\right)}{\epsilon_{0}}-\frac{1-2 f(E)}{E}\right\} \frac{d \epsilon}{\epsilon^{2}} .
\end{aligned}
$$

According to (5.44) of the text, $\Lambda_{T}$ is defined so that the expression on the left is equal to unity. If we integrate the right hand side by parts, we find that

$$
\frac{\Lambda}{\Lambda_{T}}=-\epsilon_{0}^{2} \int_{0}^{\infty} \frac{d}{d E}\left(\frac{1-2 f(E)}{E}\right) \frac{d \epsilon}{E}
$$

This integral may be expressed in terms of $\epsilon_{0}$ and its temperature derivative by differentiating the defining integral for $\epsilon_{0}$ with respect to $\beta=1 / k T$. By a change of variable, $\beta \epsilon=x, \beta \epsilon_{0}=y$, we may write

$$
\begin{aligned}
\frac{1}{N(0) V}=\int_{0}^{\hbar \omega} \frac{1-2 f(E)}{E} d \epsilon & \\
& =\int_{0}^{\beta \hbar \omega} \frac{1-2 f\left(\left(x^{2}+y^{2}\right)^{\frac{1}{2}}\right)}{\left(x^{2}+y^{2}\right)^{\frac{1}{2}}} d x .
\end{aligned}
$$

We now differentiate with respect to $\beta$, remembering that $y$ depends on $\beta$. In the weak-coupling limit $\left[\epsilon_{0} \ll \hbar \omega, f(\beta \hbar \omega) \simeq 0\right]$, we find:

$$
0=\frac{1}{\beta}+\frac{d\left(\beta \epsilon_{0}\right)}{d \beta} \cdot \frac{\epsilon_{0}}{\beta} \int_{0}^{\hbar \omega} \frac{d}{d E}\left(\frac{1-2 f(E)}{E}\right) \frac{d \epsilon}{E} .
$$

The integral converges sufficiently rapidly so that we may replace the upper limit by $\infty$. Comparing (C19) and (C21), we find that

$$
\frac{\Lambda_{T}}{\Lambda}=\frac{1}{\epsilon_{0}} \frac{d\left(\beta \epsilon_{0}\right)}{d \beta}
$$

as stated in (5.25).

Finally, we shall derive an approximate expression for the transform $K(q)$ valid when $q h v_{0}>\epsilon_{0}(0)$. First we express $K(q)$ as an integral of $J(R, T)$. The transform of $\mathbf{j}(\mathbf{r})$ may be written

$$
\begin{aligned}
\mathbf{j}(\mathbf{q}) & =-\frac{c}{4 \pi} K(q) \mathfrak{a}(\mathbf{q}) \\
& =-\frac{3}{4 \pi c \Lambda_{T} \xi_{0}} \int \frac{\mathbf{R}[\mathbf{R} \cdot \mathfrak{a}(\mathbf{q})] e^{i \mathfrak{q} \cdot \mathbf{R}}}{R^{4}} J(R, T) d \tau .
\end{aligned}
$$


To carry out the integration over angles, we take the polar axis in the direction of $\mathbf{q}$ and set $u=\cos \theta$. This gives

$$
K(q)=\frac{3 \pi}{c^{2} \Lambda_{T} \xi_{0}} \int_{0}^{\infty} \int_{-1}^{+1}\left(1-u^{2}\right) e^{i R q u} J(R, T) d u d R .
$$

When (C13) is substituted for $J(R, T)$, we require the following integral:

$$
\begin{aligned}
g(b) & =\int_{0}^{\infty} \int_{-1}^{+1} e^{i R q u}\left(1-u^{2}\right) \sin \left(\epsilon R q / \epsilon_{1}\right) d u d R \\
& =\left(1-b^{2}\right) \ln \left(\frac{1+b}{1-b}\right)+2 b,
\end{aligned}
$$

where $\epsilon_{1}=\frac{1}{2} q \hbar v_{0}$ and $b=\epsilon / \epsilon_{1}$. We thus find

$K(q)=\frac{6 \pi \epsilon_{0}^{2}}{q c^{2} \Lambda \hbar v_{0}} \int_{0}^{\infty}\left\{\frac{1-2 f\left(\epsilon_{0}\right)}{\epsilon_{0}}-\frac{1-2 f(E)}{E}\right\} g(b) \frac{d \epsilon}{\epsilon}$.

Expansions of $g(b)$ for $b$ small and $b$ large are

$$
\begin{aligned}
& b<1: g(b)=4\left(b-\frac{1}{3} b^{3}-\frac{1}{15} b^{5} \cdots\right), \\
& b>1: g(b)=4\left(\frac{1}{3 b}+\frac{1}{15 b^{3}}+\cdots\right) .
\end{aligned}
$$

Further,

$$
\int_{0}^{\infty} g(b) \frac{d b}{b}=\frac{1}{2} \pi^{2}
$$

We may change the variable of integration in (C26) from $\epsilon$ to $b$, and then use different expansions for $b<1$ and $b>1$. One of the integrals required is

$$
\begin{aligned}
4 \int_{0}^{1} \frac{1-2 f(E)}{E} & \left\{b-\frac{1}{3} b^{3}-\frac{1}{15} b^{5}-\cdots\right\} \frac{d b}{b} \\
+4 & \int_{1}^{\infty} \frac{1-2 f(E)}{E}\left\{\frac{1}{3 b}+\frac{1}{15 b^{3}}+\cdots\right\} \frac{d b}{b} .
\end{aligned}
$$

In terms of $b$,

$$
E=\epsilon_{1}\left(b^{2}+b_{0}^{2}\right)^{\frac{1}{2}},
$$

where

$$
b_{0}=\epsilon_{0} / \epsilon_{1} .
$$

An approximate expression valid in the limit $b_{0} \ll 1$ may be obtained by neglecting $b_{0}$ in the integral for $b>1$, and also in terms in $b^{3}$ and higher in the integral for $b<1$. The only integral in which $b_{0}^{2}$ is not neglected in comparison with $b^{2}$ is the first term,

$$
4 \int_{0}^{1} \frac{1-2 f(E)}{E} d b=\frac{4}{\epsilon_{1}} \int_{0}^{\epsilon_{1}} \frac{1-2 f(E)}{E} d \epsilon
$$

which can be determined from (C20), the defining integral for $\epsilon_{0}$. For $\epsilon>\epsilon_{1}$ we may take $f(E)=0$. Thus we find

$$
\begin{aligned}
\frac{4}{\epsilon_{1}} \int_{0}^{\epsilon_{1}} \frac{1-2 f(E)}{E} d \epsilon \simeq \frac{4}{\epsilon_{1}}\left(\frac{1}{N(0) V}-\ln \frac{\hbar \omega}{\epsilon_{1}}\right) \\
=\frac{4}{\epsilon_{1}} \ln \frac{q \hbar v_{0}}{\epsilon_{0}(0)} .
\end{aligned}
$$

The latter form follows from the expression for $\epsilon_{0}(0)$ in the weak-coupling limit. The remaining terms cancel when $b_{0}^{2}$ is neglected:

$$
\begin{aligned}
\int_{0}^{1}\left\{\frac{b^{3}}{3}-\frac{b^{5}}{15}-\cdots\right. & \} \frac{d b}{b^{2}} \\
& +\int_{1}^{\infty}\left\{\frac{1}{3 b}+\frac{1}{15 b^{3}}+\cdots\right\} \frac{d b}{b^{2}}=0
\end{aligned}
$$

Thus we find that for $q h v_{0} / \epsilon_{0}(0)=\pi q \xi_{0} \gg 1$,

$$
K(q)=\frac{3 \pi^{2} \epsilon_{0}}{q c^{2} \Lambda \hbar v_{0}}\left\{1-2 f\left(\epsilon_{0}\right)-\frac{16 \epsilon_{0}}{\pi^{2} q \hbar v_{0}} \ln \left(\pi q \xi_{0}\right)\right\} .
$$

For $q$ very small, $K(q)$ approaches the constant value,

$$
K(q)=\lambda_{L}{ }^{-2}(T)=4 \pi /\left(\Lambda_{T} c^{2}\right), \quad(q \rightarrow 0)
$$

where $\Lambda_{T}$ is the temperature-dependent London constant defined by (C22). For intermediate values of $q$, one may interpolate between this constant value for $\pi q \xi_{0}<1$ and the asymptotic form (C36) for large $q$.

The two limiting cases are $T=0^{\circ} \mathrm{K}$ and $T$ close to $T_{c}$. At $T=0^{\circ} \mathrm{K}, \epsilon_{0} \rightarrow \epsilon_{0}(0)$ and $f\left(\epsilon_{0}\right) \rightarrow 0$, and we find

$K(q)=4 \pi /\left(\Lambda c^{2}\right)=1 / \lambda_{L}^{2}(0), \quad(q \rightarrow 0) ;$

$K(q)=\frac{3 \pi}{4 q \lambda_{L}{ }^{2}(0) \xi_{0}}\left\{1-\frac{16}{\pi^{3} q \xi_{0}} \ln \left(\pi q \xi_{0}\right)\right\}, \quad($ large $q)$.

Near $T_{c}$, one may determine $\Lambda_{T}$ from (C22) and (3.49):

$$
\Lambda / \Lambda_{T}=0.23 \beta_{c}{ }^{2} \epsilon_{0}{ }^{2}(T)=2(1-t),
$$

where $\beta_{c}=1 / k T_{c}$ and $t=T / T_{c}$. When $\epsilon_{0}(T)$ is small,

$$
1-2 f\left(\epsilon_{0}\right)=\tanh \left(\frac{1}{2} \beta \epsilon_{0}\right) \rightarrow \frac{1}{2} \beta_{c} \epsilon_{0}(T) .
$$

Thus the limiting expressions for $K(q)$ as $T \rightarrow T_{c}$ are

$$
\begin{aligned}
& K(q)=\frac{1}{\lambda_{L^{2}}{ }^{2}(T)}=\frac{2(1-t)}{\lambda_{L^{2}}(0)}, \quad(q \rightarrow 0) ; \\
& K(q)=\frac{3.75 \pi}{4 q \lambda_{L}{ }^{2}(T) \xi_{0}}\left\{1-\frac{18.3}{\pi^{3} q \xi_{0}} \ln \left(\pi q \xi_{0}\right)\right\},(\text { large } q) .
\end{aligned}
$$

The close similarity of the expressions for the two 
limiting cases is evident. The reason for the similarity is that $J(R, T)$ varies slowly with $T$.

At temperatures very close to $T_{c}, \lambda$ becomes larger than $\xi_{0}$, so that $\lambda(T)$ approaches $\lambda_{L}(T)$. A comparison of penetration depths measured near $T_{c}$ to those measured at low temperatures would then be expected to give

$$
\frac{\lambda(0)}{\lambda(T)}=\frac{\lambda(0)}{\lambda_{L}(0)} \frac{\lambda_{L}(0)}{\lambda(T)}=\frac{\lambda(0)}{\lambda_{L}(0)} \sqrt{2}(1-t)^{\frac{1}{2}} .
$$

This is to be compared with the ratio $2(1-t)^{\frac{1}{2}}$ which the empirical law, $\left(1-t^{4}\right)^{\frac{1}{2}}$, gives as $t \rightarrow 1$.

\section{APPENDIX D. CORRELATION OF ELECTRONS OF OPPOSITE SPIN}

Insight into the coherent structure of our ground state wave function may be obtained by an investigation of the correlation function for electrons of opposite spin. For the normal metal (in the Bloch approximation), electrons of antiparallel spin are entirely uncorrelated while for electrons of parallel spin there is the exchange correlation.

The correlation function $\rho_{\sigma^{\prime} \sigma^{\prime \prime}}\left(\mathbf{r}^{\prime}, \mathbf{r}^{\prime \prime}\right)$ for an $n$-electron system is defined as

$$
\begin{aligned}
\rho_{\sigma^{\prime} \sigma^{\prime \prime}}\left(\mathbf{r}^{\prime}, \mathbf{r}^{\prime \prime}\right)= & \sum_{i, j=1}^{2 n} \int \cdots \int d \tau_{1} \cdots d \tau_{n} \Psi^{*}\left(\mathbf{r}_{1} \cdots r_{n}\right) \\
& \times \Psi\left(\mathbf{r}_{1} \cdots r_{n}\right) \delta_{\sigma^{\prime}}\left(\mathbf{r}_{i}-\mathbf{r}^{\prime}\right) \delta_{\sigma^{\prime \prime}}\left(\mathbf{r}_{j}-\mathbf{r}^{\prime \prime}\right) .
\end{aligned}
$$

This can be written in second quantized form, following the notation of Sec. V, as

$$
\begin{array}{r}
\rho_{\sigma^{\prime} \sigma^{\prime \prime}}\left(\mathbf{r}^{\prime}, \mathbf{r}^{\prime \prime}\right)=\left(\Psi_{0}\left|\psi_{\sigma^{\prime \prime}} *\left(\mathbf{r}^{\prime \prime}\right) \psi_{\sigma^{\prime}} *\left(\mathbf{r}^{\prime}\right) \psi_{\sigma^{\prime}}\left(\mathbf{r}^{\prime}\right) \psi_{\sigma^{\prime \prime}}\left(\mathbf{r}^{\prime \prime}\right)\right| \Psi_{0}\right) \\
=\sum_{\mathbf{k}_{1}, \mathbf{k}_{2}, \mathbf{k}_{3}, \mathbf{k}_{4}}\left(\Psi_{0}\left|c^{*}\left(\mathbf{k}_{1}, \sigma^{\prime \prime}\right) c^{*}\left(\mathbf{k}_{2}, \sigma^{\prime}\right) c\left(\mathbf{k}_{3}, \sigma^{\prime}\right) c\left(\mathbf{k}_{4}, \sigma^{\prime \prime}\right)\right| \Psi_{0}\right) \\
\times e^{i\left(\mathbf{k}_{4}-\mathbf{k}_{1}\right) \cdot \mathbf{r}^{\prime \prime}+i\left(\mathbf{k}_{3}-\mathbf{k}_{2}\right) \cdot \mathbf{r}^{\prime}}, \quad(\mathrm{D} 2)
\end{array}
$$

where $\Psi_{0}$ is our ground-state wave function.

Of particular interest is the correlation function for electrons of opposite spin. If we define

$$
\rho_{A}=\rho_{\uparrow \downarrow}+\rho_{\downarrow \uparrow},
$$

use the matrix elements obtained in Sec. IV, and set $\mathbf{r}=\mathbf{r}^{\prime}-\mathbf{r}^{\prime \prime}$, we get at the absolute zero of temperature

where

$$
\rho_{A}(r)=n\left[\frac{1}{2} n+P_{A}(r)\right]
$$

$$
P_{A}(r)=\frac{1}{2 n}\left(\frac{1}{2 \pi}\right)^{6} \iint_{\mathcal{R}} d \mathbf{k} d \mathbf{k}^{\prime}\left\{e^{i\left(\mathbf{k}^{\prime}-\mathbf{k}\right) \cdot \mathbf{r}} \frac{\epsilon_{0}{ }^{2}(0)}{E E^{\prime}}\right\} .
$$

The integration is over $R$, the region of interaction $(|\epsilon|<\hbar \omega)$, and $n$ is the number of electrons of both spin directions per unit volume. The terms in the bracket give the number of electrons of opposite spin per unit volume one would find a distance $r$ away from an electron of given spin direction. The first term is just the average density, while the second gives the effects of correlation.

The integral of $P_{A}(r)$ gives the number of electrons of opposite spin correlated with a given electron. This is

$$
\begin{aligned}
\int P_{A}(r) d \tau= & \frac{\epsilon_{0}^{2}(0)}{2 n}\left(\frac{1}{2 \pi}\right)^{3} \int_{\mathbb{R}} d k \frac{1}{\epsilon^{2}+\epsilon_{0}^{2}(0)} \\
& \left.\simeq \frac{\epsilon_{0}^{2}(0)}{2 n} \frac{1}{\pi^{2}} \frac{d k}{d \mathcal{E}}\right]_{F} k_{F}^{2} \int_{0}^{\hbar \omega} \frac{d \epsilon}{\epsilon^{2}+\epsilon_{0}^{2}(0)} \\
& \simeq \frac{3}{4} \frac{1}{k_{F} \xi_{0}}=\frac{\pi n_{c}}{2}
\end{aligned}
$$

where we have given slowly varying functions their value at the Fermi surface, and have used (5.43) and $n_{c}=N(0) \epsilon_{0}(0)$. Thus the number of electrons of given spin correlated to one of opposite spin is of the order of the ratio of the number of electrons in coherent pairs to the total number of electrons in the system.

The range of the spacial correlation may be determined by investigating $P_{A}(r)$. We must then evaluate

$$
I=\left(\frac{1}{2 \pi}\right)^{3} \int_{\mathbb{R}} d \mathbf{k} \frac{e^{i \mathbf{k} \cdot \mathbf{r}}}{E}=\frac{1}{2 \pi^{2} r} \int_{k_{F}-\delta}^{k F^{*}+\delta} \frac{k \sin k r d k}{\left[\epsilon^{2}+\epsilon_{0}{ }^{2}(0)\right]^{1}} .
$$

To evaluate this, we make use of the sharp maximum of the integral near $\epsilon=0$ and the fact that the sine function is rapidly oscillating in this region for values of $r$ of interest, $k_{F} r \gg 1$. We set (as in Sec. V)

$$
\left.k \simeq k_{F}+\frac{d k}{d \mathscr{E}}\right]_{F} \epsilon=k_{F}+\epsilon /\left(h v_{0}\right)
$$

and

$$
\sin \left(k_{F}+\frac{\epsilon}{h v_{0}}\right) r \simeq \sin k_{F} r \cos \left(\frac{\epsilon}{h v_{0}} r\right)
$$

give slowly varying functions their value at the Fermi surface, and drop terms antisymmetric in $\epsilon$. Then, setting $x=\epsilon / \epsilon_{0}$, we get

$$
I=\frac{k_{F}}{\pi^{2} r} \frac{1}{h v_{0}} \sin k_{F} r \int_{0}^{a} \frac{\cos \left(r x / \pi \xi_{0}\right) d x}{\left(x^{2}+1\right)^{\frac{1}{2}}}
$$

where $a=\hbar \omega / \epsilon_{0} \gg 1$. The integral may be expressed as

$$
\begin{aligned}
\int_{0}^{a} \frac{\cos \left(r / \pi \xi_{0}\right) x}{\left(x^{2}+1\right)^{\frac{1}{2}}} d x=K_{0}\left(\frac{r}{\pi \xi_{0}}\right) & \\
& \quad-\int_{a}^{\infty} \frac{\cos \left(r x / \pi \xi_{0}\right)}{\left(x^{2}+1\right)^{\frac{1}{2}}} d x
\end{aligned}
$$

where $K_{0}$ is a modified Bessel function. ${ }^{39}$ Since $a \gg 1$ except for very small values of $r,\left(x^{2}+1\right)^{\frac{1}{2}} \simeq x$ in the 
last integral. Then, setting $t=r x /\left(\pi \xi_{0}\right)$, we have

$$
I=\frac{k_{F}}{\pi^{2} r} \frac{1}{h v_{0}} \sin k_{F r}\left[K_{0}\left(\frac{r}{\pi \xi_{0}}\right)-\int_{a r / \pi \xi_{0}}^{\infty} \frac{\cos t}{t} d t\right] .
$$

Asymptotically

$$
-\int_{x}^{\infty} \frac{\cos t}{t} d t \sim \frac{\sin x}{x}, x \gg 1
$$

while $K_{0}(x)$ falls off exponentially. Thus the asymptotic behavior of $P_{A}(r)$ is given by

$$
P_{A}(r) \sim \frac{9 n \epsilon_{0}^{2}(0)}{2(\hbar \omega)^{2}} \frac{\sin ^{2} k_{F} r \sin ^{2}\left(\frac{\epsilon_{0}}{\hbar \omega} \frac{r}{\pi \xi_{0}}\right)}{\left(k_{F} r\right)^{4}} .
$$

Since $a=h \omega / \epsilon_{0} \gg 1$, the range of correlation is determined by the $K_{0}$ function which drops off rapidly when $r /\left(\pi \xi_{0}\right) \gtrsim 1$. Thus correlation distance is the order of $\pi \xi_{0} \simeq 10^{-4} \mathrm{~cm}$.

\title{
Residual Resistivity of Gold Alloys : Dependence on Periodic Table*
}

\author{
F. J. BLATT \\ Department of Physics and Astronomy, Michigan State University, East Lansing, Michigan
}

(Received August 12, 1957)

\begin{abstract}
The residual resistivities of dilute alloys of gold are calculated by the same method which was employed in a similar study of the residual resistivities of copper and silver alloys. As in the earlier work, the calculated results agree well with experiment with the exception of the alloys gold-copper and gold-silver. The application of the modification of the Friedel sum condition to other problems is suggested.
\end{abstract}

\section{INTRODUCTION}

$I^{\mathrm{N}}$ $\mathrm{N}$ a previous paper ${ }^{1}$ attention was directed to the fact that the residual resistivities of dilute noble metal alloys exhibited a very systematic dependence on the position which the solute occupies in the periodic table of the elements. The most pronounced dependence is on the valence of the solute: $\Delta \rho$, the residual resistivity per atomic percent solute, increases approximately as the square of the valence difference $Z$. Since we are concerned here with monovalent solvent metals, we have $Z=Z^{\prime}-1$ where $Z^{\prime}$ is the valence of the solute. In addition to this well-known $Z^{2}$ dependence, for which Mott ${ }^{2}$ and Friedel ${ }^{3}$ have provided satisfactory explanations, $\Delta \rho$, for a given $Z$, also depends systematically on the row, or period, of the periodic table to which the solute belongs. Solutes which occupy the silver row of the periodic table invariably give rise to smaller values of $\Delta \rho$ in every one of the noble metals than do solutes which belong to the copper row. In the notation of I, we can express this experimental observation by the following inequalities

$$
\frac{\mathrm{Cu}(\mathrm{Cu})}{\mathrm{Cu}(\mathrm{Ag})}>1 ; \quad \frac{\mathrm{Ag}(\mathrm{Cu})}{\mathrm{Ag}(\mathrm{Ag})}>1 ; \quad \frac{\mathrm{Au}(\mathrm{Cu})}{\mathrm{Au}(\mathrm{Ag})}>1 .
$$

* Supported in part by the Air Force Office of Scientific Research, Air Research and Development Command.

1 F. J. Blatt, Phys. Rev. 108, 285 (1957). Hereafter, we shall refer to this paper as I.

2 N. F. Mott, Proc. Cambridge Phil. Soc. 32, 281 (1936).

$3 \mathrm{~J}$. Friedel, Advances in Physics (Taylor and Francis, Ltd., London, 1954), Vol. 3, p. 446.
Calculations of $\Delta \rho$ based on a free electron model, such as those of Mott, Friedel and others ${ }^{4,5}$ differ in their numerical results but do all lead to the conclusion

$$
\frac{\mathrm{Cu}(\mathrm{Cu})}{\mathrm{Cu}(\mathrm{Ag})}=\frac{\mathrm{Ag}(\mathrm{Cu})}{\mathrm{Ag}(\mathrm{Ag})}=\frac{\mathrm{Au}(\mathrm{Cu})}{\mathrm{Au}(\mathrm{Ag})}=1 \text {. }
$$

Calculations of the residual resistivities of dilute alloys of copper and silver where found to be in good agreement with experimental results if the expansion of the lattice in the neighborhood of a solute atom was taken into account in a manner suggested by Harrison. ${ }^{6}$ In the present article we report results of similar calculations on dilute alloys of gold.

\section{CALCULATION AND RESULTS}

It was shown in I that the discrepancy between the observed values of the ratios $\mathrm{Cu}(\mathrm{Cu}) / \mathrm{Cu}(\mathrm{Ag})$ and $\mathrm{Ag}(\mathrm{Cu}) / \mathrm{Ag}(\mathrm{Ag})$ and those calculated in a free electron approximation could be obviated by a suitable modification of the Friedel sum condition. ${ }^{3}$ The Friedel sum rule states that

$$
(2 / \pi) \sum_{l}(2 l+1) \delta_{l}=N,
$$

where $\delta_{l}$ are the phase shifts evaluated for electrons at the Fermi surface and $N$ is the excess charge,

\footnotetext{
${ }^{4}$ F. J. Blatt, Phys. Rev. 99, 1708 (1955).

${ }^{5} \mathrm{P}$. de Faget de Casteljau and J. Friedel, J. phys. radium 17, 27 (1956).

${ }^{6}$ W. A. Harrison (to be published).
} 